\title{
Actuation of thin nematic elastomer sheets with controlled heterogeneity
}

\author{
Paul Plucinsky, Marius Lemm, and Kaushik Bhattacharya \\ California Institute of Technology, Pasadena, California 91125, USA
}

November 3, 2016

\begin{abstract}
Nematic elastomers and glasses deform spontaneously when subjected to temperature changes. This property can be exploited in the design of heterogeneously patterned thin sheets that deform into a non-trivial shape when heated or cooled. In this paper, we start from a variational formulation for the entropic elastic energy of liquid crystal elastomers and we derive from it an effective two-dimensional metric constraint, which links the deformation and the heterogeneous director field. Our main results show that satisfying the metric constraint is both necessary and sufficient for the deformation to be an approximate minimizer of the energy. We include several examples which show that the class of deformations satisfying the metric constraint is quite rich.
\end{abstract}

\section{Contents}

1 Introduction and main results . . . . . . . . . . . . . . . . . . . . . . . . . . . . . . . . 2

1.1 Model for nematic elastomers with controlled heterogeneity . . . . . . . . . . . . . . 2

1.2 The metric constraint and overview of results . . . . . . . . . . . . . . . . . . . . 3

1.3 Nonisometric origami constructions under the metric constraint . . . . . . . . . . . 6

1.4 On the optimality of nonisometric origami . . . . . . . . . . . . . . . . . . . . . . . . 7

1.5 Examples of pure bending actuation under the metric constraint . . . . . . . . . . 9

1.6 The metric constraint as a necessary condition for bending . . . . . . . . . . . . . . . 12

2 Approximating the two-dimensional deformations for idealized actuation . . . . . . . . . 14

3 Incompressibility and the energy of three dimensional constructions . . . . . . . . . . . . 25

4 Nonisometric origami and an opitimal scaling law . . . . . . . . . . . . . . . . . . . . . . 32

5 Compactness for bending configurations and the metric constraint . . . . . . . . . . . . . 37

Appendices . . . . . . . . . . . . . . . . . . . . . . . . . . . . . . . . . . . 41

A Some facts about the entropic energy . . . . . . . . . . . . . . . . . . . . . . . . . 41

B Proof of Proposition 1.10 . . . . . . . . . . . . . . . . . . . . . . . . . . . . . . . . . 44

C On nonisometric origami . . . . . . . . . . . . . . . . . . . . . . . . . 45

D Proof of Proposition 2.3 . . . . . . . . . . . . . . . . . . . . . . . . . . . . . . . . . 48

E Geometric rigidity and nematic elastomers . . . . . . . . . . . . . . . . . . . . . . . . 54 


\section{Introduction and main results}

\subsection{Model for nematic elastomers with controlled heterogeneity}

We consider a thin sheet of nematic liquid crystal elastomer of thickness $h \ll 1$. Initially, the sheet occupies a flat region in space,

$$
\Omega_{h}:=\omega \times(-h / 2, h / 2), \quad \omega \subset \mathbb{R}^{2},
$$

where $\omega$ is an open, connected and bounded Lipschitz domain, which we call the midplane of the sheet. We envision that the liquid crystal molecules within the elastomer sheet are programmed so that there average alignment can be described macroscopically by a director field $N_{0}^{h}: \Omega_{h} \rightarrow \mathbb{S}^{2}$ at the initial temperature $T_{0}$. Upon changing the temperature from $T_{0}$ to the final temperature $T_{f}$, the sheet will spontaneously deform by a deformation $Y^{h}: \Omega_{h} \rightarrow \mathbb{R}^{3}$ which we assume minimizes the entropic elastic energy

$$
\mathcal{I}_{N_{0}^{h}}^{h}\left(Y^{h}\right):=\int_{\Omega_{h}} W^{e}\left(\nabla Y^{h}, \frac{\left(\nabla Y^{h}\right) N_{0}^{h}}{\left|\left(\nabla Y^{h}\right) N_{0}^{h}\right|}, N_{0}^{h}\right) d x .
$$

(If $\left(\nabla Y^{h}\right) N_{0}^{h}=0$ on a set of positive measure, then we set $\mathcal{I}_{N_{0}^{h}}^{h}\left(Y^{h}\right)=\infty$.) Following Bladon et al. [11] (see also Warner and Terentjev [43]), we take the entropic elastic energy density $W^{e}$ : $\mathbb{R}^{3 \times 3} \times \mathbb{R}^{3} \times \mathbb{R}^{3} \rightarrow \mathbb{R} \cup\{+\infty\}$ as

$$
W^{e}\left(F, N, N_{0}\right):=\frac{\mu}{2} \begin{cases}\operatorname{Tr}\left(F^{T}\left(\ell_{N}^{f}\right)^{-1} F \ell_{N_{0}}^{0}\right)-3 & \text { if } \operatorname{det} F=1, N, N_{0} \in \mathbb{S}^{2} \\ +\infty & \text { otherwise. }\end{cases}
$$

Here, $\mu>0$ is the shear modulus, $F$ is the deformation gradient, and $F^{T}$ denotes the transpose matrix of $F$. Moreover, $\ell_{N_{0}}^{0}, \ell_{N}^{f} \in \mathbb{R}_{s y m}^{3 \times 3}$ are the step length tensors at the initial temperature $T_{0}$ and final temperature $T_{f}$ respectively. They are defined by

$$
\begin{aligned}
\ell_{N_{0}}^{0} & :=r_{0}^{-1 / 3}\left(I_{3 \times 3}+\left(r_{0}-1\right) N_{0} \otimes N_{0}\right), \\
\ell_{N}^{f} & :=r_{f}^{-1 / 3}\left(I_{3 \times 3}+\left(r_{f}-1\right) N \otimes N\right) .
\end{aligned}
$$

The parameters $r_{0}, r_{f} \geq 1$ quantify the degree of anisotropy at the initial and final temperature respectively. They describe the extent to which the material tends to deform in the directions $N_{0}$ and $N$ respectively. We envision that $r_{0}, r_{f}$ arise from evaluating some underlying, monotone decreasing function $\bar{r}(T)$ at the temperatures $T=T_{0}$ and $T=T_{f}$.

Remark 1.1. (i) The constant -3 in (1.2) is chosen so that $\min W_{e}=0$.

(ii) The elastic energy $\mathcal{I}_{N_{0}^{h}}^{h}$ is defined without any displacement or traction boundary conditions as we are dealing actuation only.

(iii) The temperature-dependent function $\bar{r}(T)$ satisfies $\lim _{T \rightarrow \infty} \bar{r}(T)=1$ because nematic materials behave isotropically at large temperature. Indeed, setting $r_{0}=r_{f}=1$ in the formulae above, one recovers the standard incompressible neo-Hookean energy for isotropic materials.

(iv) In the definition (1.1) of $\mathcal{I}_{N_{0}^{h}}^{h}\left(Y^{h}\right)$ we imposed the kinematic constraint $N^{h}=\frac{\left(\nabla Y^{h}\right) N_{0}^{h}}{\left|\left(\nabla Y^{h}\right) N_{0}^{h}\right|}$. There are nematic elastomers which do not satisfy this kinematic constraint (i.e. where the director $N$ is allowed to vary more freely). Those materials can show macroscopic deformations which 
arise from the fine-scale microstructure produced by oscillations of $N$ [13],[14],[15],[21] (see also the experiments by Kundler and Finkelmann [28]).

In the present paper, we are interested in actuating complex, yet predictable, shape by programming an initial heterogeneous anisotropy $N_{0}^{h}$ in the nematic elastomer. It would be difficult to control actuation for a material that is capable of freely forming microstructure, which competes with the shape change driven by the programmed anisotropy, even at low energy. Thus, in order to produce "controlled heterogeneity" in actuation, we impose the kinematic constraint $N^{h}=\frac{\left(\nabla Y^{h}\right) N_{0}^{h}}{\left|\left(\nabla Y^{h}\right) N_{0}^{h}\right|}$. The constraint is similar to one that was imposed by Modes et al. [31] in their prediction for conical and saddle like actuation in nematic glass sheets with radial and azimuthal heterogeneity (in fact, both constraints are equivalent for zero energy/stress free states; see Proposition A.4).

(v) We have neglected Frank elasticity and related effects in our model, as these are expected to be small in comparison to the entropic elasticity (see discussion in Chapter 3 in Warner and Tarentjev [43]). However, to derive the key metric constraint (introduced below) as a necessary feature of low energy deformations, we add to the energy (1.1) a small contribution from Frank elasticity for technical reasons. This is discussed in Section 1.6.

Notation. So far, all the vector fields we considered were maps $\Omega_{h} \rightarrow \mathbb{R}^{3}$ and we denoted them by capital letters, e.g. $N_{0}^{h}, Y^{h}$. Throughout the paper, we will also consider vector fields defined on the midplane $\omega \subset \mathbb{R}^{2}$ and we denote these by lowercase letters, e.g. $n_{0}, y: \omega \rightarrow \mathbb{R}^{2}$. Moreover, we use $(\tilde{\cdot})$ to distinguish the two-dimensional midplane variables from the three-dimensional ordinary variables, that is

$$
x=\left(x_{1}, x_{2}, x_{3}\right), \quad \tilde{x}=\left(x_{1}, x_{2}\right) \quad \tilde{\nabla}=\left(\partial_{x_{1}}, \partial_{x_{2}}\right) .
$$

We will only consider $x \in \Omega_{h}$ and so $x_{3} \in(-h / 2, h / 2)$ is small.

\subsection{The metric constraint and overview of results}

We have the following situation in mind: An initial director field $N_{0}^{h}$ is heterogeneously programmed on the nematic sheet $\Omega_{h}$ at the initial temperature $T_{0}$. As $T$ is varied from $T_{0}$ to the final temperature $T_{f}$, the sheet undergoes a non-uniform spontaneous deformation. We postulate that the spontaneous deformations are those that minimize the elastic energy $\mathcal{I}_{N_{0}^{h}}^{h}$.

Our goal is to characterize the class of director fields $N_{0}^{h}$ and corresponding deformations $Y^{h}$ which yield small elastic energy $\mathcal{I}_{N_{0}^{h}}^{h}\left(Y^{h}\right)$. This characterization comes in the form of a twodimensional effective metric constraint (1.6). To see how this arises, we first consider a naive approach by requiring $\mathcal{I}_{N_{0}^{h}}^{h}\left(Y^{h}\right)=0$ (recall that min $W^{e}=0$ ). By Proposition A.4, $\mathcal{I}_{N_{0}^{h}}^{h}\left(Y^{h}\right)=0$ is equivalent to

$$
\left(\nabla Y^{h}\right)^{T} \nabla Y^{h}=r^{-1 / 3}\left(I_{3 \times 3}+(r-1) N_{0}^{h} \otimes N_{0}^{h}\right)=: \ell_{N_{0}^{h}} \quad \text { a.e. on } \Omega_{h},
$$

where $r=r_{f} / r_{0}$ so that $r \in(0,1)$ for heating and $r>1$ for cooling. However, (1.5) is too strong of a condition to be useful, meaning that there are only few choices of $N_{0}^{h}$ for which a $Y^{h}$ satisfying (1.5) exists. We explain this in Remark 1.2 below.

Given that (1.5) is too restrictive, we relax the problem and study approximate minimizers of the elastic energy $\mathcal{I}_{N_{0}^{h}}^{h}\left(Y^{h}\right)$. The key observation is that by making use of the thinness of the sheet $\Omega_{h}$ and assuming that $N_{0}^{h}$ does not vary too much as a function of $x_{3}$, one can show that 


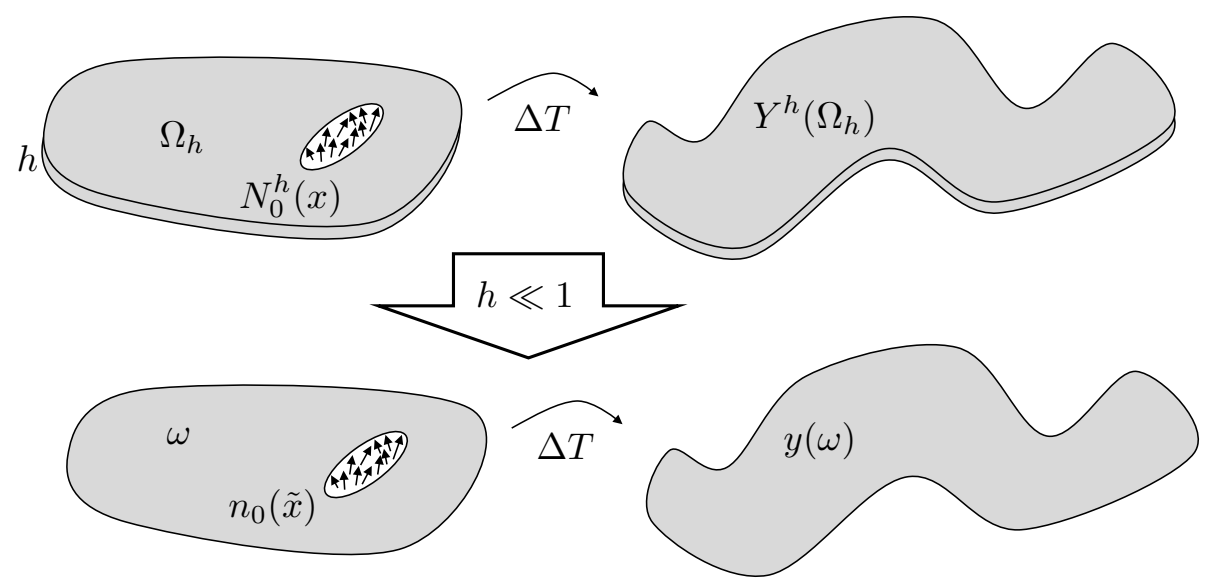

Figure 1: Actuation for thin sheets is characterized by the midplane fields.

approximate minimizers are characterized (in a sense to be made precise) by the following effective metric constraint (1.6). It is a two-dimensional reduction of the three-dimensional constraint (1.5) and reads

$$
(\tilde{\nabla} y)^{T} \tilde{\nabla} y=r^{-1 / 3}\left(I_{2 \times 2}+(r-1) \tilde{n}_{0} \otimes \tilde{n}_{0}\right)=: \tilde{\ell}_{n_{0}} \quad \text { a.e. on } \omega
$$

Here we used the notation introduced above, so $y, n_{0}: \omega \rightarrow \mathbb{R}^{3}$ are essentially the restrictions of $Y^{h}, N_{0}^{h}$ to the midplane $\omega$ (i.e. $\left.y\left(x_{1}, x_{2}\right) \approx Y^{h}\left(x_{1}, x_{2}, 0\right)\right)$ and $(\tilde{)})$ refers to the projection onto midplane variables, i.e. $\tilde{\nabla} y$ is a $3 \times 2$ matrix and $\tilde{n}_{0} \in B_{1}(0) \subset \mathbb{R}^{2}$ is the projection of $n_{0}$ onto $\omega$.

The metric constraint (1.6) appeared in our earlier short paper [39] with a view towards applications. In the present paper, we discuss the metric constraint from a mathematical perspective.

Remark 1.2. Let us explain why (1.5) is too restrictive. Assuming that $N_{0}^{h}$ is sufficiently smooth (the non-smooth case is treated in Lewicka and Pakzad [30]), there exists a $Y^{h}$ satisfying (1.5) if and only if the components of the Riemann curvature tensor of $\ell_{N_{0}^{h}}$ vanish. This condition is well-known in the physics literature (e.g. Efrati et al. [22]), and in the language of continuum mechanics; it gives compatibility of the Right Cauchy-Green deformation tensor (e.g. Blume [12]). As a consequence, $N_{0}^{h}$ has to satisfy a certain nonlinear partial differential equation, and so it must come from a very restricted set of functions.

We will now discuss the precise context in which (1.6) arises; that is under which circumstances the midplane vector fields characterize approximate minimizers of the strain energy (and thus give a recipe for design). The intuition is expressed by Figure 1.

It is clear that we have to assume that the full director field $N_{0}^{h}$ does not vary too much away from the midplane. For the following, recall our notation $n_{0}\left(x_{1}, x_{2}\right) \approx N_{0}^{h}\left(x_{1}, x_{2}, 0\right)$ and $\tilde{x}=\left(x_{1}, x_{2}\right)$.

Assumption 1.3. We assume

$$
N_{0}^{h}(x)=n_{0}(\tilde{x})+O(h), \quad \text { for a.e. } x \in \Omega_{h}, \quad \text { i.e., }\left\|N_{0}^{h}-n_{0}\right\|_{L^{\infty}\left(\Omega_{h}\right)} \leq \tau h \text { for some } \tau>0 .
$$

The $O(h)$ term accounts for the following two possible deviations from the midplane field. For definiteness, we have fixed the maximum tolerance $\tau>0$ for these non-idealities.

(a) It accounts for deviations of the director field through the thickness, which are of the same order as the thickness. Note that this excludes twisted or splay-bend nematic sheets [25],[42], 
for which one prescribes the director field on the top surface of the sheet and then differently then on the bottom surface, so that the director field has to vary by an $O(1)$ amount through the thickness.

(b) It accounts for the possibility of planar deviations. In the synthesis techniques employed by Ware et. al. [41], the director field is prescribed in voxels or cubes whose characteristic length is similar to the thickness and we expect the experimental error to be of this order.

From now on we make Assumption 1.3. We would like to use the metric constraint (1.6) to characterize approximate minimizers of the strain energy as $h \rightarrow 0$. Such a characterization would ideally contain two parts; namely (1.6) should be a sufficient condition and a necessary condition for being an approximate minimizer. (Ideally, this corresponds to the two statements which would comprise $\Gamma$-convergence to a two-dimensional energy functional, though we do not quite prove such a convergence.) The main results in our paper make progress in both of these directions. To put the results in context, we note that generic deformations have energy $\mathcal{I}_{N_{0}^{h}}^{h}\left(Y^{h}\right)=O(h)$, so strain energies of order $\ll h$ can be reasonably considered small.

The main results are as follows.

(i) Sufficiency of the metric constraint. Given midplane fields $\left(y, n_{0}\right)$ satisfying the metric constraint (1.6) and for sheets of sufficiently small thickness $h$, we can construct global deformations $Y^{h}: \Omega_{h} \rightarrow \mathbb{R}^{3}$ which approximately minimize the strain energy and satisfy $Y^{h}(\tilde{x}, 0) \approx y(\tilde{x})$ in the appropriate Sobolev norm. It turns out that the correct energy scale of an "approximate minimizer" is related to the smoothness of the design. That is, for nonisometric origami (piecewise constant design) as in Figure 13, our constructions satisfy

$$
\mathcal{I}_{N_{0}^{h}}^{h}\left(Y^{h}\right) \leq O\left(h^{2}\right) \quad(\text { Theorem 1.4), }
$$

and for sufficiently smooth surfaces such as the lifted surfaces in Figure 2, our constructions satisfy

$$
\mathcal{I}_{N_{0}^{h}}^{h}\left(Y^{h}\right) \leq O\left(h^{3}\right) \quad(\text { Theorem } 1.8 \text { and Corollary 1.9) }
$$

The techniques employed here are akin to those of Conti and Dolzmann [16],[17] for incompressibility and Conti and Maggi [18] for nonisometric origami.

(ii) Optimality of nonisometric origami. We also have a result concerning the optimality of (1.8) for nonisometric origami: We consider a two dimensional analog to the entropic elasticity $\left(\tilde{\mathcal{I}}_{n_{0}}^{h}\right.$ in equation (1.18) below), and show in this setting that interfaces separating regions of distinct constant director necessarily incur an elastic penalty of $O\left(h^{2}\right)$ upon actuation (Theorem 1.6).

(iii) Necessity of the metric constraint. We prove that the metric (1.6) is necessary for pure bending if we augment the entropic elasticity $\mathcal{I}_{N_{0}^{h}}^{h}$ in (1.1) with an approximation of Frank elasticity which is natural to nematics (Theorem 1.12). Specifically, if $\mathcal{I}_{N_{0}^{h, \varepsilon}}^{h,}$ is the sum of a nondimensionalized version of $\mathcal{I}_{N_{0}^{h}}^{h}$ and an additional term approximating Frank elasticity with lengthscale $\varepsilon$ (equation (1.28) below) which is comparable to thickness (i.e. $\varepsilon \equiv O(h)$ ), then using the results of Friesecke, James and Müller [23] on Geometric Rigidity, we show that bending configurations satisfying $\mathcal{I}_{N_{0}^{h}}^{h, \varepsilon_{h}}\left(Y^{h}\right) \leq O\left(h^{3}\right)$ must be characterized by sufficiently smooth midplane fields satisfying (1.6). 
We note that there are, in general, multiple deformations $y$ which satisfy (1.6) for a given $n_{0}$ (e.g. the sheet can actuate upward or downwards in different places). We imagine that one can distinguish between these by appropriately breaking additional symmetries, but we do not investigate this further.

The constraint (1.6) generalizes a metric constraint that has been proposed by Aharoni et al. [1] for actuation of nematic sheets. Indeed, (1.6) is more general in that (a) it need only hold almost everywhere, allowing for piecewise constant director designs and (b) the director can be programmed out of plane. At the same time, it is easy to see that (1.6) reduces to the contraint [1] for smooth planar director fields. (With $n_{0} \equiv \tilde{n}_{0}$, we can write $\tilde{n}_{0} \cdot e_{1}=\cos (\theta)$ and $\tilde{n}_{0} \cdot e_{2}=\sin (\theta)$ for a Cartesian basis $\left\{e_{1}, e_{2}\right\} \subset \mathbb{R}^{2}$ on the plane. It follows that $(\tilde{\nabla} y)^{T} \tilde{\nabla} y=\tilde{\ell}_{n_{0}}=\tilde{R}(\theta) \operatorname{diag}\left(r^{2 / 3}, r^{-1 / 3}\right) \tilde{R}(\theta)^{T}$ for $\tilde{R}(\theta) \in S O(2)$ a rotation of $\theta$ about the normal to the initially flat sheet as required by [1].)

\subsection{Nonisometric origami constructions under the metric constraint}

We consider continuous, piecewise affine deformations (nonisometric origami) and we prove that if these satisfy the effective metric constraint (1.6), then their strain energy scales at most like $h^{2}$.

By definition a nonisometric origami is a union of a finite number of polygonal regions $\omega_{\alpha}$ which each have a constant director field, i.e.,

$$
\begin{aligned}
& \omega=\bigcup_{\alpha=\{1, \ldots, N\}} \omega_{\alpha}, \quad \omega_{\alpha} \text { mutually disjoint and polygonal, } \\
& n_{0}: \omega \rightarrow \mathbb{S}^{2} \quad \text { satisfies } \quad n_{0}(\tilde{x}) \equiv n_{0 \alpha}, \quad\left(\tilde{x} \in \omega_{\alpha}, \forall \alpha \in\{1, \ldots, N\}\right), \\
& \tilde{n}_{0 \alpha} \neq \pm \tilde{n}_{0 \beta} \quad \text { when a } g_{\alpha \beta} \in \mathbb{S}^{1} \text { interface connects } \omega_{\alpha} \text { and } \omega_{\beta}, \alpha \neq \beta .
\end{aligned}
$$

The last condition is only there to ensure that each interface corresponds to a non-trivial change of the director (otherwise that interface would be superfluous). Examples of nonisometric origami are presented in Figure 13 in the appendix.

Our main result for nonisometric origami says that, given a (continuous, piecewise affine) midplane deformation $y: \omega \rightarrow \mathbb{R}^{3}$ satisfying the effective metric constraint (1.6), we can construct map

$Y^{h}: \Omega_{h} \rightarrow \mathbb{R}^{3}$ which has the strain energy $\mathcal{I}_{N_{0}^{h}}^{h}\left(Y^{h}\right) \leq O\left(h^{2}\right)$. (We think of $Y^{h}$ as an approximate minimizer of the strain energy, especially given the optimality result of the $O\left(h^{2}\right)$ scaling proved later.) Moreover, the restriction of the map $Y^{h}$ to the midplane is close to the original map $y$ (in the appropriate Sobolev norm) and in this sense $Y^{h}$ is an approximate extension of $y$. We comment further on the additional technical assumption that $y$ has a $\delta$-smoothing after the theorem; for now we just note that it is satisfied in several exemplary cases.

Theorem 1.4. Let $\omega$ and $n_{0}$ be a nonisometric origami as defined above, and let $N_{0}^{h}: \Omega_{h} \rightarrow \mathbb{S}^{2}$ be any vector field that is close to $n_{0}$ in the sense of (1.7). Let $y \in W^{1, \infty}\left(\omega, \mathbb{R}^{3}\right)$ be a piecewise affine midplane deformation such that

$$
\begin{aligned}
y(\tilde{x}) & =\tilde{F}_{\alpha} \tilde{x}+c_{\alpha}, \\
\left(\tilde{F}_{\alpha}\right)^{T} \tilde{F}_{\alpha} & =\tilde{\ell}_{n_{0 \alpha}},
\end{aligned}
$$

for all $\tilde{x} \in \omega_{\alpha}$ and all $\alpha \in\{1, \ldots, N\}$. Suppose further that for all small enough $\delta>0, y$ has a $\delta$-smoothing $y^{\delta}$ in the sense of Definition 1.5 below.

Then, there exists an $m>0$ such that if we set $\delta_{h}=m h$, then for all small enough $h>0$ there exists a map $Y^{h}: \Omega_{h} \rightarrow \mathbb{R}^{3}$ with

$$
Y^{h}(\tilde{x}, 0)=y^{\delta_{h}}(\tilde{x}), \quad(\tilde{x} \in \omega),
$$




$$
\mathcal{I}_{N_{0}^{h}}^{h}\left(Y^{h}\right) \leq O\left(h^{2}\right)
$$

Moreover, $Y^{h}$ is an approximate extension of $y$ in the sense that $\left\|y^{\delta_{h}}-y\right\|_{W^{1,2}\left(\omega, \mathbb{R}^{3}\right)} \leq O(h)$.

Theorem 1.4 is proved in Section 3.3. In the statement we assumed that $y$ has a $\delta$-smoothing in the following sense:

Definition 1.5. We say that $y: \omega \rightarrow \mathbb{R}^{3}$ has a $\delta$-smoothing, if there exists a map $y^{\delta} \in C^{3}\left(\bar{\omega}, \mathbb{R}^{3}\right)$ and a subset $\omega_{\delta} \subset \omega$ of area less than $C \delta$ such that

$$
\begin{array}{r}
y^{\delta}=y \text { on } \omega \backslash \omega_{\delta}, \quad\left\|\partial_{1} y^{\delta} \times \partial_{2} y^{\delta}\right\|_{L^{\infty}} \geq c>0, \\
\left\|\tilde{\nabla} y^{\delta}\right\|_{L^{\infty}} \leq C, \quad\left\|\tilde{\nabla} \tilde{\nabla} y^{\delta}\right\|_{L^{\infty}} \leq C \delta^{-1}, \quad\left\|\tilde{\nabla}^{(3)} y^{\delta}\right\|_{L^{\infty}} \leq C \delta^{-2}
\end{array}
$$

for some constants $C, c>0$ which can depend on $y$ and $\omega$ but not on $\delta$.

The existence of such a $\delta$-smoothing (of the only Lipschitz continuous midplane deformation $y$ ) is an important technical tool. It is needed because the global deformation $Y^{h}$ has to satisfy the incompressibility constraint $\operatorname{det} \nabla Y^{h}=1$. (Essentially, the non-degeneracy of the derivatives of $y^{\delta}$ allows one to employ the inverse function theorem to derive a sufficiently well-behaved ordinary differential equation as described in section 3.)

This technical issue has appeared in previous works on incompressibility (also, a $\operatorname{det} F>0$ constraint) in thin sheets. It was first appreciated by Belgacem [5] and later addressed in some generality by Trabelsi [40] and Conti and Dolzmann [16]. However, their methods are very geometrical in nature (they are largely based on Whitney's ideas on the singularities of functions $\mathbb{R}^{n} \rightarrow \mathbb{R}^{2 n-1}$ ) and it is not obvious how to extract from them the $\delta$-dependent control of the higher derivatives which we need in the present context.

We discuss several examples of nonisometric origami in an extensive appendix $\mathbf{C}$. The examples are depicted in Figure 13 and include a construction which will fold into a box, originally due to [33], as well as further examples which previously appeared in a companion paper to this one [39] that was geared towards a physics audience. We also discuss in some detail an equivalent formulation of the metric constraint (1.13) for nonisometric origami in terms of compatibility conditions. These are akin to the rank-one condition studied in the context of fine-scale twinning during the austenite martensite phase transition and actuation active martensitic sheets [2],[8],[9] and to the recently studied compatibility conditions for the actuation for nematic elastomer and glass sheets using planar programming of the director [32],[33].

Importantly, we prove that all of the examples of nonisometric origami considered in this work indeed have a $\delta$-smoothing, in the sense of Definition 1.5, by using explicit constructions (see Sections 2.1-2.4). We leave it as an interesting open problem to prove that any nonisometric origami possesses a $\delta$-smoothing.

\subsection{On the optimality of nonisometric origami}

From Theorem 1.4, we can construct approximations to nonisometric origami (under the hypothesis (1.16)) with energy $O\left(h^{2}\right)$. Thus, it is natural to ask whether these constructions are energetically optimal for prescribed director field. Following the work of Kohn and Müller [27] and others [7], [18], optimality in our context would be established by

$$
\inf \left\{\mathcal{I}_{N_{0}^{h}}^{h}\left(Y^{h}\right): Y^{h} \in W^{1,2}\left(\Omega_{h}, \mathbb{R}^{3}\right)\right\} \geq c_{L} h^{2}
$$


for all small enough $h>0$. Here $\omega$ and $n_{0}$ describe a nonisometric origami (defined in the last section) and $N_{0}^{h}$ is close to $n_{0}$ in the sense of (1.7).

In the present paper, we prove the analogous lower bound for a two-dimensional analogue of the three-dimensional entropic strain energy,

$$
\tilde{\mathcal{I}}_{n_{0}}^{h}(y)=h \int_{\omega}\left(\left|(\tilde{\nabla} y)^{T} \tilde{\nabla} y-\tilde{\ell}_{n_{0}}\right|^{2}+h^{2}|\tilde{\nabla} \tilde{\nabla} y|^{2}\right) d \tilde{x}
$$

The first term here represents membrane stretching part and is minimized exactly when the metric constraint (1.6) is satisified. The second term approximates bending. Such a two-dimensional energy is a widely used proxy to describe the elasticity of non-Euclidean plates (e.g. Efrati et al. [22] and Bella and Kohn [6]). In a broader context, these proxies often agree in $h$-dependent optimal energy scaling with that of the three dimensional elastic energy, and deformations which achieve this scaling in this two dimensional setting tend to form the midplane deformations for optimal three dimensional constructions (e.g. Bella and Kohn [7] and the single fold approximation of Conti and Maggi [18]).

For the effective two-dimensional theory, we prove a lower bound with the expected $O\left(h^{2}\right)$ scaling.

Theorem 1.6. Let $r>0$ and $\neq 1, \omega$ satisfy (1.9), and $n_{0}$ as in (1.10) and (1.11). For $h>0$ sufficiently small

$$
\inf \left\{\tilde{\mathcal{I}}_{n_{0}}^{h}(y): y \in W^{2,2}\left(\omega, \mathbb{R}^{3}\right)\right\} \geq c_{L} h^{2}
$$

Here, $c_{L}=c_{L}\left(n_{0}, r, \omega\right)>0$ is independent of $h$.

Theorem 1.6 is proved in Section 4 .

Remark 1.7. (i) If, in addition to the assumptions of the theorem, there exists a $y \in W^{1, \infty}\left(\omega, \mathbb{R}^{3}\right)$ satisfying (1.12) and each $\tilde{F}_{\alpha}$ and $n_{0 \alpha}$ in these relations satisfies (1.13), then for $h>0$ sufficiently small, there exists a $y^{h} \in C^{3}\left(\bar{\omega}, \mathbb{R}^{3}\right)$ such that

$$
\left\|y^{h}-y\right\|_{W^{1,2}} \leq O(h) \quad \text { and } \quad \tilde{\mathcal{I}}_{n_{0}}^{h}\left(y^{h}\right) \leq O\left(h^{2}\right) .
$$

Hence, nonisometric origami is optimal in this two dimensional setting. The proof of this is straightforward. Indeed, the estimates (1.16), with exception to the full-rank condition, can be obtained by standard mollification (for more details, see Section 2). Setting $\delta=h$ for these estimates yields a $y^{h}$ satisfying (1.19).

(ii) Let us discuss some of the heuristics behind the lower bound in Theorem 1.6 and the conjectured lower bound (1.17). At an interface separating two regions of distinct constant director, an energetic penalty associated with membrane stretching at $O(h)$ drives the deformation to be piecewise affine with a fold precisely at the interface connecting the two regions, whereas an energetic penalty associated with bending at $O\left(h^{3}\right)$ cannot accommodate sharp folds, and thus a smoothing is necessitated. This interplay gives rise to an intermediate energetic scaling between $O(h)$ and $O\left(h^{3}\right)$. For isometric origami, folds can be smoothed to mostly preserve the isometric condition, leading to approximate constructions and (under suitable hypothesis) lowerbounds which scale as $O\left(h^{8 / 3}\right)$ (see, for instance, Conti and Maggi [18]). For nonisometric origami, the preferred metric jumps across a possible fold and this leads to a larger membrance stretching term. 


\subsection{Examples of pure bending actuation under the metric constraint}

We turn now to the case of smooth or sufficiently smooth surfaces and programs satisfying the metric constraint (1.6). For these configurations, we will show the actuation is pure bending, i.e. $O\left(h^{3}\right)$ in the entropic strain energy after actuation.

Below, we give a general result that gives $O\left(h^{3}\right)$ scaling for sufficiently smooth $y, n_{0}$. Before we state that result (Corollary 1.9), we present a large class of $y, n$ which automatically satisfy the two-dimensional metric constraint (1.6). These surfaces are given as the graph of a function, combined with an appropriate contraction (here we consider cooling, so $r>1$ ). We call these "lifted surfaces". They are defined by

$$
y(\tilde{x})=r^{-1 / 6}\left(x_{1} e_{1}+x_{2} e_{2}\right)+\varphi\left(r^{-1 / 6} \tilde{x}\right) e_{3} .
$$

where the function $\varphi$ is from the following set

$$
\left\{\phi \in W^{2, \infty}\left(r^{-1 / 6} \omega, \mathbb{R}\right):\|\tilde{\nabla} \phi\|_{L^{\infty}}<\lambda_{r}:=r-1, \quad \operatorname{supp} \phi \subset r^{-1 / 6} \omega_{m}\right\} .
$$

Here, we set $\omega_{m}:=\{\tilde{x} \in \omega: \operatorname{dist}(\tilde{x}, \partial \omega)>m>0\}$ (recall that $\omega \subset \mathbb{R}^{2}$ is the midplane of the sheet, a bounded Lipschitz domain). The corresponding director field of a lifted surface is

$$
n_{0}(\tilde{x})=\frac{1}{\lambda_{r}^{1 / 2}}\left(\begin{array}{c}
\partial_{1} \varphi\left(r^{-1 / 6} \tilde{x}\right) \\
\partial_{2} \varphi\left(r^{-1 / 6} \tilde{x}\right) \\
\left(\lambda_{r}-\left|\tilde{\nabla} \varphi\left(r^{-1 / 6} \tilde{x}\right)\right|^{2}\right)^{1 / 2}
\end{array}\right) .
$$

We emphasize again that any such choice of $y, n_{0}$ satisfies (1.6). This fact can be proved by rewriting (1.6) in an equivalent form, which is in fact more practical from the perspective of design and we discuss this below. The first main result is then that lifted surfaces have entropic energy of $O\left(h^{3}\right)$ (and therefore they are good candidates for designable actuation).

Theorem 1.8 (Lifted Surfaces). Let $r>1$ and $m>0$. Given a midplane deformation $y$ as in (1.20) with $\varphi$ taken from the set (1.21), define the director field $n_{0}$ as in (1.22). Let $N_{0}^{h}$ be close to $n_{0}$ in the sense of (1.7).

Then, for every $h>0$ sufficiently small, there exists a $y^{h} \in C^{3}\left(\bar{\omega}, \mathbb{R}^{3}\right)$ and an extension $Y^{h} \in$ $C^{1}\left(\bar{\omega}, \mathbb{R}^{3}\right)$ such that

$$
Y^{h}(\tilde{x}, 0)=y^{h}(\tilde{x}), \quad \tilde{x} \in \omega, \quad\left\|y^{h}-y\right\|_{W^{1, \infty}(\omega)} \leq O(h), \quad \mathcal{I}_{N_{0}^{h}}^{h}\left(Y^{h}\right) \leq O\left(h^{3}\right) .
$$

The key reason why lifted surfaces satisfy the $O\left(h^{3}\right)$ scaling is that they satisfy the metric constraint and are sufficiently smooth. Thus, we can generalize the proof of Theorem 1.8 to obtain the following result. (Here $y$ is assumed to be smoother than it has to be for lifted surfaces smooth and so one does not need to introduce the smoothing $y^{h}$ as in the theorem above.)

Corollary 1.9 (Smooth Surfaces). Let $r \in(0,1)$ or $r>1$. Let $n_{0}$ satisfy (1.7). If $y \in C^{3}\left(\bar{\omega}, \mathbb{R}^{3}\right)$ and $n_{0} \in C^{2}\left(\bar{\omega}, \mathbb{S}^{2}\right)$ such that $(\tilde{\nabla} y)^{T} \tilde{\nabla} y=\tilde{\ell}_{n_{0}}$ everywhere on $\omega$, then for $h>0$ sufficiently small, there exists a $Y^{h} \in C^{1}\left(\bar{\Omega}_{h}, \mathbb{R}^{3}\right)$ such that

$$
Y^{h}(\tilde{x}, 0)=y(\tilde{x}), \quad \tilde{x} \in \omega \quad \mathcal{I}_{N_{0}^{h}}^{h}\left(Y^{h}\right) \leq O\left(h^{3}\right) .
$$

The results stated here are proved in section 3.4.

The idea of lifted surfaces is based on an equivalent rewriting of the metric constraint $(\tilde{\nabla} y)^{T} \tilde{\nabla} y=$ $\tilde{\ell}_{n_{0}}$. (This equivalent form also yields a concrete design scheme for the actuation of nematic elastomers sheets.) Essentially, we take the picture of $y$ being a solution to $(\tilde{\nabla} y)^{T} \tilde{\nabla} y=\tilde{\ell}_{n_{0}}$ defined by a 
predetermined $n_{0}$ and turn it on its head. That is, we first identify the set of deformation gradients that are consistent with (1.6) for any director field and then we identify the director associated with that deformation gradient.

Proposition 1.10. Let $r>1$. The metric constraint (1.6) holds if and only if

$$
\tilde{\nabla} y(\tilde{x})=\left(\partial_{1} y \mid \partial_{2} y\right)(\tilde{x}) \in \mathcal{D}_{r}, \quad n_{0}(\tilde{x}) \in \mathcal{N}_{\tilde{\nabla} y(\tilde{x})}^{r} \quad \text { a.e. } \tilde{x} \in \omega .
$$

Here,

$$
\begin{gathered}
\mathcal{D}_{r>1}=\left\{\tilde{F} \in \mathbb{R}^{3 \times 2}:|\tilde{F}|^{2} \leq r^{-1 / 3}+r^{2 / 3}, r^{-1 / 3} \leq\left|\tilde{F} \tilde{e}_{\alpha}\right|^{2} \leq r^{2 / 3}, \alpha=1,2,\right. \\
\left.\left(\tilde{F} \tilde{e}_{1} \cdot \tilde{F} \tilde{e}_{2}\right)^{2}=\left(\left|\tilde{F} \tilde{e}_{1}\right|^{2}-r^{-1 / 3}\right)\left(\left|\tilde{F} \tilde{e}_{2}\right|^{2}-r^{-1 / 3}\right)\right\}
\end{gathered}
$$

and

$$
\begin{aligned}
\mathcal{N}_{\tilde{F}}^{r>1}=\left\{m \in \mathbb{S}^{2}:\right. & \left(m \cdot e_{\alpha}\right)^{2}=\frac{\left|\tilde{F} \tilde{e}_{\alpha}\right|^{2}-r^{-1 / 3}}{r^{2 / 3}-r^{-1 / 3}} \alpha=1,2, \\
& \left.\operatorname{sign}\left(\left(m \cdot e_{1}\right)\left(m \cdot e_{2}\right)\right)=\operatorname{sign}\left(\tilde{F} \tilde{e}_{1} \cdot \tilde{F} \tilde{e}_{2}\right)\right\}
\end{aligned}
$$

for sign: $\mathbb{R} \rightarrow\{-1,0,1\}$ the sign function with $\operatorname{sign}(0)=0$. (For $r<1$, the inequalities in (1.24) and the sign in (1.25) are reversed.)

We prove this equivalence in the appendix, see Proposition B.1. There, we also show that for any $\tilde{F} \in \mathcal{D}_{\bar{r}}$, there exists an $m \in \mathcal{N}_{\tilde{F}}^{r}$. This means that for characterizing the geometry of surfaces which satisfy the metric constraint (1.6), we need only to consider the set of deformation gradients from a flat sheet $\omega$ which satisfy $\tilde{\nabla} y(\tilde{x}) \in \mathcal{D}_{r}$ a.e. $\tilde{x} \in \omega$. Unfortunately, such a broad characterization remains open. Of particular difficulty is the fact that this condition on the deformation gradient implies the equality

$$
\left(\partial_{1} y \cdot \partial_{2} y\right)^{2}=\left(\left|\partial_{1} y\right|^{2}-r^{-1 / 3}\right)\left(\left|\partial_{2} y\right|^{2}-r^{-1 / 3}\right), \quad \text { a.e. on } \omega .
$$

Lifted surfaces constitute a broad class of deformations such that this constraint holds trivially.

Remark 1.11. (i) The surfaces of revolution in Aharoni et al. [1] and the designs exploring Gaussian curvature in Mostajeran [36] satisfy the conditions of Corollary 1.9. Thus, these designs and their predicted actuation are pure bending configurations in that they have entropic energy of $O\left(h^{3}\right)$ (which justifies that they are good candidates to be realized in actuation).

(ii) The lifted surfaces ansatz allows for actuation of a large variety of shapes, since the limitations imposed by (1.21) are not very restrictive. Since $r$ can be significantly different from 1 in nematic elastomers, one can form shapes with significant displacement like spherical caps and sinusoidally rough surfaces. Figure 2 shows two additional examples with complex surface relief. These are but a small sample of the designs amenable to this framework. Indeed, given any arbitrary greyscale image $\mathcal{G}$, we can program a nematic sheet so that the surface of the sheet upon cooling corresponds to this image. We do this by smearing $\mathcal{G}$ (for instance by mollification or by averaging over a small square twice) and taking this as $\varphi$.

(iii) The key ingredient to the design of lifted surfaces is the ability to program the director three dimensionally. However, to our knowledge, experimental studies on nematic elastomer sheets such as Ware et. al. [41] have examined planar inscription of the director only. While we 


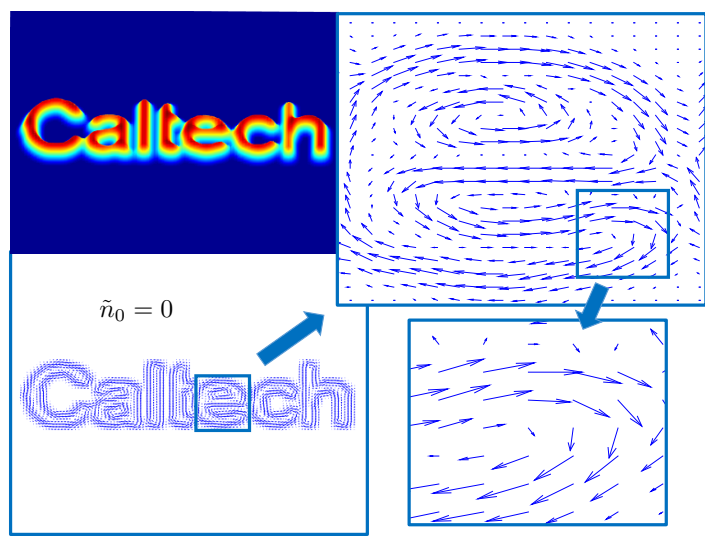

(a) Caltech

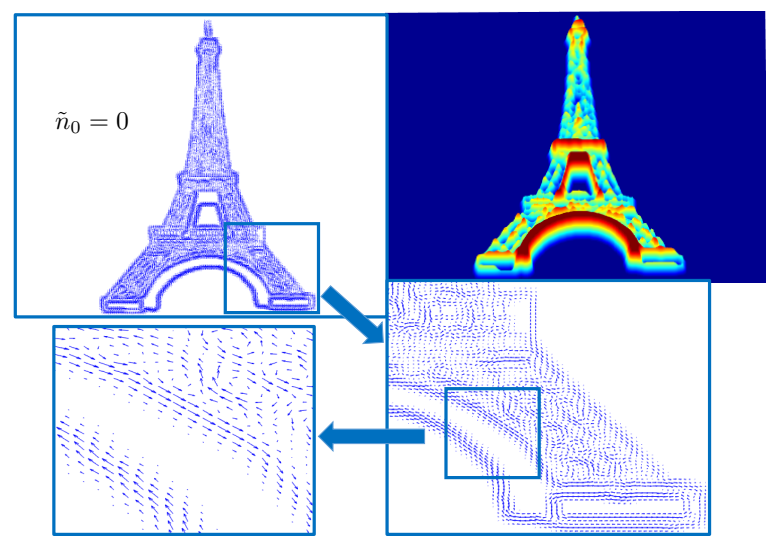

(b) Eiffel Tower

Figure 2: The deformed shape and designs for lifted surfaces. The planar part of the director $\tilde{n}_{0}$ is plotted.

hope that promising designs such as lifted surfaces will inspire future experimentation to realize three dimensional programming, in any case, the theory and design scheme are easily adapted to the planar case. Specifically, the metric constraint (1.6) reduces to the metric underlying Aharoni et al. [1] in the case of a planar program.

(iv) To arrive at the results presented in this section (fully detailed in Section 3.4), we employ techniques of Conti and Dolzmann [16], [17] to construct incompressible three dimensional deformations $Y^{h} \in C^{1}\left(\bar{\Omega}_{h}, \mathbb{R}^{3}\right)$. These techniques rely on the ability to approximate Sobolev functions by sufficiently smooth functions (see Section 3.1). In this direction, an important feature of lifted surfaces is that given any $y$ as in (1.20) with $\varphi$ as in (1.21), there exists a smooth $y^{h}$ approximating $y$ in the $W^{2,2}\left(\omega, \mathbb{R}^{3}\right)$ norm which additionally satisfies $\tilde{\nabla} y^{h} \in \mathcal{D}_{r}$ on $\omega$. The space $\mathcal{D}_{r}$ can be thought of as the appropriate generalization to nematic anisotropy of the space of matrices representing isometries. Specifically, in the isotropic case $r=1, \mathcal{D}_{r}$ reduces to $\mathcal{D}_{1}=\left\{\tilde{F} \in \mathbb{R}^{3 \times 2}: \tilde{F}^{T} \tilde{F}=I_{2 \times 2}\right\}$. The corresponding function space

$$
W_{i s o}^{2,2}\left(\omega, \mathbb{R}^{3}\right):=\left\{y \in W_{i s o}^{2,2}\left(\omega, \mathbb{R}^{3}\right):(\tilde{\nabla} y)^{T} \tilde{\nabla} y=I_{2 \times 2} \quad \text { a.e. }\right\}
$$

has been extensively studied in the literature as this is the space of all bending deformations for isotropic sheets (as detailed rigorously by Friesecke et. al. [23]). For instance, Pakzad [37] showed that smooth isometric immersions are dense in $W_{i s o}^{2,2}$ as long as the initially flat sheet $\omega$ is a convex regular domain. This was later generalized by Hornung [26] for flat sheets which belong to a much larger class of bounded and Lipschitz domains. For nematic elastomers, an appealing analogue to these results would be a similar density result for the space

$$
W_{r}^{2,2}\left(\omega, \mathbb{R}^{3}\right):=\left\{y \in W^{2,2}\left(\omega, \mathbb{R}^{3}\right): \tilde{\nabla} y \in \mathcal{D}_{r} \quad \text { a.e. }\right\} .
$$

For instance, this space arises in compactness at the bending scale for the combined entropic and Frank energy studied in section 1.6. It does not appear that a result of this type has been considered so far. Our result for non-smooth midplane deformations satisfying $\tilde{\nabla} y \in \mathcal{D}_{r}$ a.e. is only stated for lifted surfaces, as these are the examples we can explicitly construct and approximate. 


\subsection{The metric constraint as a necessary condition for bending}

We come to our last main result. So far, we exhibited constructions (nonisometric origami and lifted surfaces) which satisfy the metric constraint (1.6) and this guarantees that they have small entropic strain energy $\left(O\left(h^{2}\right)\right.$ and $O\left(h^{3}\right)$ respectively).

The result we discuss now goes in the opposite direction. That is, we assume that the strain energy of a sequence of $Y^{h}$ (indexed by $h$ ) is of order $h^{3}$ (i.e. is small) and we prove that then the midplane part of $Y^{h}$ converges to a map $y: \omega \rightarrow \mathbb{R}^{3}$ satisfying the metric constraint.

This is a compactness result and as a technical ingredient it requires the Geometric Rigidity results of Friesecke, James and Müller [23]. To apply these in the present setting, we augment the original entropic elastic energy (1.1) with an additional term modeling (an approximation to) Frank elasticity. For this, we define the auxiliary map $N: \mathbb{R}^{3 \times 3} \times \mathbb{S}^{2} \rightarrow \mathbb{S}^{2} \cup\{+\infty\}$ as

$$
N\left(F, N_{0}\right)= \begin{cases}\frac{F N_{0}}{\left|F N_{0}\right|} & \text { if }\left|F N_{0}\right| \neq 0 \\ +\infty & \text { otherwise }\end{cases}
$$

As the elastic energy augmented by a Frank elastic term, we take the function $\mathcal{I}_{N_{0}^{h}}^{h, \varepsilon}$ given by

$$
\mathcal{I}_{N_{0}^{h}}^{h, \varepsilon}\left(Y^{h}\right):=\int_{\Omega_{h}}\left(\widehat{W}^{e}\left(\nabla Y^{h}, N\left(\nabla Y^{h}, N_{0}^{h}\right), N_{0}^{h}\right)+\varepsilon^{2}\left|\nabla N\left(\nabla Y^{h}, N_{0}^{h}\right)\right|^{2}\right) d x
$$

for an appropriate space of admissible deformations (compare $\mathcal{A}^{h}(\Omega)$ below). Here we introduced $\widehat{W}^{e}:=(\mu / 2)^{-1} W^{e}$, the dimensionless entropic energy density, and the lengthscale $\varepsilon>0$.

We come to the main result. For the statement, we find it convenient to rescale the $x_{3}$ variable so that we can work on the fixed domain $\Omega=\omega \times(-1 / 2,1 / 2)$. This yields

$$
\begin{array}{r}
W^{h}(z(x))=Y^{h}(x), \quad M_{0}^{h}(z(x))=N_{0}^{h}(x), \quad h^{-3} \mathcal{I}_{N_{0}^{h}}^{h, \varepsilon}\left(Y^{h}\right)=\mathcal{J}_{M_{0}^{h}}^{h, \varepsilon}\left(W^{h}\right) \\
\mathcal{J}_{M_{0}^{h}}^{h, \varepsilon}\left(W^{h}\right):=\int_{\Omega}\left(\frac{1}{h^{2}} \widehat{W}^{e}\left(\nabla_{h} W^{h}, N\left(\nabla_{h} W^{h}, M_{0}^{h}\right), M_{0}^{h}\right)+\frac{\varepsilon^{2}}{h^{2}}\left|\nabla_{h} N\left(\nabla_{h} W^{h}, M_{0}^{h}\right)\right|^{2}\right) d z
\end{array}
$$

where $N$ is defined as in (1.27). We consider this energy functional on the space of admissible rescaled deformations

$$
\mathcal{A}^{h}(\Omega):=\left\{W^{h} \in W^{1,2}\left(\Omega, \mathbb{R}^{3}\right): N\left(\nabla_{h} W^{h}, M_{0}^{h}\right) \in W^{1,2}\left(\Omega, \mathbb{S}^{2}\right)\right\} .
$$

Here, for $f: \Omega \rightarrow \mathbb{R}^{3}$, we denote $\nabla_{h} f$ as $\left(\tilde{\nabla} f \mid \frac{1}{h} \partial_{3} f\right)$, which reflects the rescaling of $x_{3}$ by $1 / h$.

Given these rescalings, we have

Theorem 1.12 (Compactness). Let $r>0$. Let $n_{0} \in W^{1,2}\left(\omega, \mathbb{S}^{2}\right)$ and let

$$
c_{l} h \leq \varepsilon \equiv \varepsilon_{h} \leq c_{u} h,
$$

for some constants $c_{u} \geq c_{l}>0$. Moreover, let $M_{0}^{h}$ satisfy

$$
M_{0}^{h}(z)=n_{0}(\tilde{z})+O(h), \quad \text { for a.e. } z \in \Omega, \quad \text { i.e., }\left\|M_{0}^{h}-n_{0}\right\|_{L^{\infty}(\Omega)} \leq \tau h .
$$

For every sequence $\left\{W^{h}\right\} \subset W^{1,2}\left(\Omega, \mathbb{R}^{3}\right)$ with $\mathcal{J}_{M_{0}^{h}}^{h, \varepsilon_{h}}\left(W^{h}\right) \leq C<\infty$ as $h \rightarrow 0$, there exists a subsequence (not relabeled) and a $y \in W^{2,2}\left(\Omega, \mathbb{R}^{3}\right)$ independent of $z_{3}$ such that as $h \rightarrow 0$

$$
\left(W^{h}-\frac{1}{|\Omega|} \int_{\Omega} W^{h} d z\right) \rightarrow y \quad \text { in } W^{1,2}\left(\Omega, \mathbb{R}^{3}\right) \quad \text { with } \quad(\tilde{\nabla} y)^{T} \tilde{\nabla} y=\tilde{\ell}_{n_{0}} \text { a.e. on } \omega \text {. }
$$


Remark 1.13. (i) Having control of the regularity $\nabla_{h} N\left(\nabla_{h} W^{h}, M_{0}^{h}\right)$ precisely with $\varepsilon$ of order $h$ is important to use Geometric Rigidity. In other words, we assume that Frank elasticity is comparable to entropic elasticity at the bending scale.

(ii) Let us discuss some background concerning Frank elasticity. Our augmented energy considers possible contributions due to Frank elasticity. Following de Gennes and Prost [20], Frank elasticity is a phenomenological continuum model for an energy penalizing distortions in the alignment of the current director $n$,

$$
W_{F r}=\frac{K_{1}}{2}(\operatorname{div} n)^{2}+\frac{K_{2}}{2}(n \cdot \operatorname{curl} n)^{2}+\frac{K_{3}}{2}|n \times \operatorname{curl} n|^{2} .
$$

Here, the three terms physically represent splay, twist and bend of the director field with respective moduli $K_{1}, K_{2}, K_{3}>0$. If the moduli are equal, i.e. $K_{i}=\kappa$ for $i=1,2,3$, then $W_{F r}$ reduces to

$$
W_{F r} \equiv \frac{\kappa}{2}|\operatorname{grad} n|^{2} .
$$

More generally, since the moduli $K_{i}$ are positive, we have the estimate

$$
\frac{1}{2} \kappa_{l}|\operatorname{grad} n|^{2} \leq W_{F r} \leq \frac{1}{2} \kappa_{u}|\operatorname{grad} n|^{2}
$$

for $\kappa_{l}=\min \left\{K_{1}, K_{2}, K_{3}\right\}$ and $\kappa_{u}=\max \left\{K_{1}, K_{2}, K_{3}\right\}$.

We are interested foremost in how Frank energy may compete with the entropic energy at the bending scale. Thus, we consider only the simplified model (1.34) since the detailed model is sandwiched energetically by models of this type (1.35). We make a further assumption regarding how distortions in nematic alignment are accounted in the energetic framework. To elaborate, a model for Frank elasticity should ideally penalize spatial distortions in the alignment of the director field, i.e., the div, curl and grad operators should be with respect to the current frame. Unfortunately, this seems quite technical to capture in a variational setting, as notions of invertibility of Sobolev maps must be carefully considered. It is, however, an active topic of mathematical research. For instance, we refer the interested reader to the works of Barchiesi and DeSimone [3] and Barchiesi et al. [4] for Frank elasticity and nematic elastomers in this context. Nevertheless, for our purpose in understanding whether the metric constraint (1.6) is necessitated by a smallness in the energy, we find it sufficiently interesting to consider the simplified model

$$
W_{F r} \approx \frac{\kappa}{2}\left|\nabla N^{h}\right|^{2}, \quad N^{h}: \Omega_{h} \rightarrow \mathbb{S}^{2}
$$

where $N^{h}$ refers to the current director field as a mapping from the initially flat sheet $\Omega_{h}$ and $\nabla$ is the gradient with respect to this reference state. The term (1.36) is the one that appears in the energy (1.28).

(iii) The parameter $\varepsilon=\sqrt{\kappa / \mu}$ is likely quite small in nematic elastomers. Specifically, in liquid crystal fluids, the moduli $K_{i}$ (which bound $\kappa$ ) have been measured in detail, and these moduli are likely similar for nematic elastomers (see, for instance, the discussion in Chapter 3 [43]). Further, the shear modulus $\mu$ of the rubbery network, which is distinct to elastomers, is much larger. Substituting the typical values for these parameters, we find $\varepsilon \sim 10-100 \mathrm{~nm}$. Thus, entropic elasticity will often dominate Frank elasticity in these elastomers. However, a typical 

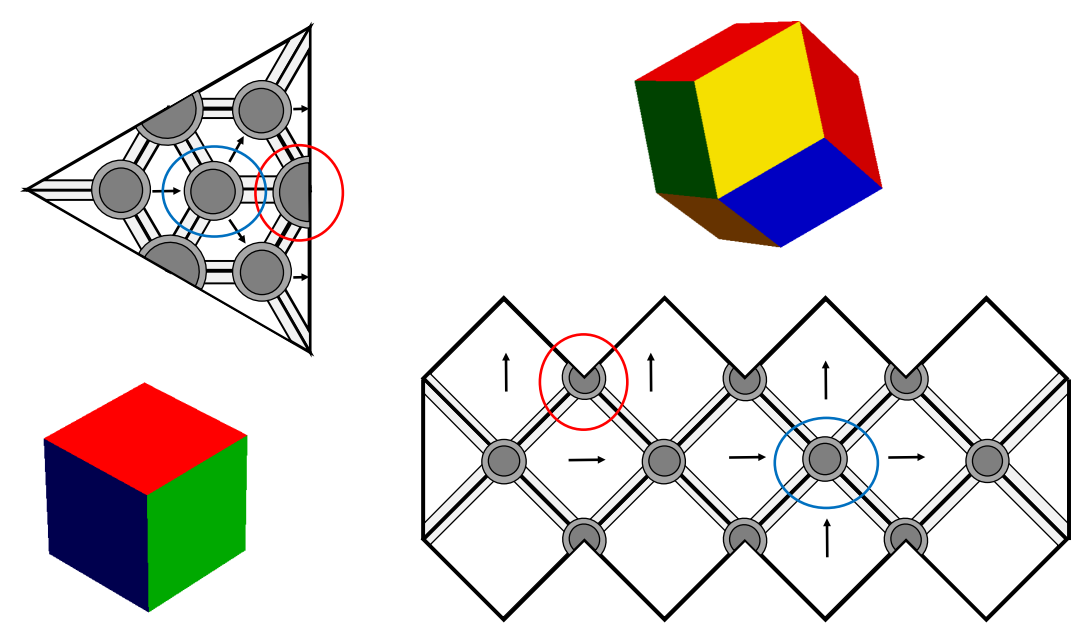

Figure 3: Sketch of smoothing procedure to approximate the Box and Rhombic Dodecahedron deformation. The interfaces are smoothed one dimensionally, while each junction is repaired in a disk or partial disk. For these symmetric constructions, there is a class of constructions suitable for all interior cases in blue and another class suitable for all the boundary cases in red.

thin sheet will have a thickness $h \sim 10-100 \mu \mathrm{m}$. So there are two small lengthscales to consider in this problem. For mechanical boundary conditions which induce stretch and stress in these sheets, the entropic energy does appear to dominate the Frank term. For instance, stripe domains of oscillating nematic orientation would be suppressed by a large Frank energy, and yet these have been observed by Kundler and Finkelmann [28] in the clamped stretch experiments on thin sheets. Mathematically, this dominance under stretch is made precise, for instance, by Cesana et al. [13] in studying an energy of type (1.28) for a general director $N$ and the resulting membrane theory does not depend on Frank elasticity. These results notwithstanding, actuation of nematic sheets with controlled heterogeneity occurs at a much lower energy state. Therefore, it is possible that the actuated configuration emerges from a non-trivial competition between entropic and Frank elasticity at these small energy scales. Hence, we study this competition in an asymptotic sense by taking $h$ and $\varepsilon \rightarrow 0$.

\section{Approximating the two-dimensional deformations for idealized actuation}

We now catalogue several results regarding the approximation of non-smooth two-dimensional deformations $y: \omega \rightarrow \mathbb{R}^{3}$ on a lengthscale $\delta>0$ via smoothings $y^{\delta}: \omega \rightarrow \mathbb{R}^{3}$. Each of these constructions is amenable to a three dimensional incompressible extension for small $h$ with $\delta=O(h)$. In Sections 2.1-2.3, we construct smooth approximations to junctions which form the unit cells of the nonisometric origami examples in Figure 13. These smoothings satisfy the approximation hypothesis (1.16), crucial to our energy argument. The idea is sketched in Figure 3. In section 2.4, we piece together these constructions to obtain global midplane deformations satisfying (1.16) for these examples of nonisometric origami. In addition, we construct an optimal vector associated to the out-of-plane deformation gradient at the midplane for any approximation to nonisometric origami in (1.16). Finally, in Section 2.5, we construct appropriate approximations to the lifted surface ansatz. 


\subsection{Smoothing with full-rank at each interface}

Suppose $\omega$ is the union of $K$ connected sectors about a junction $O$ with each sector $\omega_{\alpha}$ described by the angle $\theta_{\alpha}$. We assume $y: \omega \rightarrow \mathbb{R}^{3}$ is a continuous piecewise affine deformation satisfying

$$
y(\tilde{x})=\tilde{F}_{\alpha} \tilde{x} \quad \text { for } \quad \tilde{x} \in \omega_{\alpha}
$$

and such that each $\tilde{F}_{\alpha} \in \mathbb{R}^{3 \times 2}$ has $\operatorname{rank} \tilde{F}_{\alpha}=2$. Let each interface be described by the outward tangent $g_{\alpha} \in \mathbb{S}^{1}$ as in Figure 4 a with $g_{\alpha}^{\perp}$ its perpendicular as shown. We set $\tilde{x}:=x_{1} e_{1}+x_{2} e_{2}=$ $s_{\alpha} g_{\alpha}+t_{\alpha} g_{\alpha}^{\perp}$, and define $y_{\alpha}: \mathbb{R}^{2} \rightarrow \mathbb{R}^{3}$ given by

$$
y_{\alpha}\left(s_{\alpha}, t_{\alpha}\right)= \begin{cases}s_{\alpha} \tilde{F}_{\alpha} g_{\alpha}+t_{\alpha} \tilde{F}_{\alpha} g_{\alpha}^{\perp} & t_{\alpha} \leq 0 \\ s_{\alpha} \tilde{F}_{\alpha+1} g_{\alpha}+t_{\alpha} \tilde{F}_{\alpha+1} g_{\alpha}^{\perp} & t_{\alpha}>0 .\end{cases}
$$

If $\alpha=K$, then $\alpha+1=1$. Next, for the region $\omega_{g_{\alpha}} \subset \omega$ given by the sector of angle $\left(\theta_{\alpha}+\theta_{\alpha+1}\right) / 2$ as in the schematic in Figure $4 \mathrm{a}$, we define $y_{\alpha}^{\delta}: \omega_{g_{\alpha}} \rightarrow \mathbb{R}^{3}$ given by

$$
y_{\alpha}^{\delta}\left(s_{\alpha}, t_{\alpha}\right)=\int_{I_{\delta / 2}\left(t_{\alpha}\right)} \eta_{h}\left(t_{\alpha}-t\right) y_{\alpha}\left(s_{\alpha}, t\right) d t \quad \text { on } \omega_{g_{\alpha}}
$$

Here $I_{\delta / 2}\left(t_{\alpha}\right)=\left(-\delta / 2+t_{\alpha}, \delta / 2+t_{\alpha}\right)$, and $\eta_{\delta}$ is a standard symmetric mollifier with support on the interval $I_{\delta / 2}(0)$. Finally, we set $y_{0}^{\delta}: \omega \rightarrow \mathbb{R}^{3}$ as

$$
y_{0}^{\delta}= \begin{cases}y_{\alpha}^{\delta} & \text { on each } \omega_{g_{\alpha}} \\ y & \text { otherwise on } \omega .\end{cases}
$$

Note that the exceptional set occurs if $\Sigma_{\alpha=1}^{K} \theta_{\alpha}<2 \pi$, in which case the junction has a boundary. We make the following observations regarding this construction:

Proposition 2.1. Let $\theta_{0}:=\min \left\{\theta_{\alpha}: \alpha \in\{1, \ldots, K\}\right\}$, assume $\delta \ll 1$ and set $\rho_{0}^{\delta}:=\delta / \sin \left(\theta_{0} / 2\right)$. Let $\Gamma \subset \omega$ be the set containing all $g_{\alpha}$ interfaces and set $\Gamma_{\delta}:=\{\tilde{x} \in \omega: \operatorname{dist}(\tilde{x}, \Gamma)<\delta / 2\}$. If at each $g_{\alpha}$ interface

$$
\lambda \tilde{F}_{\alpha} g_{\alpha} \times \tilde{F}_{\alpha} g_{\alpha}^{\perp}+(1-\lambda) \tilde{F}_{\alpha+1} g_{\alpha} \times \tilde{F}_{\alpha+1} g_{\alpha}^{\perp} \neq 0 \quad \forall \lambda \in[0,1],
$$

then the restriction of $y_{0}^{\delta}$ to $\omega \backslash B_{\rho_{0}^{\delta}}(O)$ is in $C^{\infty}\left(\overline{\omega \backslash B_{\rho_{0}^{\delta}}(O)}, \mathbb{R}^{3}\right)$ and satisfies

$$
\left\|\partial_{1} y_{0}^{\delta} \times \partial_{2} y_{0}^{h}\right\|_{L^{\infty}} \geq c>0, \quad\left\|\tilde{\nabla} y_{0}^{\delta}\right\|_{L^{\infty}} \leq C, \quad\left\|\tilde{\nabla} \tilde{\nabla} y_{0}^{\delta}\right\|_{L^{\infty}} \leq C h^{-1}, \quad\left\|\tilde{\nabla}^{(3)} y_{0}^{\delta}\right\|_{L^{\infty}} \leq C \delta^{-2}
$$

on this set for some $c, C$ depending only on $y$. Further, $y_{0}^{\delta}$ has the representation

$$
\begin{aligned}
& y_{0}^{\delta}=y \quad \text { on } \omega \backslash\left(B_{\rho_{0}^{\delta}}(O) \cup \Gamma_{\delta}\right) \\
& y_{0}^{\delta}(\tilde{x})=s_{\alpha} \tilde{F}_{\alpha} g_{\alpha}+\gamma_{\delta}\left(t_{\alpha}\right) \tilde{F}_{\alpha} g_{\alpha}^{\perp}+\left(t_{\alpha}-\gamma_{\delta}\left(t_{\alpha}\right)\right) \tilde{F}_{\alpha+1} g_{\alpha}^{\perp} \quad \text { on each } \omega_{g_{\alpha}}
\end{aligned}
$$

with $\gamma_{\delta} \in C^{\infty}(\mathbb{R}, \mathbb{R})$ given by

$$
\gamma_{\delta}\left(t_{\alpha}\right):=\int_{I_{\delta / 2}\left(t_{\alpha}\right) \cap\{t \leq 0\}} \eta_{\delta}\left(t_{\alpha}-t\right) t d t
$$




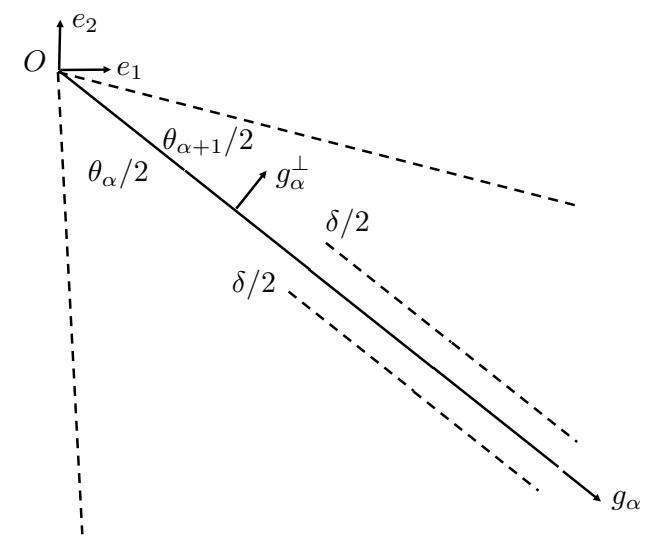

(a) Local cooridnate system for $\alpha$ interface

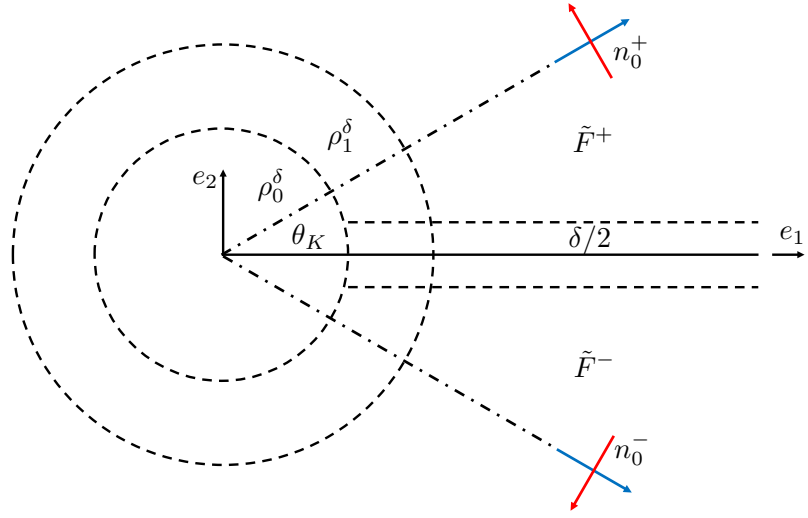

(b) Symmetric Junctions construction

Figure 4: Building blocks to smoothing the piecewise affine constructions

and satisfying

$$
\begin{aligned}
& \gamma_{\delta}\left(t_{\alpha}\right)= \begin{cases}t_{\alpha} & \text { for } t_{\alpha}<-\delta / 2 \\
0 & \text { for } t_{\alpha}>h / 2\end{cases} \\
& \gamma_{\delta}^{\prime}\left(t_{\alpha}\right)=\int_{I_{h / 2}\left(t_{\alpha}\right) \cap\{t \leq 0\}} \eta_{\delta}\left(t_{\alpha}-t\right) d t=: \lambda_{\delta}\left(t_{\alpha}\right)
\end{aligned}
$$

for $\lambda_{\delta} \in C^{\infty}(\mathbb{R},[0,1])$.

Before we prove this result, we note that if one is interested in actuating three dimensional shape that is idealized as a piecewise affine deformation from a flat sheet, then shapes which fold into themselves are unphysical. This is what is meant by the assumption in (2.4). If this assumption holds, then the proposition provides a construction for smoothing the piecewise affine deformation away from the junction $O$, and this smoothing has nice properties (2.5). If $\delta$ is small, then this smoothing is exactly the piecewise affine deformation desired for actuation outside a small region of measure $O(\delta)$ as shown in (2.6). The remaining representation formulas in the proposition will be useful later for our construction to repair the region around the junction.

Proof. To begin, observe that since by hypothesis $y$ is a continuous piecewise affine deformation, and each $g_{\alpha}$ interface is straight,

$$
\tilde{F}_{\alpha} g_{\alpha}=\tilde{F}_{\alpha+1} g_{\alpha}=: f_{\alpha} \quad \text { for each } g_{\alpha} .
$$

Thus given (2.1), $s_{\alpha} \mapsto y_{\alpha}\left(s_{\alpha}, t_{\alpha}\right)$ is smooth for $t_{\alpha}$ fixed. Hence, since $y_{\alpha}^{\delta}$ is a $\delta$-mollification of $y_{\alpha}$ in the direction for which $y_{\alpha}$ is not smooth but strictly Lipschitz continuous, we conclude $y_{\alpha}^{\delta} \in C^{\infty}\left(\mathbb{R}^{2}, \mathbb{R}^{3}\right)$ with

$$
\left\|\tilde{\nabla} y_{\alpha}^{\delta}\right\|_{L^{\infty}} \leq C \quad\left\|\tilde{\nabla} \tilde{\nabla} y_{\alpha}^{\delta}\right\|_{L^{\infty}} \leq C \delta^{-1} \quad\left\|\tilde{\nabla}^{(3)} y_{\alpha}^{\delta}\right\|_{L^{\infty}} \leq C \delta^{-2}
$$

where $C$ depends only on $y_{\alpha}$ and so $y$.

Now, combining (2.1), (2.2), (2.3) and (2.11), we obtain the local characterization of $y_{0}^{\delta}$ in $(2.7)$ using the fact that $\int_{\mathbb{R}} \eta_{\delta}(t) d t=1$. Thus on interior of each $\omega_{g_{\alpha}}$, we find that the gradient operator in the global frame is given by

$$
\tilde{\nabla} y_{0}^{\delta}(\tilde{x})=\left(\partial_{1} y_{\alpha}^{\delta} \mid \partial_{2} y_{\alpha}^{\delta}\right)(\tilde{x})=\left(\lambda_{\delta}\left(t_{\alpha}\right)\left(\tilde{F}_{\alpha} g_{\alpha} \mid \tilde{F}_{\alpha} g_{\alpha}^{\perp}\right)+\left(1-\lambda_{\delta}\left(t_{\alpha}\right)\right)\left(\tilde{F}_{\alpha+1} g_{\alpha} \mid \tilde{F}_{\alpha+1} g_{\alpha}^{\perp}\right)\right) \tilde{R}_{\alpha}
$$


where $\tilde{R}_{\alpha}:=e_{1} \otimes \tilde{\nabla} s_{\alpha}+e_{2} \otimes \tilde{\nabla} t_{\alpha}$ is a constant on $S O(2)$. Note that we have assumed in this calculation that $\gamma_{\delta}^{\prime}(t)=\lambda_{\delta}(t)$. This requires justification.

When $\delta / 2+t_{\alpha} \leq 0$, we find $I_{\delta / 2}\left(t_{\alpha}\right) \cap\{t \leq 0\}=I_{\delta / 2}\left(t_{\alpha}\right)$ and

$$
\gamma_{\delta}\left(t_{\alpha}\right)=\int_{I_{\delta / 2}\left(t_{\alpha}\right)} \eta_{\delta}\left(t_{\alpha}-t\right) t d t=\int_{-\delta / 2}^{\delta / 2} \eta_{\delta}(\tau)\left(t_{\alpha}-\tau\right) d \tau=t_{\alpha}
$$

since $\eta_{\delta}$ is a symmetric mollifier and $\tau$ is antisymmetric, and so there product vanishes upon this integration. Alternatively, if $-\delta / 2+t_{\alpha} \geq 0$, then $\left|I_{\delta / 2}\left(t_{\alpha}\right) \cap\{t \leq 0\}\right|=0$ and thus $\gamma_{\delta}\left(t_{\alpha}\right)=0$ in this case. These two results prove (2.9). For (2.10), we consider the case $-\delta / 2+t_{\alpha}<0<\delta / 2+t_{\alpha}$. In this case,

$$
\begin{aligned}
\gamma_{\delta}^{\prime}\left(t_{\alpha}\right) & =\int_{-\delta / 2+t_{\alpha}}^{0} \eta_{h}^{\prime}\left(t_{\alpha}-t\right) t d t=\int_{t_{\alpha}}^{\delta / 2} \eta_{\delta}^{\prime}(\tau)\left(t_{\alpha}-\tau\right) d \tau \\
& =-\eta_{\delta}\left(t_{\alpha}\right) t_{\alpha}-\int_{t_{\alpha}}^{h / 2}\left(\eta_{\delta}(\tau) \tau\right)^{\prime} d \tau-\int_{t_{\alpha}}^{\delta / 2} \eta_{\delta}(\tau) d \tau \\
& =\int_{-\delta / 2+t_{\alpha}}^{0} \eta_{\delta}\left(t_{\alpha}-t\right) d t=\lambda_{\delta}\left(t_{\alpha}\right)
\end{aligned}
$$

using various properties of the mollifier. In addition, given that the derivatives of the result in (2.9) coincide with $\lambda_{\delta}$ on these exceptional sets, we conclude (2.10) Thus, the formula in (2.13) is justified.

Now, we note that for $\tilde{G} \in \mathbb{R}^{3 \times 2}$ and $\tilde{R} \in S O(2),\left|\tilde{G} \tilde{R} e_{1} \times \tilde{G} \tilde{R} e_{2}\right|=\left|\tilde{G} e_{1} \times \tilde{G} e_{2}\right|$. Hence, combining this observation with (2.13), we find that on the interior of $\omega_{g_{\alpha}}$

$$
\begin{aligned}
\left|\partial_{1} y_{0}^{\delta} \times \partial_{2} y_{0}^{\delta}\right|(\tilde{x}) & =\left|f_{\alpha} \times\left(\lambda_{\delta}\left(t_{\alpha}\right) \tilde{F}_{\alpha} g_{\alpha}^{\perp}+\left(1-\lambda_{\delta}\left(t_{\alpha}\right)\right) \tilde{F}_{\alpha+1} g_{\alpha}^{\perp}\right)\right| \\
& =\left|\lambda_{\delta}\left(t_{\alpha}\right) f_{\alpha} \times \tilde{F}_{\alpha} g_{\alpha}^{\perp}+\left(1-\lambda_{\delta}\left(t_{\alpha}\right)\right) f_{\alpha} \times \tilde{F}_{\alpha+1} g_{\alpha}^{\perp}\right| \\
& \geq \inf _{\lambda \in[0,1]}\left|\lambda f_{\alpha} \times \tilde{F}_{\alpha} g_{\alpha}^{\perp}+(1-\lambda) f_{\alpha} \times \tilde{F}_{\alpha+1} g_{\alpha}^{\perp}\right| \geq c_{\alpha}>0
\end{aligned}
$$

where we have also used (2.11) and the fact that $\lambda_{\delta}$ maps to $[0,1]$. That this infimum is given by some $c_{\alpha}>0$ is a consequence of (2.4). Indeed, since $\tilde{F}_{\alpha}$ and $\tilde{F}_{\alpha+1}$ are full rank, we need only consider $\lambda \in(0,1)$. Then by $(2.4)$ and $(2.11), \lambda f_{\alpha} \times \tilde{F}_{\alpha} g_{\alpha} \neq(\lambda-1) f_{\alpha} \times \tilde{F}_{\alpha+1} g_{\alpha}$ for any $\lambda \in(0,1)$, and this result implies a $c_{\alpha}>0$.

We are now prepared to show that $y_{0}^{\delta}$ is smooth in $\omega \backslash B_{\rho_{0}^{\delta}}(O)$ and satisfies (2.5) and (2.6). For (2.6), note that by (2.7) and (2.9), we find $y_{0}^{\delta}=y$ on $\omega_{g_{\alpha}} \backslash \Gamma_{\delta}$. Thus by (2.3), we see that actually $y_{0}^{\delta}=y$ on $\omega \backslash \Gamma_{\delta}$. Now $\left(B_{\rho_{0}^{\delta}}(O) \cap \omega\right) \not \subset \Gamma_{\delta}$. This implies that on the radial boundaries of $\omega_{g_{\alpha}} \backslash B_{\rho_{0}^{\delta}}(O), y_{0}^{\delta}=y$. Therefore, since the piecewise affine $y$ has no jumps in its gradient on this set and since $y_{0}^{\delta}=y_{\alpha}^{\delta}$ on the interior of $\omega_{g_{\alpha}}$ with $y_{\alpha}^{\delta}$ smooth, we conclude that $y_{0}^{\delta}$ is smooth on $\omega \backslash B_{\rho_{0}^{\delta}}(O)$. Finally $y_{0}^{\delta}$ satisfies (2.6) since we have (2.12) and (2.14) and noting that the number of $g_{\alpha}$ interfaces is finite.

\subsection{Smoothing with full-rank in symmetric junctions: Interior Cases}

We now consider the smoothing of each of the junctions given by the symmetric problem described in Figure $4 \mathrm{~b}$. The difficulty here is to smooth so that the midplane deformation after smoothing is full-rank everywhere. The simplest case to consider is the interior junctions where for $K$ interfaces with $K \geq 3, \theta_{K}=\pi / K$ and the directors $n_{0}^{+}$and $n_{0}^{-}$are planar and given in blue in the figure. We 
have implicitly assumed the cooling case $r>1$ for this analysis. The exact analogue for heating can be obtained replacing the directors with their respective perpendiculars in the plane. In the cooling cases, we obtain a compatible pyramidal type deformation where in the global $\left\{e_{1}, e_{2}\right\}$ frame,

$$
\begin{aligned}
& y_{e_{1}}(\tilde{x}):=\left\{\begin{array}{cc}
\tilde{F}^{-} \tilde{x} & \text { for } \tilde{x} \in \omega_{e_{1}} \cap\left\{x_{2} \leq 0\right\} \\
\tilde{F}^{+} \tilde{x} & \text { for } \tilde{x} \in \omega_{e_{1}} \cap\left\{x_{2}>0\right\},
\end{array}\right. \\
& \tilde{F}^{ \pm}=r^{-1 / 6}\left(\begin{array}{cc}
1 & 0 \\
0 & 1 \\
\sqrt{r-1} \cos (\pi / K) & \pm \sqrt{r-1} \sin (\pi / K)
\end{array}\right)
\end{aligned}
$$

with $\omega_{e_{1}} \subset \omega$ the sector described in Figure $4 \mathrm{~b}$. Then the complete pyramidal piecewise affine midplane deformation $\bar{y}: \omega \rightarrow \mathbb{R}^{3}$ corresponding to this design is given by

$$
y(\tilde{x})=R_{K}^{\alpha} y_{e_{1}}\left(\left(\tilde{R}_{K}^{\alpha}\right)^{T} \tilde{x}\right) \quad \text { for } \tilde{x} \in \omega_{\tilde{R}_{K}^{\alpha} e_{1}}, \quad \alpha \in\{0, \ldots, K-1\} .
$$

Here $R_{K}^{\alpha}=R_{e_{3}}(2 \pi \alpha / K) \in S O(3)$ and $\tilde{R}_{K}^{\alpha}=\tilde{R}_{e_{3}}(2 \pi \alpha / K) \in S O(2)$ for

$$
R_{e_{3}}(\theta):=\left(\begin{array}{ccc}
\cos (\theta) & -\sin (\theta) & 0 \\
\sin (\theta) & \cos (\theta) & 0 \\
0 & 0 & 1
\end{array}\right), \quad \tilde{R}_{e_{3}}(\theta):=\left(\begin{array}{cc}
\cos (\theta) & -\sin (\theta) \\
\sin (\theta) & \cos (\theta)
\end{array}\right)
$$

and $\omega_{\tilde{R}_{K}^{\alpha} e_{1}}$ is the sector described by the $\tilde{R}_{K}^{\alpha}$ rotated $\omega_{e_{1}}$ depicted in Figure $4 \mathrm{~b}$.

This explicit construction characterizes the interior junctions circled in blue in Figure 3. Specifically, up to a rotation of the entire junction, the interior junctions for the box and rhombic dodecahedron are given explicitly by this result with $K=3$ and 4 respectively. To smooth out the junction and maintain full rank in these cases is relatively straightforward. The intuition is that the projection of these deformations onto the $\left\{e_{1}, e_{2}\right\}$ plane is a homogeneous contraction $r^{-1 / 6} I_{2 \times 2}$ as shown in Figure 5. This observation motivates a simple construction:

Proposition 2.2. Let $y$ as in (2.16) with $r>1$ and assume $\delta \ll 1$. Let $\Gamma \subset \omega$ be the set containing all the $\tilde{R}_{K}^{\alpha} e_{1}$ interfaces, and $\Gamma_{\delta}:=\{\tilde{x} \in \omega: \operatorname{dist}(\tilde{x}, \Gamma)<\delta / 2\}$. Let $\rho_{0}^{\delta}:=\delta / \sin (\pi / K)$ and $\rho_{1}^{\delta}:=\delta / \sin (\pi / K)+\delta / 2$ (see Figure $4 b$ ). There exists a $y^{\delta} \in C^{\infty}\left(\bar{\omega}, \mathbb{R}^{3}\right)$ such that

$$
\left\|\partial_{1} y^{\delta} \times \partial_{2} y^{\delta}\right\|_{L^{\infty}} \geq c>0, \quad\left\|\tilde{\nabla} y^{\delta}\right\|_{L^{\infty}} \leq C, \quad\left\|\tilde{\nabla} \tilde{\nabla} y^{\delta}\right\| \leq C \delta^{-1}, \quad\left\|\tilde{\nabla}^{(3)} y^{\delta}\right\|_{L^{\infty}} \leq C \delta^{-2}
$$

everywhere on $\omega$ for $c, C$ depending only on $y$. Moreover,

$$
y^{\delta}=y \quad \text { on } \omega \backslash\left(B_{\rho_{1}^{\delta}}(O) \cup \Gamma_{\delta}\right) .
$$

Proof. We apply Proposition 2.1 with $\theta_{0} / 2=\pi / K$ to obtain a $\bar{y}_{0}^{\delta}$ which has all the desired properties outside of the ball $B_{\rho_{0}^{\delta}}(O)$. We now modify this function in a superset of this bad set, the ball $B_{\rho_{1}^{\delta}}(O)$. Specifically, we define

$$
y^{\delta}=y_{0}^{\delta}+\psi_{\delta}\left(y_{c}-y_{0}^{\delta}\right)
$$

for $\psi_{\delta} \in C^{\infty}(\mathbb{R},[0,1])$ a smooth radial cutoff function $\psi_{\delta}=\psi_{\delta}(\rho)$ equal to 1 in $B_{\rho_{0}^{\delta}}(O)$ and 0 outside of $B_{\rho_{1}^{\delta}}(O)$. Thus, since the restriction of $y_{0}^{\delta}$ to $\omega \backslash B_{\rho_{0}^{\delta}}(O)$ belongs to $C^{\infty}\left(\overline{\omega \backslash B_{\rho_{0}^{\delta}}(O)}, \mathbb{R}^{3}\right)$, we see that $y^{\delta} \in C^{\infty}\left(\bar{\omega}, \mathbb{R}^{3}\right)$ so long as $y_{c} \in C^{\infty}\left(\bar{\omega}, \mathbb{R}^{3}\right)$. 


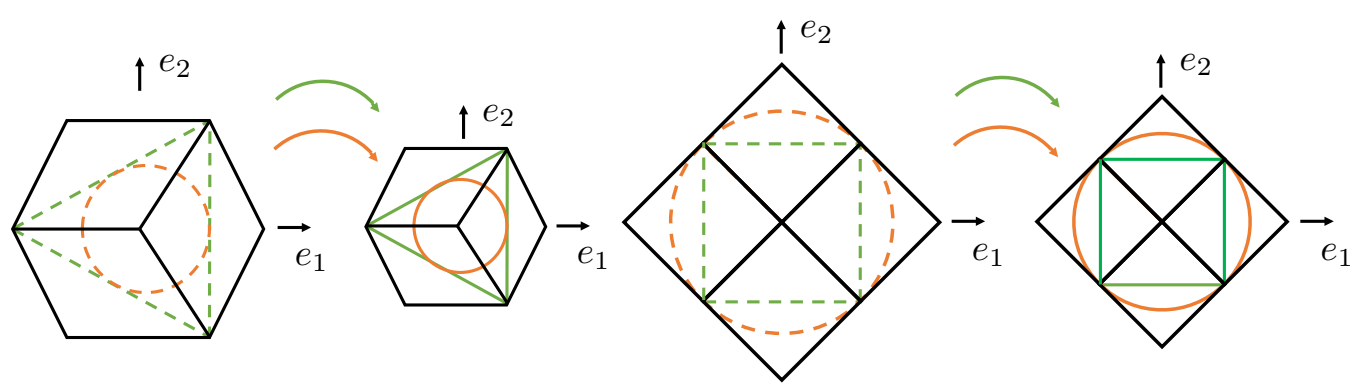

Figure 5: The interior junction for the box (left) and rhombic dodecahedron (right). The flat sheet becomes a pyramid, but in the projection shown, the dashed lines are uniformly contracted to the embolden lines.

Since the projection of $y$ in (2.16) to the $\left\{e_{1}, e_{2}\right\}$ plane is a homogeneous contraction $r^{-1 / 6}\left(x_{1} e_{1}+\right.$ $x_{2} e_{2}$ ) (see also Figure $4 \mathrm{~b}$ ), we take this as $y_{c}$, i.e.

$$
y_{c}=r^{-1 / 6} \rho e_{\rho}=r^{-1 / 6}\left(x_{1} e_{1}+x_{2} e_{2}\right) .
$$

Hence, $y^{\delta} \in C^{\infty}\left(\bar{\omega}, \mathbb{R}^{3}\right)$ and we have the equality in (2.19) by (2.6) in Proposition 2.1.

It remains to show the estimates in (2.18). These hold trivially in $B_{\rho_{0}^{\delta}}(O)$ since $y^{\delta}=y_{c}$ in this case. In fact, the bound on the gradients in this regime is independent of $h \ll 1$. Outside of $B_{\rho_{1}^{\delta}}(O)$, $y^{\delta}=y_{0}^{\delta}$ and these estimates follow from (2.5) in Proposition 2.1. Finally, on the punctured disk $B_{\rho_{1}^{\delta}}(O) \backslash B_{\rho_{0}^{\delta}}(O)$ (see Figure $4 \mathrm{~b}$ ), we compute

$$
\tilde{\nabla} y^{\delta}=\left(1-\psi_{\delta}\right) \tilde{\nabla} y_{0}^{\delta}+\psi_{\delta} \tilde{\nabla} y_{c}+\psi_{\delta}^{\prime}\left(y_{c}-y_{0}^{\delta}\right) \otimes e_{\rho} .
$$

We note that $\left|\psi_{\delta}^{\prime}\right| \leq C \delta^{-1}$ while $\left|y_{c}-y_{0}^{\delta}\right| \leq C \delta$, hence the third term above is at most $O(1)$. Thus, we conclude $\left|\tilde{\nabla} y^{\delta}\right| \leq C$ for $C$ depending only on $y$ since $\tilde{\nabla} y_{0}^{\delta}$ has this property (Proposition 2.1). Likewise, we find $\left|\tilde{\nabla} \tilde{\nabla} y^{\delta}\right| \leq C \delta^{-1}$ and $\left|\tilde{\nabla}^{(3)} y^{\delta}\right| \leq C \delta^{-2}$ by the chain rule since $y_{0}^{\delta}$ has this property and we may choose a cutoff function satisfying $\left|\psi_{\delta}^{\prime \prime}\right| \leq C \delta^{-2}$ and $\left|\psi_{\delta}^{(3)}\right| \leq C \delta^{-3}$ where each $C$ above is uniform or depends only on $y$.

For the cross-product bound, we denote $\tilde{y}^{\delta}: \omega \rightarrow \mathbb{R}^{2}$ as the projection of $y^{\delta}$ to the $\left\{e_{1}, e_{2}\right\}$ plane (and similarly for the other functions involved). Given this, we have $\left|\partial_{1} y^{\delta} \times \partial_{2} y^{\delta}\right| \geq\left|\operatorname{det}\left(\tilde{\nabla} \tilde{y}^{\delta}\right)\right|$, so we simply compute this determinant explicitly and show it does not vanish to prove the result. We note that in this calculation it suffices to consider $\tilde{y}^{\delta}$ restricted to $\omega_{e_{1}}$, i.e. $\tilde{y}_{e_{1}}^{\delta}$, as in Figure $4 \mathrm{~b}$ since it can be verified (akin to (2.16)) that

$$
\tilde{y}^{\delta}(\tilde{x})=\tilde{R}_{K}^{\alpha} \tilde{y}_{e_{1}}^{\delta}\left(\left(\tilde{R}_{K}^{\alpha}\right)^{T} \tilde{x}\right), \quad \tilde{x} \in \omega_{\tilde{R}_{K}^{\alpha} e_{1}}, \quad \alpha=\{0, \ldots, K-1\} .
$$

This leads to the identity $\operatorname{det}\left(\tilde{\nabla} \tilde{y}^{\delta}(\tilde{x})\right)=\operatorname{det}\left(\tilde{\nabla} \tilde{y}_{e_{1}}^{\delta}\left(\left(\tilde{R}_{K}^{\alpha}\right)^{T} \tilde{x}\right)\right)$, hence the sufficiency.

For this computation, we observe that $\tilde{F}_{2 \times 2}^{+}=\tilde{F}_{2 \times 2}^{-}=\bar{r}^{-1 / 6} I_{2 \times 2}$ where $\tilde{F}_{2 \times 2}^{ \pm} \in \mathbb{R}^{2 \times 2}$ is the $2 \times 2$ submatrix of $\tilde{F}^{ \pm}$in (2.15) associated to the projection $\tilde{y}$. Thus, following (2.7), we observe

$$
\tilde{y}_{0}^{\delta}(\tilde{x})=x_{1} \tilde{F}_{2 \times 2}^{-} e_{1}+\gamma_{\delta}\left(x_{2}\right) \tilde{F}_{2 \times 2}^{-} e_{2}+\left(x_{2}-\gamma_{\delta}\left(x_{2}\right)\right) \tilde{F}_{2 \times 2}^{+} e_{2}=r^{-1 / 6} I_{2 \times 2} \tilde{x} \quad \text { on } \omega_{e_{1}},
$$

and following $(2.20)$, we also have $\tilde{y}_{c}(\tilde{x})=r^{-1 / 6} I_{2 \times 2} \tilde{x}$ on $\omega$. Thus, we conclude

$$
\tilde{\nabla} \tilde{y}^{\delta}=\left(1-\psi_{\delta}\right) \tilde{\nabla} \tilde{y}_{0}^{\delta}+\psi_{\delta} \tilde{\nabla} \tilde{y}_{c}+\psi_{\delta}^{\prime}\left(\tilde{y}_{c}-\tilde{y}_{0}^{\delta}\right) \otimes e_{\rho}=r^{-1 / 6} I_{2 \times 2} \tilde{x} \quad \text { on } \omega_{e_{1}} .
$$

Evidently then, $\operatorname{det} \tilde{\nabla} \tilde{y}^{\delta}=r^{-1 / 3}$ everywhere on $\omega$, so the proof is complete. 

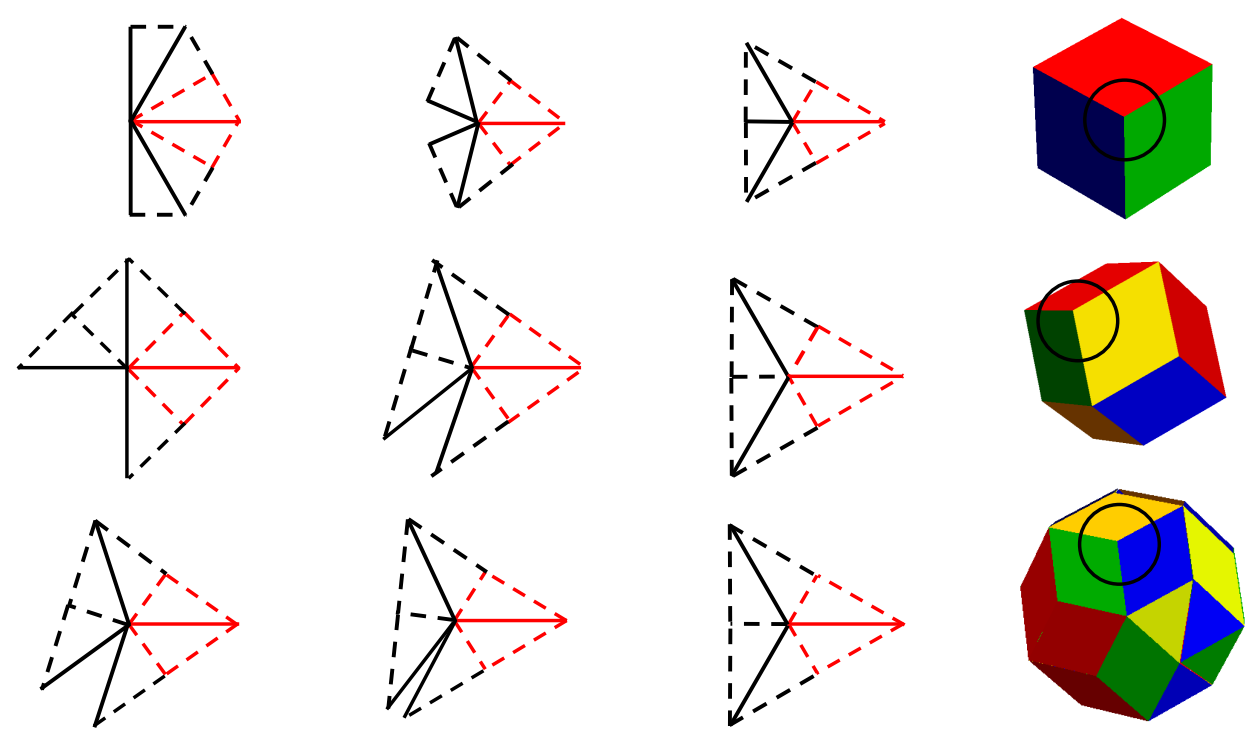

Figure 6: The unit cell in Figure 4b is emphasized in red. (Far Left) The initially undeformed flat boundary case. (Left) The projection of an intermediate deformation onto the $\left\{e_{1}, e_{2}\right\}$ plane. (Right) When the regions finally merge to form a complete corner at $r=3$ (box), $=2$ (rhombic dodecahedron $) \approx 1.38$ (rhombic triacontahedron).

\subsection{Smoothing with full-rank in symmetric junctions: Boundary Cases}

Next, we turn to the boundary cases characterized by the circled red regions in Figure 3. We can capture deformations where these boundaries eventually merge to form a regular polyhedra by careful consideration of the unit cell in Figure $4 \mathrm{~b}$ with the director now given in red. In these cases, we have the following description of the unit cell in the global $\left\{e_{1}, e_{2}\right\}$ frame:

$$
\begin{aligned}
& y_{e_{1}}(\tilde{x}):=\left\{\begin{array}{lll}
\tilde{F}^{-} \tilde{x} & \text { for } \tilde{x} \in \omega_{e_{1}} \cap\left\{x_{2} \leq 0\right\} \\
\tilde{F}^{+} \tilde{x} & \text { for } \tilde{x} \in \omega_{e_{1}} \cap\left\{x_{2}>0\right\},
\end{array}\right. \\
& \tilde{F}^{ \pm}=\frac{r^{5 / 6}}{c_{K}^{r}}\left(\begin{array}{cc}
1 & \mp \frac{(r-1)}{r \tan \left(\theta_{K}\right)} \\
\frac{c_{K}^{r}}{r} & \begin{array}{cc}
0 \\
\frac{\sqrt{r-1} \cos \left(\theta_{K}\right)}{r \tan \left(\theta_{K}\right)}
\end{array}
\end{array}\right) \\
& c_{K}^{r}:=\frac{\sqrt{r-\cos ^{2}\left(\theta_{K}\right)}}{\sin \left(\theta_{K}\right)}
\end{aligned}
$$

with $\omega_{e_{1}}$ the sector described in Figure $4 \mathrm{~b}$.

This unit cell captures the deformation at boundaries where a flat sheet merges to form a corner of the box, rhombic dodecahedron or rhombic triacontahedron. In particular, for these corners, we have

$$
\begin{array}{ll}
\theta_{K} \equiv \theta_{3}=\pi / 6: & y_{\text {box }}(\tilde{x})=Q_{3}^{\alpha} y_{e_{1}}\left(\left(\tilde{R}_{3}^{\alpha}\right)^{T} \tilde{x}\right), \quad \tilde{x} \in \omega_{\tilde{R}_{3}^{\alpha} e_{1}} \quad \alpha \in\{0, \pm 1\} \\
\theta_{K} \equiv \theta_{4}=\pi / 4: & y_{r d}(\tilde{x})= \begin{cases}Q_{4}^{\alpha} y_{e_{1}}\left(\left(\tilde{R}_{4}^{\alpha}\right)^{T} \tilde{x}\right), & \tilde{x} \in \omega_{\tilde{R}_{4}^{\alpha} e_{1}} \quad \alpha \in\{0,1\} \\
Q_{4}^{-1} y_{e_{1}}\left(\left(\tilde{R}_{4}^{-1}\right)^{T} \tilde{x}\right), & \tilde{x} \in \omega_{\tilde{R}_{4}^{-1} e_{1}}^{+} \\
Q_{4}^{2} y_{e_{1}}\left(\left(\tilde{R}_{4}^{2}\right)^{T} \tilde{x}\right), & \tilde{x} \in \omega_{\tilde{R}_{4}^{2} e_{1}}^{-}\end{cases}
\end{array}
$$




$$
\theta_{K} \equiv \theta_{5}=3 \pi / 10: \quad y_{r t}(\tilde{x})= \begin{cases}Q_{5}^{\alpha} y_{e_{1}}\left(\left(\tilde{R}_{5}^{\alpha}\right)^{T} \tilde{x}\right), & \tilde{x} \in \omega_{\tilde{R}_{5}^{\alpha} e_{1}} \quad \alpha \in\{0,1\} \\ Q_{5}^{-1} y_{e_{1}}\left(\left(\tilde{R}_{5}^{-1}\right)^{T} \tilde{x}\right), & \tilde{x} \in \omega_{\tilde{R}_{5}^{-1} e_{1}}^{+} \\ Q_{5}^{2} y_{e_{1}}\left(\left(\tilde{R}_{5}^{2}\right)^{T} \tilde{x}\right), & \tilde{x} \in \omega_{\tilde{R}_{5}^{2} e_{1}}^{-}\end{cases}
$$

for $y_{e_{1}}$ as in $(2.21)$ and $\tilde{F}^{ \pm}$as in $(2.22), \tilde{R}_{K}^{\alpha}=\tilde{R}_{e_{1}}\left(2 \alpha \theta_{K}\right)$ for $\tilde{R}_{e_{1}}$ in $(2.17)$, and $Q_{K}^{\alpha}=R_{e_{3}}\left(2 \alpha \tilde{\theta}_{K}\right)$ with

$$
\tilde{\theta}_{K}:=\arctan \left(c_{K}^{r} \tan \left(\theta_{K}\right)\right)
$$

and for $R_{e_{3}}$ in (2.17). In the diagram in Figure 6, we have plotted these deformations projected onto the $\left\{e_{1}, e_{2}\right\}$ plane. The key observations for these boundary cases is periodicity, and the fact that all these deformations have consistent behavior and this holds across all relevant values of $r \geq 1$. These observations allow us to focus exclusively on the unit cell in Figure 4b via a modification consistent across all cases to obtain a full rank smooth $\left(C^{3}(\bar{\omega})\right)$ junction, Figure 7 .

Proposition 2.3. Let $y \in\left\{y_{b o x}, y_{r d}, y_{r t}\right\}$, and let $r \in\left[1, r_{c}\right]$ where $r_{c}$ defines the deformation when the boundaries merge to form a corner $\left(r_{c}=3\right.$ (box), $=2$ (rhombic dodecahedron), $\approx 1.38$ (rhombic triacontahedron)). Assume $\delta \ll 1$, and let $\Gamma \subset \omega$ be the set containing all the $\tilde{R}_{K}^{\alpha} e_{1}$ interfaces and $\Gamma_{\delta}:=\{\tilde{x} \in \omega: \operatorname{dist}(\tilde{x}, \Gamma)<\delta / 2\}$. There exists a $\rho_{1}^{\delta}=M \delta$ and $a y^{\delta} \in C^{3}\left(\bar{\omega}, \mathbb{R}^{3}\right)$ such that

$$
y^{\delta}=y \quad \text { on } \omega \backslash\left(B_{\rho_{1}^{\delta}}(O) \cup \Gamma_{\delta}\right) .
$$

for some $M \geq 1 / \sin \left(\theta_{K}\right)$ depending only on $y$. Moreover, $y^{\delta}$ satisfies the estimates

$$
\left\|\partial_{1} y^{\delta} \times \partial_{2} y^{\delta}\right\|_{L^{\infty}} \geq c>0, \quad\left\|\tilde{\nabla} y^{\delta}\right\| \leq C, \quad\left\|\tilde{\nabla} \tilde{\nabla} y^{\delta}\right\|_{L^{\infty}} \leq C \delta^{-1}, \quad\left\|\tilde{\nabla}^{(3)} y^{\delta}\right\|_{L^{\infty}} \leq C \delta^{-2}
$$

for c, $C$ depending only on $y$.

We prove this proposition in three steps. In the first step, we repair $y$ on the ball $B_{\rho_{1}^{\delta}}(O)$ via the deformation described in Figure 7. This construction is good (i.e. satisfies (2.25) and (2.26)) outside of a neighborhood of the origin $O$. However, in this neighborhood its second and third derivative become unbounded. Thus in the second step, we modify the construction of the first step in the ball $B_{\delta / 2}(O)$ to obtain a construction in $C^{3}\left(\bar{\omega}, \mathbb{R}^{3}\right)$ which satisfies the upper bound estimates in (2.26). However, the rank of the gradient diminishes at the origin for this modification. Thus, in the final step, we make a small perturbation in the ball $B_{\rho_{1}^{\delta}}(O)$ which gives a full-rank gradient on all of $\omega$ and does not modify the gradient estimates established previously. The calculation is tedious, so we present the proof of this proposition in Appendix D.

\subsection{Smoothing of nonisometric origami}

Let $y: \omega \rightarrow \mathbb{R}^{3}$ such that $y \in\left\{y_{b o x}, y_{r d}, y_{r t}\right\}$ where the deformation is now a mapping from the entire domain $\omega$ as depicted with the box to the far left in Figure 8. We break up the domain $\omega$ into disjoint polygonal regions $\omega_{\beta}$ each containing a single junction and such that $\cup_{\beta} \omega_{\beta}=\omega$ as depicted with the dashed lines in the figure. We then translate and rotate our coordinate frame as necessary so that the deformation $y$ restricted to the region regions $\omega_{\beta}$ is captured by a deformation $y_{\beta}$ characterized by (2.15) and (2.16) or (2.22) and (2.23) up to a restriction of the reference domain in Figure $4 \mathrm{~b}$. This is depicted for the box in the middle/left part of Figure 8 where the red region highlights the sector captured in Figure $4 \mathrm{~b}$. We perform the appropriate smoothing of $y_{\beta}$ in this local frame, either with Proposition 2.2 or Proposition 2.3, to obtain a $y_{\beta}^{\delta}$ on $\omega_{\beta}$. This is depicted 

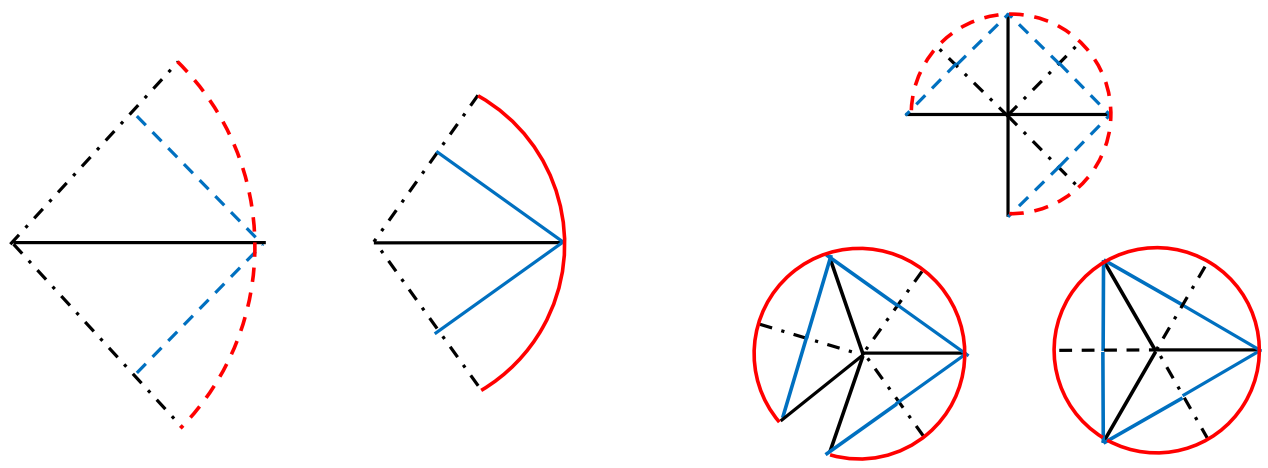

Figure 7: Unit cell (left) and examples of the global boundary deformation for rhombic dodecahedron (right). The dashed blue lines are deformed by the piecewise affine deformation to the blue lines. We correct the piecewise affine deformation employing a deformation (D.1) which takes the dashed red circular arcs to the red circular arcs. The affine deformation and correction are identical at each interface.
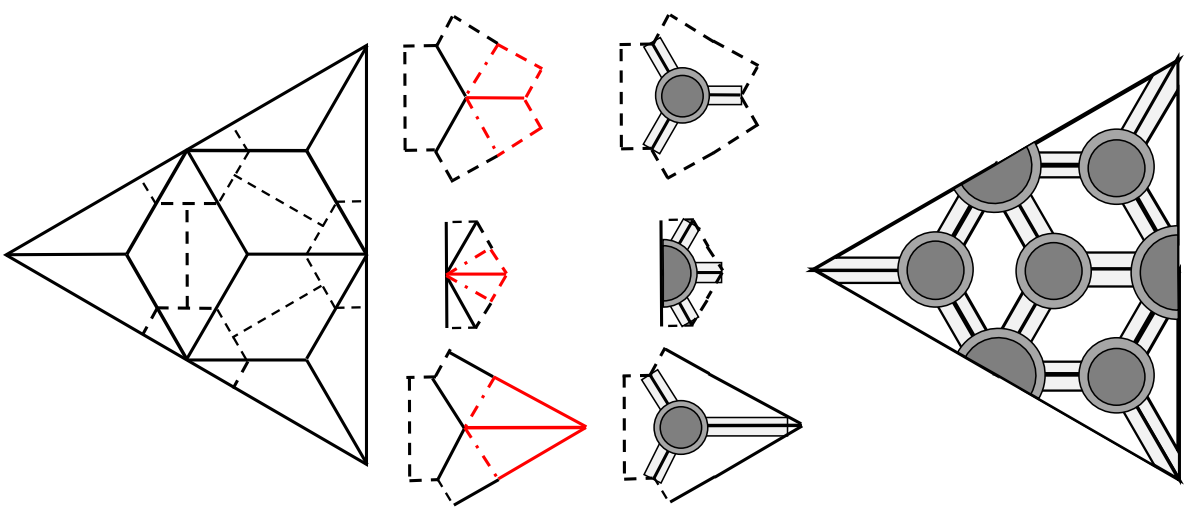

Figure 8: Smoothing of the box construction via local modifications

for the box in the middle/right part of Figure 8 where the shaded regions highlight our $\delta$-dependent smoothing. Finally, we translate and/or rotate our coordinate system as necessary back to the original frame. From this, we obtain a global deformation $y^{\delta}: \omega \rightarrow \mathbb{R}^{3}$ which is smooth across each $\partial \omega_{\beta} \cap \operatorname{int}(\omega)$ due to the compatibility of our constructions at these boundaries. This is depicted for the box on the far right in Figure 8.

Now, since each $y^{\delta}$ is obtained by the smoothing of junctions via Proposition 2.2 and 2.3, these globally smoothed midplane deformations actually satisfy the approximation hypothesis (1.16) for the appropriate $y \in\left\{y_{b o x}, y_{r d}, y_{r t}\right\}$. In addition, these are not the only nonisometric origami examples for which the explicit constructions in sections 2.1-2.3 are amenable. For instance, the examples in Figure 13c can be smoothed to obtain a global approximation $y^{\delta}$ satisfying (1.16) via application of Proposition 2.2 at each junction. Clearly then, there is not a dearth of examples privy to the analysis of the previous sections which leads to the approximation properties in (1.16). Hence, we assume (1.16) for our study of nonisometric origami in sequel.

We now construct a vector field $b^{\delta}: \omega \rightarrow \mathbb{R}^{3}$ that serves as the out-of-plane deformation gradient at the midplane $\omega$ which is consistent with a nearly zero energy midplane for any nonisometric origami in which the approximation property (1.16) holds:

Proposition 2.4. Let $r>0$ and $\delta \ll 1$. Let $\omega$ satisfy (1.9), $n_{0}$ satisfy (1.10), $y \in W^{1, \infty}\left(\omega, \mathbb{R}^{3}\right)$ 
satisfy (1.12) and each $\tilde{F}_{\alpha}$ and $n_{0 \alpha}$ in these relations satisfy (1.13). If there exists a $y^{\delta}$ satisfying (1.16), then for $\delta>0$ sufficiently small, there exists a $b^{\delta} \in C^{2}\left(\bar{\omega}, \mathbb{R}^{3}\right)$ such that

$$
\begin{aligned}
& \left(\tilde{\nabla} y^{\delta} \mid b^{\delta}\right)^{T}\left(\tilde{\nabla} y^{\delta} \mid b^{\delta}\right)=\ell_{n_{0}} \quad \text { and } \quad \tilde{\nabla} b^{\delta}=0 \quad \text { on } \omega \backslash \tilde{\omega}_{\delta} \quad \text { with }\left|\tilde{\omega}_{\delta}\right| \leq O(\delta), \\
& \operatorname{det}\left(\tilde{\nabla} y^{\delta} \mid b^{\delta}\right)=1 \quad \text { everywhere on } \omega .
\end{aligned}
$$

Moreover, $b^{\delta}$ satisfies

$$
\left\|b^{\delta}\right\|_{L^{\infty}} \leq C, \quad\left\|\tilde{\nabla} b^{\delta}\right\|_{L^{\infty}} \leq C \delta^{-1}, \quad\left\|\tilde{\nabla} \tilde{\nabla} b^{\delta}\right\|_{L^{\infty}} \leq C \delta^{-2}
$$

everywhere on $\omega$ for some $C>0$ which can depend on $y$ but is independent of $\delta$.

Proof. We define the vector valued function $b_{0}^{\delta}: \omega \rightarrow \mathbb{R}^{3}$ such that

$$
b_{0}^{\delta}:=\frac{\partial_{1} y^{\delta} \times \partial_{2} y^{\delta}}{\left|\partial_{1} y^{\delta} \times \partial_{2} y^{\delta}\right|^{2}}
$$

Given this definition and the properties of $y^{h}$ in $(1.16)$, we see that $b_{0}^{\delta} \in C^{2}\left(\bar{\omega}, \mathbb{R}^{3}\right)$ such that

$$
\begin{aligned}
& \left|b_{0}^{\delta}\right| \leq C, \quad\left|\tilde{\nabla} b_{0}^{\delta}\right| \leq C \delta^{-1}, \quad\left|\tilde{\nabla} \tilde{\nabla} b_{0}^{\delta}\right| \leq C \delta^{-2} \\
& \operatorname{det}\left(\tilde{\nabla} y^{\delta} \mid b_{0}^{\delta}\right)=\left(\partial_{1} y^{\delta} \times \partial_{2} y^{\delta}\right) \cdot b_{0}^{\delta}=1
\end{aligned}
$$

everywhere on $\omega$ and for some $C>0$ which depends on $y$ but is independent of $\delta$.

We will define $b^{\delta}$ as a modification of $b_{0}^{\delta}$. For this we note that the domain $\omega$ is composed of $N$ connected, disjoint polygonal regions $\omega_{\alpha}$ such the $\cup_{\alpha \in\{1, \ldots, N\}} \omega_{\alpha}=\omega$, e.g. the box example in Figure 8. We now focus on one particular $\omega_{\alpha}$ for the modification. Note that on this set, $y^{\delta}$ is modified from $y$ in a $O(\delta)$ neighborhood of $\partial \omega_{\alpha}$ only. Thus, for $\delta>0$ sufficiently small, we define $\tilde{\omega}_{\alpha}:=\bar{\gamma} \omega_{\alpha}+\bar{b} \subset \omega_{\alpha} \backslash \omega_{\delta}$ for some $\bar{\gamma} \in(0,1)$ and $\bar{b} \in \mathbb{R}^{3}$ with the following properties: Their exists a cutoff function $\psi_{\alpha}^{\delta} \in C_{0}^{\infty}\left(\omega_{\alpha},[0,1]\right)$ such that $\psi_{\alpha}^{\delta}=1$ on $\tilde{\omega}_{\alpha}, \psi_{\alpha}^{\delta}=0$ on $\omega_{\alpha} \cap \omega_{\delta}$, and

$$
\left|\tilde{\nabla} \psi_{\alpha}^{\delta}\right| \leq C \delta^{-1}, \quad\left|\tilde{\nabla} \tilde{\nabla} \psi_{\alpha}^{\delta}\right| \leq C h^{-2}
$$

on all of $\omega_{\alpha}$. Further, $\tilde{\omega}_{\alpha}$ is such that $\left|\omega_{\alpha} \backslash \tilde{\omega}_{\alpha}\right|=M_{\alpha} \delta$ for some constant $M_{\alpha}$ which may depend on $\omega_{\alpha}$ but is independent of $\delta \ll 1$.

For $\delta>0$ sufficiently small, we now fix each $\tilde{\omega}_{\alpha}$ and $\psi_{\alpha}^{\delta}$ for $\alpha=1, \ldots, N$ such that they have the properties described above. We observe that

$$
\tilde{\nabla} y^{\delta}=\tilde{\nabla} y=\tilde{F}_{\alpha} \quad \text { on each } \tilde{\omega}_{\alpha}
$$

where $\tilde{F}_{\alpha} \in \mathbb{R}^{3 \times 2}$ such that $\tilde{F}_{\alpha}^{T} \tilde{F}_{\alpha}=\tilde{\ell}_{n_{0 \alpha}}$ for $n_{0 \alpha} \in \mathbb{S}^{2}$ the uniform director associated to the director field $n_{0}: \omega \rightarrow \mathbb{S}^{2}$ in region $\omega_{\alpha}$. This follows directly from (1.12) and (1.16). Hence, by Proposition A.5, there exists a $b_{\alpha} \in \mathbb{R}^{3}$ such that

$$
\left(\tilde{F}_{\alpha} \mid b_{\alpha}\right)^{T}\left(\tilde{F}_{\alpha} \mid b_{\alpha}\right)=\ell_{n_{0 \alpha}}, \quad \operatorname{det}\left(\tilde{F}_{\alpha} \mid b_{\alpha}\right)=1 .
$$

Finally, we define $b^{\delta}: \omega \rightarrow \mathbb{R}^{3}$ such that

$$
b^{\delta}:=b_{0}^{\delta}+\psi_{\alpha}^{\delta}\left(b_{\alpha}-b_{0}^{\delta}\right) \quad \text { on each } \omega_{\alpha} .
$$

It remains to verify the desired identities (2.27), (2.28) and (2.29). For the first identity, we observe that $b^{\delta}=b_{\alpha}$ on each $\tilde{\omega}_{\alpha}$ by the definition of $\psi_{\alpha}^{h}$. Thus, it follows that $\left(\tilde{\nabla} y^{\delta} \mid b^{\delta}\right)^{T}\left(\tilde{\nabla} y^{\delta} \mid b^{\delta}\right)=$ 
$\left(\tilde{F}_{\alpha} \mid b_{\alpha}\right)^{T}\left(\tilde{F}_{\alpha} \mid b_{\alpha}\right)=\ell_{n_{0 \alpha}}$ and $\tilde{\nabla} b^{\delta}=0$ on each $\tilde{\omega}_{\alpha}$. Hence, $\left(\tilde{\nabla} y^{\delta} \mid b^{\delta}\right)^{T}\left(\tilde{\nabla} y^{\delta} \mid b^{\delta}\right)=\ell_{n_{0}}$ and $\tilde{\nabla} b^{\delta}=0$ on $\cup_{\alpha \in\{1, \ldots, N\}} \tilde{\omega}_{\alpha}$. Given this observation, we define $\tilde{\omega}_{\delta}:=\omega \backslash \cup_{\alpha \in\{1, \ldots, N\}} \tilde{\omega}_{\alpha}$. Further, we recall that $\tilde{\omega}_{\alpha}$ was defined such that $\left|\omega_{\alpha} \backslash \tilde{\omega}_{\alpha}\right|=O(\delta)$. Therefore, we conclude that $\left|\tilde{\omega}_{\delta}\right| \leq O(\delta)$ as desired, and the identity in (2.27) follows. For (2.28), we find

$$
\begin{aligned}
\operatorname{det}\left(\tilde{\nabla} y^{\delta} \mid b^{\delta}\right) & =\left(\partial_{1} y^{\delta} \times \partial_{2} y^{\delta}\right) \cdot b^{\delta} \\
& =\left(1-\psi_{\alpha}^{\delta}\right)\left(\partial_{1} y^{\delta} \times \partial_{2} y^{\delta}\right) \cdot b_{0}^{\delta}+\psi_{\alpha}^{\delta} \operatorname{det}\left(\tilde{F}_{\alpha} \mid b_{\alpha}\right)=1
\end{aligned}
$$

due to (2.31) and since $\tilde{\nabla} y^{\delta}=\tilde{F}_{\alpha}$ when $\psi_{\alpha}^{\delta}>0$. Finally, (2.29) follows from the chain rule given (2.32) and (2.33).

\subsection{Smoothing of lifted surfaces}

Let $r>1$. Let $n_{0}$ as in (1.22) and $y$ as in (1.20) for a graph $\varphi$ as in (1.21). Here $n_{0}$ can be a $W^{1, \infty}\left(\omega, \mathbb{S}^{2}\right)$ design and $y$ can be a $W^{2, \infty}\left(\omega, \mathbb{R}^{3}\right)$ depending on the regularity of $\varphi$, and our analysis requires smooth fields to extend the deformation $y$ to a three dimensional deformation. Thus, we approximate the fields by replacing $\varphi$ with a smooth $\delta$-dependent approximation $\varphi_{\delta}$. Specifically, we extended $\varphi$ to all of $\mathbb{R}^{2}$ yielding $\varphi \in W^{2, \infty}\left(\mathbb{R}^{2}, \mathbb{R}^{3}\right)$ (the extension is not relabeled), and we set

$$
\varphi_{\delta}:=\eta_{\delta} * \varphi \quad \text { on } r^{-1 / 6} \omega
$$

for a standard mollifier $\eta_{h}$ supported on a ball of radius $h / 2$. Thus:

Proposition 2.5. For $\delta>0$ sufficiently small, $\varphi_{\delta}$ in (2.34) belongs to $C_{0}^{\infty}\left(r^{-1 / 6} \omega, \mathbb{R}\right)$ and satisfies the estimates

$$
\begin{aligned}
& \left\|\varphi-\varphi_{\delta}\right\|_{W^{1, \infty}} \leq O(\delta), \quad\left\|\tilde{\nabla} \varphi_{\delta}\right\|_{L^{\infty}}<\lambda_{r}, \\
& \left\|\tilde{\nabla}^{(n)} \varphi_{\delta}\right\|_{L^{\infty}} \leq O\left(\delta^{2-n}\right), \quad \text { for any integer } n \geq 2 .
\end{aligned}
$$

Proof. $\varphi_{\delta}$ is smooth by mollification. It vanishes on the boundary of $r^{-1 / 6} \omega$ for $\delta>0$ sufficiently small since by (1.21), spt $\varphi \subset r^{-1 / 6} \omega_{m}:=r^{-1 / 6}\{\tilde{x} \in \omega: \operatorname{dist}(\tilde{x}, \omega)>m\}$ and since $\eta_{\delta}$ is supported on a ball of radius $\delta / 2$. From standard manipulation of the mollification (2.34), the estimate on the $W^{1, \infty}$ norm follows from the Lipschitz continuity of $\varphi$ and $\tilde{\nabla} \varphi$, the estimate on $\tilde{\nabla} \varphi_{h}$ follows from that fact that $\|\tilde{\nabla} \varphi\|_{L^{\infty}}<\lambda_{r}$ and the estimates on the higher derivatives follow from the fact that $\tilde{\nabla} \tilde{\nabla} \varphi \in L^{\infty}$.

Now, we define $n_{0}^{\delta}$ as $n_{0}$ in (1.22) and $y^{\delta}$ as $y$ in (1.20) each with the graph $\varphi$ replace by $\varphi_{\delta}$ in (2.34). We make the following observations:

Proposition 2.6. Let $\delta>0$ sufficiently small. Let $n_{0}^{\delta}$ and $y^{\delta}$ as defined above for $\varphi_{\delta}$ as in (2.34), $\varphi$ as in (1.21), $n_{0}$ as in (1.22) and $y$ as in (1.20). Then $n_{0}^{\delta} \in C^{\infty}\left(\bar{\omega}, \mathbb{S}^{2}\right)$ and $y^{\delta} \in C^{\infty}\left(\bar{\omega}, \mathbb{R}^{3}\right)$ and they satisfy

$$
\begin{aligned}
& \left(\tilde{\nabla} y^{\delta}\right)^{T}\left(\tilde{\nabla} y^{\delta}\right)=\tilde{\ell}_{n_{0}^{\delta}} \quad \text { on } \omega, \quad\left\|n_{0}^{\delta}-n_{0}\right\|_{L^{\infty}} \leq O(\delta), \quad\left\|y^{\delta}-y\right\|_{W^{1, \infty}} \leq O(\delta), \\
& \left\|\tilde{\nabla} y^{\delta}\right\|_{L^{\infty}}+\left\|\tilde{\nabla} \tilde{\nabla} y^{\delta}\right\|_{L^{\infty}}+\left\|\tilde{\nabla} n_{0}^{\delta}\right\|_{L^{\infty}} \leq C, \quad\left\|\tilde{\nabla}(3) y^{\delta}\right\|_{L^{\infty}}+\left\|\tilde{\nabla} \tilde{\nabla} n_{0}^{\delta}\right\|_{L^{\infty}} \leq C \delta^{-1}
\end{aligned}
$$

for $C$ independent of $\delta$. 
Proof. These properties are a consequence of the properties on $\varphi_{\delta}$ established in Proposition 2.5. In particular, smoothness follows since $\varphi_{\delta}$ is a mollification; the metric constraint holds by the equivalence (1.23) since $\left\|\tilde{\nabla} \varphi_{\delta}\right\|_{L^{\infty}}<\lambda_{r}$; the estimates on the approximations $n_{0}^{\delta}-n_{0}$ and $y^{\delta}-y$ follow from the $W^{1, \infty}$ estimate of $\varphi_{\delta}-\varphi$ using the explicit definition of each field; and the $\delta$ dependent derivative estimates follow from the $\delta$-dependent derivative estimates of $\varphi_{\delta}$ again using the explicit definition of each field.

Now, for the midplane fields approximating the lifted surface ansatz, it remains to construct the out-of-plane vector $b^{\delta}: \omega \rightarrow \mathbb{R}^{3}$ approximating the out-of-plane deformation gradient at the midplane. To this end, we have:

Proposition 2.7. Let $\delta>0$ sufficiently small. Let $n_{0}^{\delta}$ and $y^{\delta}$ as in Proposition 2.6. There exists a $b^{\delta} \in C^{\infty}\left(\bar{\omega}, \mathbb{R}^{3}\right)$ such that

$$
\begin{aligned}
& \left(\tilde{\nabla} y^{\delta} \mid b^{\delta}\right)^{T}\left(\tilde{\nabla} y^{\delta} \mid b^{\delta}\right)=\ell_{n_{0}^{\delta}}, \quad \operatorname{det}\left(\tilde{\nabla} y^{h} \mid b^{h}\right)=1, \\
& \left\|b^{\delta}\right\|_{L^{\infty}}+\left\|\tilde{\nabla} b^{\delta}\right\|_{L^{\infty}} \leq C, \quad\left\|\tilde{\nabla} \tilde{\nabla} b^{\delta}\right\|_{L^{\infty}} \leq C \delta^{-1}
\end{aligned}
$$

for $C$ independent of $\delta$.

Proof. Since by Proposition 2.6, we have $\left(\tilde{\nabla} y^{\delta}\right)^{T} \tilde{\nabla} y^{\delta}=\tilde{\ell}_{n_{0}^{\delta}}$ everywhere on $\omega$, we apply Proposition A.5 pointwise everywhere on $\omega$. Thus, we define the vector $b^{\delta}: \omega \rightarrow \mathbb{R}^{3}$ as in (A.5) with $\tilde{\nabla} y^{\delta}$ replacing $\tilde{F}$ and $n_{0}^{\delta}$ replacing $n_{0}$ in these relations. Hence, (2.35) holds on $\omega$. Smoothness follows since $n_{0}^{\delta}, y^{\delta}$ and the parameterization (A.5) are each themselves smooth. The estimates on the derivatives of $b^{\delta}$ follow from the estimates on the derivative of $y^{\delta}$ and $n_{0}^{\delta}$ in Proposition 2.6 by explicit differentiation of the parameterization in (A.5).

\section{Incompressibility and the energy of three dimensional constructions}

In this section, for $h>0$ sufficiently small we construct deformations $Y^{h}: \Omega_{h} \rightarrow \mathbb{R}^{3}$ which approximate nonisometric origami, lifted surfaces and sufficiently smooth surfaces. We show that each of these constructions has a nontrivial $h$-dependence entropic energy scaling. In Section 3.1, we take the smooth approximations in Section 2, and extend them through the thickness to an incompressible global deformation. Next in Section 3.2, we define each of the constructions approximating the desired actuation. Finally, we prove the thickness scaling of the entropic energy: Theorem 1.4 for nonisometric origami is proved in Section 3.3, and Theorem 1.8 for lifted surfaces and Corollary 1.9 for sufficiently smooth surfaces are proved in Section 3.4.

\subsection{Incompressibility of thin elastomers}

We begin by using the approximations developed in Section 2 to construct incompressible three dimensional deformations for $h>0$ sufficiently small. The lemma below contains, by hypothesis, the essential features to these approximations which allow for incompressible extensions. This is parameterized by $\alpha \in\{-1,0,1\}$. $\alpha=1$ corresponds to nonisometric origami, $\alpha=0$ corresponds to lifted surfaces, and $\alpha=-1$ corresponds to smooth surfaces.

Lemma 3.1. Let $\alpha \in\{-1,0,1\}$. Suppose for any $0<\delta \ll 1$ we have $y_{\alpha}^{\delta} \in C^{3}\left(\bar{\omega}, \mathbb{R}^{3}\right)$ and $b_{\alpha}^{\delta} \in C^{2}\left(\bar{\omega}, \mathbb{R}^{3}\right)$ satisfying

$$
\operatorname{det}\left(\tilde{\nabla} y_{\alpha}^{\delta} \mid b_{\alpha}^{\delta}\right)=1 \quad \text { on } \omega
$$




$$
\begin{aligned}
& \left\|\tilde{\nabla} y_{\alpha}^{\delta}\right\|_{L^{\infty}(\omega)}+\left\|b_{\alpha}^{\delta}\right\|_{L^{\infty}(\omega)} \leq M, \\
& \left\|\tilde{\nabla} \tilde{\nabla} y_{\alpha}^{\delta}\right\|_{L^{\infty}(\omega)}+\left\|\tilde{\nabla} b_{\alpha}^{\delta}\right\|_{L^{\infty}(\omega)} \leq M \delta^{\min \{-\alpha, 0\}}, \\
& \left\|\tilde{\nabla}{ }^{(3)} y_{\alpha}^{\delta}\right\|_{L^{\infty}(\omega)}+\left\|\tilde{\nabla} \tilde{\nabla} b_{\alpha}^{\delta}\right\|_{L^{\infty}(\omega)} \leq M \delta^{-\alpha-1}
\end{aligned}
$$

for some uniform constant $M>0$. Then there exists an $m \equiv m(M, \alpha) \geq 1$ such that for any $h>0$ sufficiently small, there exists a $\xi_{\alpha}^{h} \in C^{1}\left(\bar{\Omega}_{h}, \mathbb{R}\right)$ and an extension $Y_{\alpha}^{h} \in C^{1}\left(\bar{\Omega}_{h}, \mathbb{R}^{3}\right)$ satisfying

$$
Y_{\alpha}^{h}=y_{\alpha}^{\delta_{h}}+\xi_{\alpha}^{h} b_{\alpha}^{\delta_{h}}, \quad \text { with } \quad \delta_{h}=m h \quad \text { and } \quad \operatorname{det} \nabla Y_{\alpha}^{h}=1 \quad \text { on } \Omega_{h} .
$$

In additions, $\xi_{\alpha}^{h}$ satisfies the pointwise estimates

$$
\left|\xi_{\alpha}^{h}-x_{3}\right| \leq C h^{\min \{-\alpha, 0\}}\left|x_{3}\right|^{2}, \quad\left|\partial_{3} \xi_{\alpha}^{h}-1\right| \leq C h^{\min \{-\alpha, 0\}}\left|x_{3}\right|, \quad\left|\tilde{\nabla} \xi_{\alpha}^{h}\right| \leq C h^{-\alpha-1}\left|x_{3}\right|^{2} .
$$

everywhere on $\Omega_{h}$. Here, each $C \equiv C(M)$ and does not depend on $h$.

Proof. We set $\delta_{h}=m h$ for $m \geq 1$ to be determined in Proposition 3.2. We consider the function

$$
V_{\alpha}^{h}\left(\tilde{x}, x_{3}\right):=y_{\alpha}^{\delta_{h}}(\tilde{x})+x_{3} b_{\alpha}^{\delta_{h}}(\tilde{x})
$$

and assume $x_{3} \in(-h / 2, h / 2)$. Since $\nabla V_{\alpha}^{h}=\left(\tilde{\nabla} y_{\alpha}^{\delta_{h}} \mid b_{\alpha}^{\delta_{h}}\right)+x_{3}\left(\tilde{\nabla} b_{\alpha}^{\delta_{h}} \mid 0\right)$ and $\operatorname{det}\left(\tilde{\nabla} y_{\alpha}^{\delta_{h}} \mid b_{\alpha}^{\delta_{h}}\right)=1$, we let $S_{\alpha}^{h}:=\left(\tilde{\nabla} y_{\alpha}^{\delta_{h}} \mid b_{\alpha}^{\delta_{h}}\right)^{-1}\left(\tilde{\nabla} b_{\alpha}^{\delta_{h}} \mid 0\right)$ and find

$$
\begin{aligned}
\operatorname{det} \nabla V_{\alpha}^{h} & =\operatorname{det}\left(\left(\tilde{\nabla} y_{\alpha}^{\delta_{h}} \mid b_{\alpha}^{\delta_{h}}\right)^{-1} \nabla V_{\alpha}^{h}\right)=\operatorname{det}\left(I+x_{3} S_{\alpha}^{h}\right) \\
& =1+x_{3} \operatorname{Tr}\left(S_{\alpha}^{h}\right)+x_{3}^{2} \operatorname{Tr}\left(\operatorname{cof} S_{\alpha}^{h}\right)+x_{3}^{3} \operatorname{det}\left(S_{\alpha}^{h}\right) .
\end{aligned}
$$

For the estimates below, $C \equiv C(M)$. We note that $\left\|\left(\tilde{\nabla} y^{\delta_{h}} \mid b^{\delta_{h}}\right)^{-1}\right\|_{L^{\infty}(\omega)} \leq C$ since the determinant is unity, and therefore $\left|S_{\alpha}^{h}\right| \leq C \delta_{h}^{\min \{-\alpha, 0\}}$ by hypothesis and

$$
\begin{aligned}
\left|\operatorname{det} \nabla V_{\alpha}^{h}-1\right| & \leq C\left(\left|x_{3}\right| \delta_{h}^{\min \{-\alpha, 0\}}+\left|x_{3}\right|^{2} \delta_{h}^{2 \min \{-\alpha, 0\}}+\left|x_{3}\right|^{3} \delta_{h}^{3 \min \{-\alpha, 0\}}\right) \\
& \leq C\left|x_{3}\right| \delta_{h}^{\min \{-\alpha, 0\}} \quad \text { for } \quad \alpha \in\{-1,0,1\}, \quad m \geq 1
\end{aligned}
$$

In addition for $\beta=1,2$, since $\left\|\partial_{\beta} S_{\alpha}^{h}\right\|_{L^{\infty}(\omega)} \leq C\left(\delta_{h}^{2 \min \{-\alpha, 0\}}+\delta_{h}^{-\alpha-1}\right) \leq C \delta_{h}^{-\alpha-1}$ for $\alpha \in\{-1,0,1\}$ and since $\left|\partial_{\beta} \operatorname{Tr}\left(S_{\alpha}^{h}\right)\right| \leq\left|\partial_{\beta} S_{\alpha}^{h}\right|,\left|\partial_{\beta} \operatorname{Tr}\left(\operatorname{cof} S_{\alpha}^{h}\right)\right| \leq 2\left|S_{\alpha}^{h}\right|\left|\partial_{\beta} S_{\alpha}^{h}\right|$ and $\left|\partial_{\beta} \operatorname{det}\left(S_{\alpha}^{h}\right)\right| \leq\left|S_{\alpha}^{h}\right|^{2}\left|\partial_{\beta} S_{\alpha}^{h}\right|$, we conclude that

$$
\begin{aligned}
\left|\partial_{\beta} \operatorname{det} \nabla V_{\alpha}^{h}\right| & \leq C\left(\left|x_{3}\right| \delta_{h}^{-\alpha-1}+\left|x_{3}\right|^{2} \delta_{h}^{\min \{-\alpha, 0\}} \delta_{h}^{-\alpha-1}+\left|x_{3}\right|^{3} \delta_{h}^{2 \min \{-\alpha, 0\}} \delta_{h}^{-\alpha-1}\right) \\
& \leq C\left|x_{3}\right| \delta_{h}^{-\alpha-1} \quad \text { for } \beta \in\{1,2\}, \quad \alpha \in\{-1,0,1\}, \quad m \geq 1 .
\end{aligned}
$$

Now since $V_{\alpha}^{h}$ is not incompressible, we modify it through a non-linear change in coordinates. We let $\Xi^{h}\left(\tilde{x}, x_{3}\right)=\left(\tilde{x}, \xi^{h}\left(\tilde{x}, x_{3}\right)\right)$ for $\xi^{h} \in C^{1}\left(\bar{\Omega}_{h}, \mathbb{R}\right)$ to be determined, and we define $Y_{\alpha}^{h}:=V_{\alpha}^{h} \circ \Xi^{h}$. Hence, by the column linearity of the determinant, we find that

$$
\operatorname{det} \nabla Y_{\alpha}^{h}=\operatorname{det}\left(\nabla V_{\alpha}^{h} \circ \Xi^{h}\right) \partial_{3} \xi^{h} .
$$

Thus, satisfying the determinant constraint on $\nabla Y^{h}$ amounts to satisfying the ordinary differential equation

$$
\partial_{3} \xi^{h}=\frac{1}{\operatorname{det}\left(\nabla V_{\alpha}^{h} \circ \Xi^{h}\right)} \quad \text { on } \quad \Omega_{h}
$$


for some $\xi^{h}$. There is an $m=m(\alpha, M) \geq 1$ such that for $h>0$ sufficiently small, there is a solution to $(3.7)$, i.e. $\xi^{h} \equiv \xi_{\alpha}^{h}$ for a $\xi_{\alpha}^{h} \in C^{1}\left(\bar{\Omega}_{h}, \mathbb{R}\right)$ with the initial condition $\xi_{\alpha}^{h}(\tilde{x}, 0)=0$, see Proposition 3.2 .

It remains to prove the estimates in (3.2). By Proposition 3.2, the map $\xi_{\alpha}^{h}$ satisfies pointwise

$$
\left|\xi_{\alpha}^{h}\right| \leq 2\left|x_{3}\right|, \quad\left|\partial_{3} \xi_{\alpha}^{h}\right| \leq 2
$$

everywhere on $\Omega_{h}$. Thus, given (3.7),(3.5) and the estimates above, we deduce

$$
\left|\partial_{3} \xi_{\alpha}^{h}-1\right| \leq\left|\partial_{3} \xi_{\alpha}^{h}\right|\left|\operatorname{det}\left(\nabla V^{h} \circ \Xi_{\alpha}^{h}\right)-1\right| \leq C h^{\min \{-\alpha, 0\}}\left|\xi_{\alpha}^{h}\right| \leq C h^{\min \{-\alpha, 0\}}\left|x_{3}\right|
$$

everywhere on $\Omega_{h}$. Similarly,

$$
\left|\xi_{\alpha}^{h}-x_{3}\right| \leq\left|\int_{0}^{x_{3}}\left(\partial_{3} \xi_{\alpha}^{h}-1\right) d \bar{x}_{3}\right| \leq \int_{0}^{\left|x_{3}\right|}\left|\partial_{3} \xi_{\alpha}^{h}-1\right| d \bar{x}_{3} \leq C h^{\min \{-\alpha, 0\}}\left|x_{3}\right|^{2}
$$

everywhere on $\Omega_{h}$. Finally, to estimate the first and second derivative of $\xi_{h}$, we define $F_{h}(\tilde{x}, t):=$ $\int_{0}^{s} \operatorname{det}\left(\nabla V^{h}(\tilde{x}, s)\right) d s$, and notice that the ordinary differential equation in (3.7) is equivalent to the implicit equation $F_{h}\left(\tilde{x}, \xi_{h}(x)\right)=x_{3}$. Differentiating this equation with respect to $x_{\beta}$, $\beta=1$, or 2 , we find

$$
\int_{0}^{\xi_{\alpha}^{h}} \partial_{\beta} \operatorname{det}\left(\nabla V^{h}\right) d s+\operatorname{det}\left(\nabla V^{h} \circ \Xi_{\alpha}^{h}\right) \partial_{\beta} \xi_{\alpha}^{h}=0 .
$$

Hence using (3.7), (3.6) and (3.8),

$$
\left|\partial_{\beta} \xi_{\alpha}^{h}\right| \leq\left|\partial_{3} \xi_{\alpha}^{h}\right| \int_{0}^{\left|\xi_{h}\right|}\left|\partial_{\beta} \operatorname{det} \nabla V^{h}\right| d s \leq C h^{-\alpha-1} \int_{0}^{\left|\xi_{\alpha}^{h}\right|}|s| d s \leq C h^{-\alpha-1}\left|x_{3}\right|^{2}
$$

everywhere on $\Omega_{h}$ for $\beta=1,2$. These are the desired estimates.

Proposition 3.2. Let $\alpha \in\{-1,0,1\}$. Let $V_{\alpha}^{h}$ defined in (3.3) with $y_{\alpha}^{\delta_{h}}$ and $b_{\alpha}^{\delta_{h}}$ as in Lemma 3.1 with $\delta_{h}=m h$. There is an $m=m(\alpha, M) \geq 1$ such that for any $h>0$ sufficiently small, there exists $a \xi_{\alpha}^{h} \in C^{\infty}\left(\bar{\Omega}_{h}, \mathbb{R}\right)$ such that

$$
\partial_{3} \xi_{\alpha}^{h}=\frac{1}{\operatorname{det}\left(\nabla V^{h} \circ \Xi^{h}\right)} \quad \text { on } \Omega_{h_{0}}, \quad \text { with } \quad \xi_{\alpha}^{h}(\tilde{x}, 0)=0 .
$$

Moreover $\xi_{\alpha}^{h}$ satisfies pointwise the estimate

$$
\left|\xi_{\alpha}^{h}\right| \leq 2\left|x_{3}\right|, \quad\left|\partial_{3} \xi_{\alpha}^{h}\right| \leq 2 \quad \text { on } \Omega_{h} .
$$

Remark 3.3. We can choose $m=1$ for $\alpha=\{-1,0\}$. For $\alpha=1$, we generally have to choose $m$ such that $m \geq \max \{C(M), 1\}$ where $C(M)$ is a constant that depends on $M$ but is independent of $h$.

Proof. For $\alpha \in\{-1,0,1\}$ and $h>0$, we consider the mapping $T_{\alpha}^{h}: \mathcal{M}_{\alpha}^{h} \rightarrow C\left(\bar{\Omega}_{h}\right)$ given by

$$
T_{\alpha}^{h}(\phi)\left(\tilde{x}, x_{3}\right)=\int_{0}^{x_{3}} \frac{1}{\operatorname{det}\left(\nabla V_{\alpha}^{h}(\tilde{x}, \phi(\tilde{x}, s))\right)} d s \quad \text { for each } \quad\left(\tilde{x}, x_{3}\right) \in \Omega_{h},
$$

where $\mathcal{M}_{\alpha}^{h}$ is given by

$$
\mathcal{M}_{\alpha}^{h}:=\left\{\phi \in C\left(\bar{\Omega}_{h}\right): \phi(\tilde{x}, 0)=0, \quad\left|\phi\left(\tilde{x}, x_{3}\right)\right| \leq 2\left|x_{3}\right|,\right.
$$




$$
\left.\operatorname{det}\left(\nabla V_{\alpha}^{h}\left(\tilde{x}, \phi\left(\tilde{x}, x_{3}\right)\right)\right) \geq 1 / 2 \text { for each }\left(\tilde{x}, x_{3}\right) \in \Omega_{h}\right\}
$$

This is a (non-empty) complete space under the infinity norm. Thus, we aim to show that there is an appropriate choice of $m=m(\alpha, M)$ in $\delta_{h}$ such that for $h>0$ sufficiently small, the mapping $T_{\alpha}^{h}$ is, in fact, a contraction map in the space $\mathcal{M}_{\alpha}^{h}$ under the infinity norm. The proposition will follow by the equivalence of the integral representation of (3.9).

We first prove that $T_{\alpha}^{h}$ is an operator (i.e. $T_{\alpha}^{h}: \mathcal{M}_{\alpha}^{h} \rightarrow \mathcal{M}_{\alpha}^{h}$ ) for an appropriate choice of $m=m(\alpha, M)$ and small enough $h$. For the estimates below, $C \equiv C(M)$. Since $\phi \in \mathcal{M}_{\alpha}^{h}$, we have

$$
\left|T_{\alpha}^{h}(\phi)\left(\tilde{x}, x_{3}\right)\right| \leq 2\left|x_{3}\right|, \quad \text { for each } \quad\left(\tilde{x}, x_{3}\right) \in \Omega_{h} .
$$

In addition, using a similar estimate to (3.5), we obtain

$$
\left|\operatorname{det} \nabla V_{\alpha}^{h}\left(\tilde{x}, T_{\alpha}^{h}\left(\tilde{x}, x_{3}\right)\right)-1\right| \leq C\left|T_{\alpha}^{h}\left(\tilde{x}, x_{3}\right)\right| \delta_{h}^{\min \{-\alpha, 0\}} \leq C\left|x_{3}\right| h^{\min \{-\alpha, 0\}} m^{\min \{-\alpha, 0\}}
$$

for $\alpha \in\{-1,0,1\}$. Thus, for $\alpha \in\{-1,0\}$, we need only enforce $m \geq 1$ and for $\alpha=1$ we enforce $m=m(\alpha, M) \geq \max \{2 C, 1\}$ to ensure $T_{\alpha}^{h}$ is an operator for small $h$.

It remains to prove that $T_{\alpha}^{h}$ is a contraction under the $L^{\infty}$ norm. Observe for $\phi, \psi \in \mathcal{M}_{\alpha}^{h}$,

$$
\begin{aligned}
\left|T_{\alpha}^{h}(\phi)\left(\tilde{x}, x_{3}\right)-T_{\alpha}^{h}(\psi)\left(\tilde{x}, x_{3}\right)\right| & \leq 4 \int_{0}^{\left|x_{3}\right|} \mid \operatorname{det}\left(\nabla V_{\alpha}^{h}(\tilde{x}, \psi(\tilde{x}, s))-\operatorname{det}\left(\nabla V_{\alpha}^{h}(\tilde{x}, \phi(\tilde{x}, s)) \mid d s\right.\right. \\
& \leq C \delta_{h}^{\min \{-\alpha, 0\}} \int_{0}^{\left|x_{3}\right|}|\psi(\tilde{x}, s)-\phi(\tilde{x}, s)| d s \\
& \leq C m^{\min \{-\alpha, 0\}} h^{\min \{-\alpha, 0\}} h\|\psi-\phi\|_{L^{\infty}\left(\Omega_{h}\right)}
\end{aligned}
$$

for any $\left(\tilde{x}, x_{3}\right) \in \Omega_{h}$. Here the first inequality uses the determinant constraint on $\mathcal{M}_{\alpha}^{h}$, the second uses the equation (3.4), and the third uses that $\delta_{h}=m h$. Finally, from this estimate, it is clear that we can choose $m=m(\alpha, M) \geq 1$ independent of $h$ (in fact $m=1$ suffices for $\alpha=-1,0$ as in the remark), such that for $h$ sufficiently small

$$
\left\|T_{\alpha}^{h}(\phi)-T_{\alpha}^{h}(\psi)\right\|_{L^{\infty}\left(\Omega_{h}\right)}<\|\psi-\phi\|_{L^{\infty}\left(\Omega_{h}\right)},
$$

i.e. it is a contraction map.

We now fix this $m=m(\alpha, M)$ and an $h>0$ sufficiently small. Since $T_{\alpha}^{h}$ is a contraction map, there exists a $\xi_{\alpha}^{h}$ such that

$$
\xi_{\alpha}^{h}=T_{\alpha}^{h}\left(\xi_{\alpha}^{h}\right)=\int_{0}^{x_{3}} \frac{1}{\operatorname{det}\left(\nabla V_{\alpha}^{h}\left(\tilde{x}, \xi_{\alpha}^{h}(\tilde{x}, s)\right)\right)} d s \quad \text { for each } \quad\left(\tilde{x}, x_{3}\right) \in \Omega_{h} .
$$

This is equivalent to the ordinary differential equation (3.9). The regularity $\xi_{\alpha}^{h} \in C^{1}\left(\bar{\Omega}_{h}, \mathbb{R}\right)$ follows from the regularity of $y_{\alpha}^{\delta_{h}}$ and $b_{\alpha}^{\delta_{h}}$. The estimates (3.10) follow from the fact that $\xi_{h}^{\alpha} \in \mathcal{M}_{\alpha}^{h}$. This completes the proof.

\subsection{Definition of three dimensional deformations for low energy constructions}

We now defined the three dimensional fields used in our subsequent energy arguments for actuation.

Definition of three dimensional deformation for nonisometric orgiami: Let $r>0$. We suppose $\omega \subset \mathbb{R}^{2}$ satisfies (1.9), $N_{0}^{h}$ satisfies (1.7) with the design $n_{0}$ as in (1.10) and (1.11), y $\in W^{1, \infty}\left(\omega, \mathbb{R}^{3}\right)$ is an origami deformation as in (1.12), and each $\tilde{F}_{\alpha}$ and $n_{0 \alpha}$ in these relations satisfies (1.13). In addition, we assume there exists a $y^{\delta} \in C^{3}\left(\bar{\omega}, \mathbb{R}^{3}\right)$ satisfying the approximations property (1.16). 
Hence, by Proposition 2.4, we obtain a vector field $b^{\delta} \in C^{2}\left(\bar{\omega}, \mathbb{R}^{3}\right)$ with the identities (2.27) and (2.28) and the estimate (2.29). Thus by Lemma 3.1 with $\alpha=1$, there exists a $m=m(\tilde{\nabla} y) \geq 1$ such that for $h>0$ sufficiently small there exists a $\xi^{h} \in C^{1}\left(\bar{\Omega}_{h}, \mathbb{R}\right)$ and an extension $Y^{h} \in C^{1}\left(\bar{\Omega}_{h}, \mathbb{R}^{3}\right)$ with the properties:

$$
\left\{\begin{array}{l}
Y^{h}:=y^{\delta_{h}}+\xi^{h} b^{\delta_{h}} \quad \text { with } \quad \delta_{h}=m h, \quad \operatorname{det} \nabla Y^{h}=1 \quad \text { on } \Omega_{h}, \\
\left|\xi^{h}-x_{3}\right| \leq C h^{-1}\left|x_{3}\right|^{2}, \quad\left|\partial_{3} \xi^{h}-1\right| \leq C h^{-1}\left|x_{3}\right|, \quad\left|\tilde{\nabla} \xi^{h}\right| \leq C h^{-2}\left|x_{3}\right|^{2} \quad \text { on } \Omega_{h}
\end{array}\right.
$$

for $C=C(\tilde{\nabla} y)>0$ independent of $h$. With this construction, we prove Theorem 1.4 in Section 3.3.

Definition of three dimensional deformation for lifted surfaces: Let $r>1$. We suppose $\left\{\varphi, y, n_{0}\right\}$ are as in the lifted surface ansatz (i.e. $y$ satsifying (1.20) and $n_{0}$ satisfying (1.22) for $\varphi$ as in (1.21) for some $m>0$ ) and $N_{0}^{h}$ is as in (1.7). For $\delta>0$ sufficiently small, there exists $\delta$-dependent functions $\left\{\varphi_{\delta}, y^{\delta}, n_{0}^{\delta}, b^{\delta}\right\}$ approximating this ansatz with the properties as detailed in Section 2.5. Hence, by Lemma 3.1 with $\alpha=0$, for $h>0$ sufficiently small, we set $\delta=h$ (Remark 3.3) and there exists a $\xi^{h} \in C^{1}\left(\bar{\Omega}_{h}, \mathbb{R}\right)$ and an extension $Y^{h} \in C^{1}\left(\bar{\Omega}_{h}, \mathbb{R}^{3}\right)$ with the properties:

$$
\left\{\begin{array}{l}
Y^{h}:=y^{h}+\xi^{h} b^{h}, \quad \operatorname{det} \nabla Y^{h}=1 \quad \text { on } \Omega_{h}, \\
\left|\xi^{h}-x_{3}\right| \leq C\left|x_{3}\right|^{2}, \quad\left|\partial_{3} \xi^{h}-1\right| \leq C\left|x_{3}\right|, \quad\left|\tilde{\nabla} \xi^{h}\right| \leq C h^{-1}\left|x_{3}\right|^{2} \quad \text { on } \Omega_{h}
\end{array}\right.
$$

for $C=C(\tilde{\nabla} y)>0$ independent of $h$. With this construction, we prove Theorem 1.8 in Section 3.4.

Definition of three dimensional deformation for smooth surfaces: Let $r>0$. We suppose that $n_{0} \in C^{2}\left(\bar{\omega}, \mathbb{S}^{2}\right)$ and $y \in C^{3}\left(\bar{\omega}, \mathbb{R}^{3}\right)$ such that $(\tilde{\nabla} y)^{T} \tilde{\nabla} y=\tilde{\ell}_{n_{0}}$ on $\omega$. Following Proposition A.5, there exists a $b \in C^{2}\left(\bar{\omega}, \mathbb{R}^{3}\right)$ such that $(\tilde{\nabla} y \mid b)^{T}(\tilde{\nabla} y \mid b)=\ell_{n_{0}}$ and $\operatorname{det}(\tilde{\nabla} y \mid b)=1$. The smoothness is due to the regularity of $n_{0}$ and $y$ by explicit differentiation of the parameterization in (A.5). Now $y$ and $b$ satisfy the hypotheses of Lemma 3.1 with $\alpha=-1$ since these fields are $h$-independent. Hence, for $h>0$ sufficiently small there exists a $\xi^{h} \in C^{1}\left(\bar{\Omega}_{h}, \mathbb{R}\right)$ and an extension $Y^{h} \in C^{1}\left(\bar{\Omega}_{h}, \mathbb{R}^{3}\right)$ with the properties:

$$
\left\{\begin{array}{l}
Y^{h}:=y+\xi^{h} b, \quad \operatorname{det} \nabla Y^{h}=1 \quad \text { on } \Omega_{h}, \\
\left|\xi^{h}-x_{3}\right| \leq C\left|x_{3}\right|^{2}, \quad\left|\partial_{3} \xi^{h}-1\right| \leq C\left|x_{3}\right|, \quad\left|\tilde{\nabla} \xi^{h}\right| \leq C\left|x_{3}\right|^{2} \quad \text { on } \Omega_{h}
\end{array}\right.
$$

for $C=C(\tilde{\nabla} y)>0$ independent of $h$. With this construction, we prove Corollary1.9 also in Section 3.4 .

\subsection{The $O\left(h^{2}\right)$ energy argument for nonisometric origami}

We turn now to the proof of Theorem 1.4. Here and in the following, we find it useful to introduce the notation

$$
W^{e}\left(F, n, n_{0}\right)=W_{n H}\left(\left(\ell_{n}^{f}\right)^{-1 / 2} F\left(\ell_{n_{0}}^{0}\right)^{1 / 2}\right)
$$

where

$$
W_{n H}(F):=\frac{\mu}{2} \begin{cases}|F|^{2}-3 & \text { if } \operatorname{det} F=1 \\ +\infty & \text { otherwise. }\end{cases}
$$

We consider any three dimensional deformation as in the definition for nonisometric origami above: 
Proof of Theorem 1.4. We first remark that $Y^{h}(\tilde{x}, 0)=y^{\delta_{h}}(\tilde{x})$ for every $\tilde{x} \in \omega$ since $\xi^{h}(\tilde{x}, 0)=0$ following the first estimate for $\xi^{h}$ in (3.11). Thus, it remains only to show that the energy scales as $O\left(h^{2}\right)$ for this deformation.

To this end, we first compute $\nabla Y^{h}$ explicitly. We find that

$$
\nabla Y^{h}=\left(\tilde{\nabla} y^{\delta_{h}} \mid 0\right)+\xi^{h}\left(\tilde{\nabla} b^{\delta_{h}} \mid 0\right)+\left(\partial_{1} \xi^{h} b^{\delta_{h}}\left|\partial_{2} \xi^{h} b^{\delta_{h}}\right| \partial_{3} \xi^{h} b^{\delta_{h}}\right),
$$

and note that from Proposition 2.4, $\tilde{\nabla} b^{\delta_{h}}=0$ on the set $\omega \backslash \tilde{\omega}_{\delta_{h}}$. It follows that $\xi^{h}=x_{3}$ on this set. Indeed, since $\operatorname{det} \nabla Y^{h}=1$, we find that on $\omega \backslash \tilde{\omega}_{\delta_{h}}$,

$$
1=\operatorname{det}\left(\left(\tilde{\nabla} y^{\delta_{h}} \mid 0\right)+\left(\partial_{1} \xi^{h} b^{\delta_{h}}\left|\partial_{2} \xi^{h} b^{\delta_{h}}\right| \partial_{3} \xi^{h} b^{\delta_{h}}\right)\right)=\partial_{3} \xi^{h} \operatorname{det}\left(\tilde{\nabla} y^{\delta_{h}} \mid b^{\delta_{h}}\right) .
$$

Also from Proposition 2.4, $\operatorname{det}\left(\tilde{\nabla} y^{\delta_{h}} \mid b^{\delta_{h}}\right)=1$. Thus, $\partial_{3} \xi^{h}=1$ on $\omega \backslash \tilde{\omega}_{\delta_{h}}$. Consequently, $\xi^{h}=x_{3}$ on this set since we have the condition $\xi^{h}(\tilde{x}, 0)=0$.

To recap, we find that

$$
\nabla Y^{h}=\left(\tilde{\nabla} y^{\delta_{h}} \mid b^{\delta_{h}}\right) \quad \text { on } \omega \backslash \tilde{\omega}_{\delta_{h}}
$$

On the exceptional set $\tilde{\omega}_{h}$, we find that

$$
\begin{aligned}
\left|\nabla Y^{h}\right| & =\left|\left(\tilde{\nabla} y^{\delta_{h}} \mid b^{\delta_{h}}\right)+\left(\partial_{3} \xi^{h}-1\right) b^{\delta_{h}} \otimes e_{3}+x_{3}\left(\nabla^{\prime} b^{\delta_{h}} \mid 0\right)+\left(\xi^{h}-x_{3}\right)\left(\tilde{\nabla} b^{\delta_{h}} \mid 0\right)+b^{\delta_{h}} \otimes \tilde{\nabla} \xi^{h}\right| \\
& \leq\left|\left(\tilde{\nabla} y^{\delta_{h}} \mid b^{\delta_{h}}\right)\right|+\left|\partial_{3} \xi^{h}-1\right|\left|b^{\delta_{h}}\right|+\left|x_{3}\right|\left|\tilde{\nabla} b^{\delta_{h}}\right|+\left|\xi^{h}-x_{3}\right|\left|\tilde{\nabla} b^{\delta_{h}}\right|+\left|b^{\delta_{h}}\right|\left|\tilde{\nabla} \xi^{h}\right| \\
& \leq C\left(1+h^{-1}\left|x_{3}\right|+h^{-2}\left|x_{3}\right|^{2}\right) \leq C
\end{aligned}
$$

where each $C=C(\tilde{\nabla} y, m(\tilde{\nabla} y))>0$ is independent of $h$. These estimates follow from the estimates (1.16), (2.29) in Proposition 2.4, and (3.11).

Now, we recall from Proposition 2.4 that $\left(\tilde{\nabla} y^{\delta_{h}} \mid b^{\delta_{h}}\right)^{T}\left(\tilde{\nabla} y^{\delta_{h}} \mid b^{\delta_{h}}\right)=\ell_{n_{0}}$ on $\omega \backslash \tilde{\omega}_{\delta_{h}}$. Thus,

$$
\begin{aligned}
W^{e}\left(\left(\tilde{\nabla} y^{\delta_{h}} \mid b^{\delta_{h}}\right), n^{h}, n_{0}\right) & \\
& =W_{n H}\left(\left(\ell_{n^{h}}^{f}\right)^{-1 / 2}\left(\tilde{\nabla} y^{h} \mid b^{h}\right)\left(\ell_{n_{0}}^{0}\right)^{1 / 2}\right)=0 \quad \text { on } \omega \backslash \tilde{\omega}_{\delta_{h}}
\end{aligned}
$$

following Proposition A.4 and the identity (3.13). Here, we have set

$$
n^{h}:=\frac{\left(\tilde{\nabla} y^{\delta_{h}} \mid b^{\delta_{h}}\right) n_{0}}{\left|\left(\tilde{\nabla} y^{\delta_{h}} \mid b^{\delta_{h}}\right) n_{0}\right|} \quad \text { on } \omega .
$$

Since the energy density (3.17) vanishes, we deduce from Proposition A.3 that

$$
\left(\ell_{n^{h}}^{f}\right)^{-1 / 2}\left(\tilde{\nabla} y^{h} \mid b^{h}\right)\left(\ell_{n_{0}}^{0}\right)^{1 / 2}=: R^{h} \in S O(3) \quad \text { on } \omega \backslash \tilde{\omega}_{\delta_{h}} .
$$

We have yet to account for the non-ideal terms on this set as $N_{0}^{h}$ in (1.7) is the appropriate argument for the energy density, not $n_{0}$. To do this, we exploit the observation in (3.18). Indeed, we set

$$
N^{h}:=\frac{\left(\nabla Y^{h}\right) N_{0}^{h}}{\left|\left(\nabla Y^{h}\right) N_{0}^{h}\right|} \text { on } \Omega_{h}
$$

and observe

$$
\left(\ell_{N_{0}^{h}}^{0}\right)^{1 / 2}=\left(\ell_{n_{0}}^{0}\right)^{1 / 2}+O\left(x_{3}\right)+O(h), \quad\left(\ell_{N^{h}}^{f}\right)^{-1 / 2}=\left(\ell_{n^{h}}^{f}\right)^{-1 / 2}+O\left(x_{3}\right)+O(h)
$$


following the scaling of the non-ideal terms in (1.7). Hence on $\omega \backslash \tilde{\omega}_{\delta_{h}}$, we find

$$
\begin{aligned}
W^{e}\left(\nabla Y^{h}, N^{h}, N_{0}^{h}\right) & =W_{n H}\left(\left(\ell_{N^{h}}^{f}\right)^{-1 / 2}\left(\tilde{\nabla} y^{\delta_{h}} \mid b^{\delta_{h}}\right)\left(\ell_{N_{0}^{h}}^{0}\right)^{1 / 2}\right) \\
& =W_{n H}\left(\left(\ell_{n^{h}}^{f}\right)^{-1 / 2}\left(\tilde{\nabla} y^{\delta_{h}} \mid b^{\delta_{h}}\right)\left(\ell_{n_{0}}^{0}\right)^{1 / 2}+O\left(x_{3}\right)+O(h)\right) \\
& =W_{n H}\left(\left(R^{h}\right)^{T}\left(\left(\ell_{n^{h}}^{f}\right)^{-1 / 2}\left(\tilde{\nabla} y^{\delta_{h}} \mid b^{\delta_{h}}\right)\left(\ell_{n_{0}}^{0}\right)^{1 / 2}+O\left(x_{3}\right)+O(h)\right)\right) \\
& =W_{n H}\left(I_{3 \times 3}+O\left(x_{3}\right)+O(h)\right) \leq O\left(h^{2}\right) .
\end{aligned}
$$

For the equalities, we used (3.15), (3.19), the frame invariance of $W_{n H}$, and (3.18). For the inequality, we used the estimate in Proposition A.2. Thus, on the set $\omega \backslash \tilde{\omega}_{\delta_{h}}$ the energy is at most bending for nonisometric origami.

Now, on the exceptional set $\tilde{\omega}_{\delta_{h}}$, we have

$$
W^{e}\left(\nabla Y^{h}, N^{h}, N_{0}^{h}\right) \leq c\left(\left|\nabla Y^{h}\right|^{2}+1\right) \leq C
$$

given the estimate in Proposition A.1 and (3.16). Thus, on the set $\tilde{\omega}_{h}$ characterizing the creases and junctions, the energy is stretching energy, but this set is small for nonisometric origami, i.e. $\left|\tilde{\omega}_{\delta_{h}}\right|=O\left(\delta_{h}\right)=O(h)$ from Proposition 2.4 since $\delta_{h}=m h$.

Combining the estimates (3.20) and (3.21), we conclude

$$
\begin{aligned}
\mathcal{I}_{N_{0}^{h}}^{h}\left(Y^{h}\right) & =\int_{-h / 2}^{h / 2} \int_{\tilde{\omega}_{\delta_{h}}} W^{e}\left(\nabla Y^{h}, N^{h}, N_{0}^{h}\right) d x+\int_{-h / 2}^{h / 2} \int_{\omega \backslash \tilde{\omega}_{\delta_{h}}} W^{e}\left(\nabla Y^{h}, N^{h}, N_{0}^{h}\right) d x \\
& \leq C h\left|\tilde{\omega}_{\delta_{h}}\right|+O\left(h^{3}\right) \leq O\left(h^{2}\right) .
\end{aligned}
$$

This completes the proof.

\subsection{The $O\left(h^{3}\right)$ energy argument for lifted surfaces and smooth surfaces}

We now prove Theorem 1.8 and Corollary 1.9. First for Theorem 1.8, we consider any three dimensional deformation as in the definition for lifted surfaces in Section 3.2.

Proof of Theorem 1.8. We first note the $Y^{h}(\tilde{x}, 0)=y^{h}(\tilde{x})$ for $\tilde{x} \in \omega$ since $\xi^{h}(\tilde{x}, 0)=0$ by the first estimate for $\xi^{h}$ in (3.12). Moreover, $\left\|y^{h}-y\right\|_{W^{1, \infty}} \leq O(h)$ was shown in Proposition 2.6. So it remains to prove only the $O\left(h^{3}\right)$ scaling of the energy $\mathcal{I}_{N_{0}^{h}}\left(Y^{h}\right)$.

We compute explicitly

$$
\begin{gathered}
\nabla Y^{h}=\left(\tilde{\nabla} y^{h} \mid b^{h}\right)+x_{3}\left(\tilde{\nabla} b^{h} \mid 0\right)+\left(\xi^{h}-x_{3}\right)\left(\tilde{\nabla} b^{h} \mid 0\right) \\
+\left(\partial_{3} \xi^{h}-1\right) b^{h} \otimes e_{3}+b^{h} \otimes \tilde{\nabla} \xi^{h}
\end{gathered}
$$

Hence, by the estimates on $\xi^{h}$ in (3.12) and the estimates on $b^{h}$ in Proposition 2.7, we conclude

$$
\nabla Y^{h}=\left(\tilde{\nabla} y^{h} \mid b^{h}\right)+O\left(x_{3}\right) .
$$

Now since Proposition 2.7 gives $\left(\tilde{\nabla} y^{h} \mid b^{h}\right)^{T}\left(\tilde{\nabla} y^{h} \mid b^{h}\right)=\ell_{n_{0}^{h}}$, we find by an identical argument as that which lead to (3.18) that

$$
\left(\ell_{n^{h}}^{f}\right)^{-1 / 2}\left(\tilde{\nabla} y^{h} \mid b^{h}\right)\left(\ell_{n_{0}^{h}}^{0}\right)^{1 / 2}=: R^{h} \in S O(3) \quad \text { on } \omega \quad \text { for } \quad n^{h}:=\frac{\left(\tilde{\nabla} y^{h} \mid b^{h}\right) n_{0}^{h}}{\left|\left(\tilde{\nabla} y^{h} \mid b^{h}\right) n_{0}^{h}\right|} \quad \text { on } \omega .
$$


Note $n^{h}$ here is defined with the approximation $n_{0}^{h}$ and not by the design field $n_{0}$ as in (3.18). Now, we let $N^{h}:=\left(\nabla Y^{h}\right) N_{0}^{h} /\left|\left(\nabla Y^{h}\right) N_{0}^{h}\right|$ on $\Omega_{h}$, and observe that

$$
\begin{aligned}
\left(\ell_{N_{0}^{h}}^{0}\right)^{1 / 2} & =\left(\ell_{n_{0}}^{0}\right)^{1 / 2}+O(h) \\
& =\left(\ell_{n_{0}^{h}}^{0}\right)^{1 / 2}+O(h),
\end{aligned}
$$

where the first equality follows from the non-ideal terms in (1.7) and the second follows since $\left\|n_{0}-n_{0}^{h}\right\|_{L^{\infty}} \leq O(h)$ by Proposition 2.6. Additionally given (3.22), we conclude

$$
\left(\ell_{N^{h}}^{f}\right)^{-1 / 2}=\left(\ell_{n^{h}}^{f}\right)^{-1 / 2}+O\left(x_{3}\right)+O(h)
$$

for $n^{h}$ as in (3.23). Hence, analogous to the reasoning of (3.20),

$$
\begin{aligned}
W^{e}\left(\nabla Y^{h}, N^{h}, N_{0}^{h}\right) & =W_{n H}\left(\left(\ell_{n^{h}}^{f}\right)^{-1 / 2}\left(\tilde{\nabla} y^{h} \mid b^{h}\right)\left(\ell_{n_{0}^{h}}^{0}\right)^{1 / 2}+O\left(x_{3}\right)+O(h)\right) \\
& =W_{n H}\left(\left(R^{h}\right)^{T}\left(\ell_{n^{h}}^{f}\right)^{-1 / 2}\left(\tilde{\nabla} y^{h} \mid b^{h}\right)\left(\ell_{n_{0}^{h}}^{0}\right)^{1 / 2}+O\left(x_{3}\right)+O(h)\right) \\
& =W_{n H}\left(I_{3 \times 3}+O\left(x_{3}\right)+O(h)\right) \leq O\left(h^{2}\right)
\end{aligned}
$$

for $R^{h}$ in (3.23). Since this inequality holds on all of $\omega$,

$$
\mathcal{I}_{N_{0}^{h}}^{h}\left(Y^{h}\right)=\int_{-h / 2}^{h / 2} \int_{\omega} W^{e}\left(\nabla Y^{h}, N^{h}, N_{0}^{h}\right) d x \leq O\left(h^{3}\right) .
$$

This completes the proof.

Now for Corollary 1.9, we consider any three dimensional deformation as in the definition for smooth surfaces in Section 3.2.

Proof of Corollary 1.9. We repeat the proof of Theorem 1.8 replacing $\left\{y^{h}, b^{h}, n_{0}^{h}\right\}$ with $\left\{y, b, n_{0}\right\}$ for these smooth surfaces and programs.

\section{Nonisometric origami and an opitimal scaling law}

In this section, we prove Theorem 1.6. Specifically, we show that for the two-dimensional analog to the entropic energy given by $\tilde{\mathcal{I}}_{n_{0}}^{h}$ in (1.18), a piecewise constant director design (with $\omega$ as in (1.9) and $n_{0}$ as in (1.10) and (1.11)) necessarily implies an energy of at least $O\left(h^{2}\right)$ upon actuation. In section 4.1, we show that this estimate can be reduced to a unit cell problem localized at a single interface. Further, we show that a lowerbound for this unit cell problem is described by a one-dimensional Modica-Mortola type functional. In their celebrated result, Modica and Mortola [35] (see also Modica [34]) prove that such functionals (under suitable hypothesis) $\Gamma$-converge to functionals which are proportional to the number of jumps of their argument. In our setting, these jumps correspond to the jump in the preferred metric over the interface. In Section 4.2, we present a self-contained argument which shows that the minimum of our Modica-Mortola type functional is necessarily bounded away from zero for $h>0$ sufficiently small. This is the key result we use to prove Theorem 1.6. 


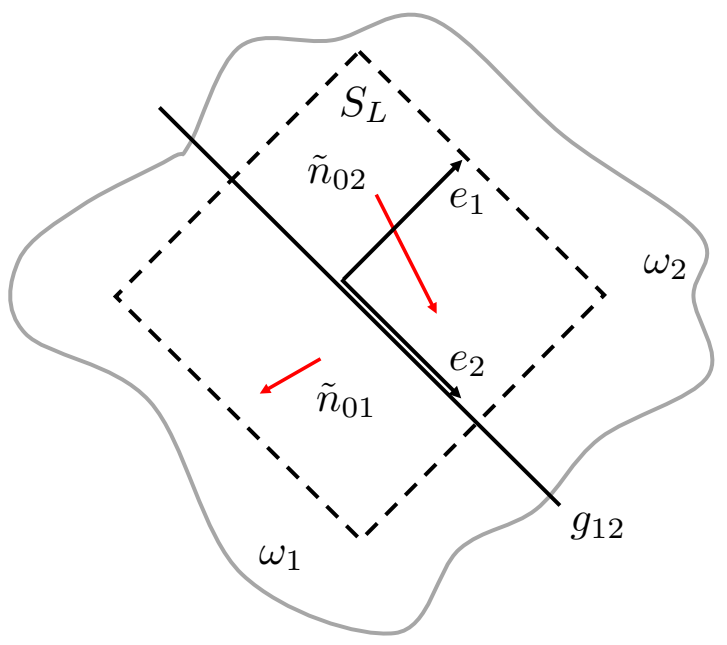

(a) Case 1

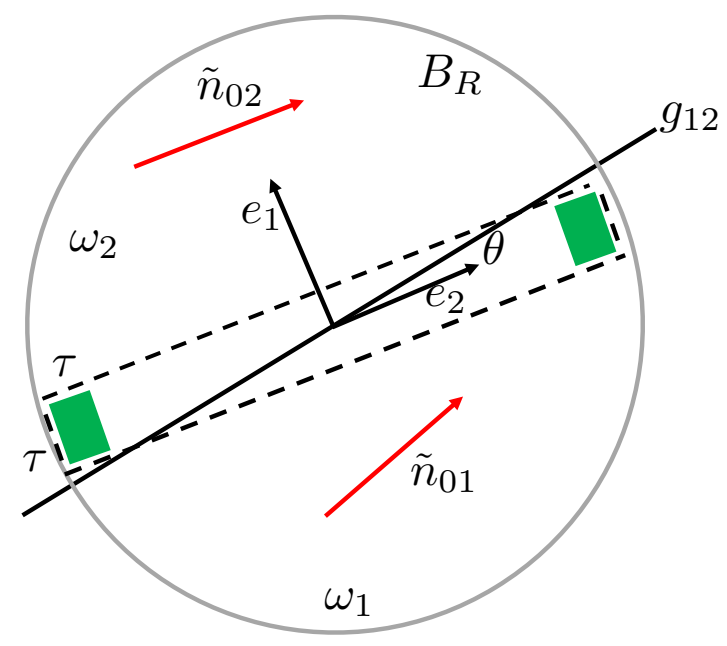

(b) Case 2

Figure 9: Schematic for unit cell problem of Theorem 1.6

\subsection{The unit cell problem}

We assume $\omega$ and $n_{0}: \omega \rightarrow \mathbb{S}^{2}$ satisfy (1.9), (1.10) and (1.11). Then there exists a straight interface $g_{\alpha \beta} \in \mathbb{S}^{1}$ adjoining two regions $\omega_{\alpha}$ and $\omega_{\beta}$ such that $\tilde{n}_{0 \alpha} \neq \pm \tilde{n}_{0 \beta}$. Focusing on this single interface, we have two cases to consider:

1. Case 1. $\left(\tilde{n}_{0 \alpha} \cdot e_{i}\right)^{2} \neq\left(\tilde{n}_{0 \beta} \cdot e_{i}\right)^{2}$ for at least one $i \in\{1,2\}$.

2. Case 2. $\left(\tilde{n}_{0 \alpha} \cdot e_{i}\right)^{2}=\left(\tilde{n}_{0 \beta} \cdot e_{i}\right)^{2}$ for both $i \in\{1,2\}$

Definition for Case 1: In this case, we relabel so that $\alpha=1$ and $\beta=2$. We fix a global frame so that $e_{2}$ lies on the $g_{12}$ interface and $e_{1}$ points in the direction of $\omega_{2}$. We let the origin of this frame lie on the $g_{12}$ interface such that for some $L>0$ there exists a $S_{L}:=(-L, L)^{2} \subset \omega_{1} \cup \omega_{2}$. A schematic of this description is provide in Figure 9a. We make the following observation in this case:

Proposition 4.1. If $\omega$ and $n_{0}$ have an interface as in the definition of Case 1 (see Figure 9a), then for any $y \in W^{2,2}\left(\omega, \mathbb{R}^{3}\right)$,

$$
\tilde{\mathcal{I}}_{n_{0}}^{h}(y) \geq 2 L^{2} h M_{1}^{h}
$$

where

$$
\begin{aligned}
M_{1}^{h}:= & \left\{\int_{-1}^{1}\left(\left(u^{2}-\sigma(t)\right)^{2}+\frac{h^{2}}{L^{2}}\left(u^{\prime}\right)^{2}\right) d t: u \in W^{1,2}((-1,1), \mathbb{R}) \text { with } u \geq 0 \text { a.e }\right\} \\
& \alpha(t)= \begin{cases}\alpha_{1} & \text { if } t<0 \\
\alpha_{2} & \text { if } t>0 .\end{cases}
\end{aligned}
$$

Here $\alpha_{1}, \alpha_{2} \geq 0$ and $\alpha_{1} \neq \alpha_{2}$.

Proof. Let $y \in W^{2,2}\left(\omega, \mathbb{R}^{3}\right)$. Since $S_{L} \subset \omega_{1} \cup \omega_{2} \subset \omega$ and the integrand in (1.18) is non-negative, we have

$$
\tilde{\mathcal{I}}_{n_{0}}^{h}(y) \geq h \int_{S_{L}}\left(\left|(\tilde{\nabla} y)^{T} \tilde{\nabla} y-\tilde{\ell}_{n_{0}}\right|^{2}+h^{2}|\tilde{\nabla} \tilde{\nabla} y|^{2}\right) d \tilde{x}
$$




$$
\begin{aligned}
& \geq h \int_{S_{L}}\left(\left|e_{i} \cdot\left((\tilde{\nabla} y)^{T} \tilde{\nabla} y-\tilde{\ell}_{n_{0}}\right) e_{i}\right|^{2}+h^{2}\left|\partial_{1} \partial_{i} y\right|^{2}\right) d \tilde{x} \\
& =h \int_{S_{L}}\left(\left.|| \partial_{i} y\right|^{2}+\left.\sigma\left(x_{1}\right)\right|^{2}+h^{2}\left|\partial_{1} \partial_{i} y\right|^{2}\right) d \tilde{x}
\end{aligned}
$$

where $i \in\{1,2\}$ is chosen such that $\left(\tilde{n}_{01} \cdot e_{i}\right)^{2} \neq\left(\tilde{n}_{02} \cdot e_{i}\right)^{2}$. We see then that $\sigma$ is given by

$$
\sigma(t)= \begin{cases}r^{-1 / 3}\left(1+(r-1)\left(\tilde{n}_{01} \cdot e_{i}\right)^{2}\right) & \text { if } t<0 \\ r^{-1 / 3}\left(1+(r-1)\left(\tilde{n}_{02} \cdot e_{i}\right)^{2}\right) & \text { if } t>0\end{cases}
$$

Thus, we set $\sigma_{1}:=r^{-1 / 3}\left(1+(r-1)\left(\tilde{n}_{01} \cdot e_{i}\right)^{2}\right)$ and $\sigma_{2}:=r^{-1 / 3}\left(1+(r-1)\left(\tilde{n}_{02} \cdot e_{i}\right)^{2}\right)$, and note that $\sigma_{1} \neq \sigma_{2}$ by definition of this case, and $\sigma_{1}, \sigma_{2}>0$ since $r>0$.

Given the chain of inequalities (4.1), we deduce that

$$
\begin{aligned}
\tilde{\mathcal{I}}_{n_{0}}^{h}(y) & \geq 2 \operatorname{Lh} \inf \left\{\int_{-L}^{L}\left(\left(|w|^{2}-\sigma(t)\right)^{2}+h^{2}\left|w^{\prime}\right|^{2}\right) d t: w \in W^{1,2}\left((-L, L), \mathbb{R}^{3}\right)\right\} \\
& \geq 2 L h \inf \left\{\int_{-L}^{L}\left(\left(|w|^{2}-\sigma(t)\right)^{2}+h^{2}\left(|w|^{\prime}\right)^{2}\right) d t: w \in W^{1,2}\left((-L, L), \mathbb{R}^{3}\right)\right\} \\
& =2 L h \inf \left\{\int_{-L}^{L}\left(\left(v^{2}-\sigma(t)\right)^{2}+h^{2}\left(v^{\prime}\right)^{2}\right) d t: v \in W^{1,2}((-L, L), \mathbb{R}) \quad \text { with } v \geq 0 \text { a.e. }\right\} \\
& =2 L^{2} h M_{1}^{h} .
\end{aligned}
$$

The first inequality follows by replacing $\partial_{2} y$ with a function $w$ which depends only on $x_{1}$ and taking the infimum amongst $W^{1,2}$ functions, and the second follows by noting $\left(|w|^{\prime}\right)^{2} \leq\left|w^{\prime}\right|^{2}$. Finally, we simply replace $|w|$ by a function $v \geq 0$ for the first equality, and the second equality follows by a change of variables $v(t)=u(t / L)$. This completes the proof.

Definition for Case 2: In this case, we again relabel so that $\alpha=1$ and $\beta=2$. We note that $\tilde{n}_{02} \neq 0$ (otherwise, following the definition of Case $2, \tilde{n}_{01}=0$ and therefore $\tilde{n}_{01}=\tilde{n}_{02}$ which is not allowed). Hence, we again fix a global Cartesian frame so that $e_{2}=\tilde{n}_{02} /\left|\tilde{n}_{02}\right|$ and $e_{1}$ points in the direction of region $\omega_{2}$. Next, for some $R>0$, we find a ball $B_{R} \subset \omega_{1} \cup \omega_{2}$ whose center intersects the interface $g_{12}$. Note that $R=R(\omega)$ depends only on $\omega$. We set $\theta \in(0, \pi / 2]$ to be the acute angle between $\tilde{n}_{02}$ and $g_{12}$ (which is non-zero by definition of this case) and define

$$
L_{1}:=R \cos (\theta), \quad \tau:=L_{1} \frac{\tan (\theta)}{1+\tan (\theta)} .
$$

We note that by their very definition, $L_{1}$ and $\tau$ depend only on $\omega$ and $n_{0}$. Further, $\tau \in\left(0, L_{1}\right]$. In particular, it cannot be zero since $\theta \neq 0$. A schematic of this case is provided in Figure $9 \mathrm{~b}$. We make the following observation for this case:

Proposition 4.2. If $\omega$ and $n_{0}$ have an interface as in the definition of Case 2 (see Figure 9b), then for any $y \in W^{2,2}\left(\omega, \mathbb{R}^{3}\right)$,

$$
\tilde{\mathcal{I}}_{n_{0}}^{h}(y) \geq L_{1} h \int_{-\tau}^{\tau} M_{2}^{h}(s) d s
$$

where

$$
M_{2}^{h}(s):=\left\{\int_{-1}^{1}\left(\left(u^{2}-\sigma(s, t)\right)^{2}+\frac{h^{2}}{L_{1}^{2}}\left(u^{\prime}\right)^{2}\right) d t: u \in W^{1,2}((-1,1), \mathbb{R}) \text { with } u \geq 0 \text { a.e. }\right\}
$$




$$
\sigma(s, t)= \begin{cases}\sigma_{1} & \text { if } t<\max \left\{-1+\tau / L_{1},\left(1-\tau / L_{1}\right)(s / \tau)\right\} \\ \sigma_{2} & \text { if } t>\min \left\{1-\tau / L_{1},\left(1-\tau / L_{1}\right)(s / \tau)\right\}\end{cases}
$$

Here $\sigma_{1}, \sigma_{2} \geq 0$ and $\sigma_{1} \neq \sigma_{2}$.

Proof. Let $y \in W^{2,2}\left(\omega, \mathbb{R}^{3}\right)$. Akin to the estimate in (4.1), we reason that

$$
\tilde{\mathcal{I}}_{n_{0}}^{h}(y) \geq 2 h \int_{-\tau}^{\tau} \int_{-L_{1}}^{L_{1}}\left(\left.|| \partial_{2} y\right|^{2}-\left.\sigma(\tilde{x})\right|^{2}+h^{2}\left|\partial_{2}^{2} y\right|^{2}\right) d \tilde{x}
$$

for $\sigma(\tilde{x})$ depending on both coordinates and given by

$$
\sigma(\tilde{x})= \begin{cases}r^{-1 / 3}\left(1+(r-1) \frac{\left(\tilde{n}_{01} \cdot \tilde{n}_{02}\right)^{2}}{\left|\tilde{n}_{02}\right|^{2}}\right) & \text { if } x_{2}<\max \left\{-L_{1}+\tau,\left(L_{1}-\tau\right)\left(x_{1} / \tau\right)\right\} \\ r^{-1 / 3}\left(1+(r-1)\left|\tilde{n}_{02}\right|^{2}\right) & \text { if } x_{2}>\min \left\{L_{1}-\tau,\left(L_{1}-\tau\right)\left(x_{1} / \tau\right)\right\} .\end{cases}
$$

Since $\tilde{n}_{01} \neq \pm \tilde{n}_{02}$ by definition, $\left(\tilde{n}_{01} \cdot \tilde{n}_{02}\right) \neq\left|\tilde{n}_{02}\right|^{2}$. Therefore, $\sigma_{1}:=r^{-1 / 3}\left(1+(r-1)\left|\tilde{n}_{02}\right|^{-2}\left(\tilde{n}_{01}\right.\right.$. $\left.\left.\tilde{n}_{02}\right)^{2}\right)$ does not equal $\sigma_{2}:=r^{-1 / 3}\left(1+(r-1)\left|\tilde{n}_{02}\right|^{2}\right)$. Moreover, $\sigma_{1}, \sigma_{2}>0$ since $r>0$.

Now, given the inequality in (4.2), we again see that in this case

$$
\begin{aligned}
\mathcal{I}_{n_{0}}^{h}(y) & \geq h \int_{-\tau}^{\tau} \inf \left\{\int_{-L_{1}}^{L_{1}}\left(\left(|w|^{2}-\sigma(s, t)\right)^{2}+h^{2}\left|w^{\prime}\right|^{2}\right) d t: w \in W^{1,2}\left(\left(-L_{1}, L_{1}\right), \mathbb{R}^{3}\right)\right\} d s \\
& \geq L_{1} h \int_{-\tau}^{\tau} M_{2}^{h}(s) d s
\end{aligned}
$$

as desired. This part of the argument is completely analogous to that of Proposition 4.1. This completes the proof.

\subsection{The Modica-Mortola analog and proof of optimal scaling}

We have shown that given any design described by flat sheet $\omega \subset \mathbb{R}^{2}$ and $n_{0}: \omega \rightarrow \mathbb{S}^{2}$ satisfying (1.9), (1.10) and (1.11), the problem of deducing a lowerbound on the energy (1.18) reduces to a unit cell problem which has at most two flavors: Case 1 and Case 2 in Section 4.1. Actually though, following Proposition 4.1 and 4.2, we find for the lowerbound that one only needs to consider the variational problem given by the one dimensional functionals

$$
\mathcal{I}_{s}^{h}(u):=\int_{-1}^{1} \frac{1}{h}\left(\left(u^{2}-\sigma(s, t)\right)^{2}+c_{1} h\left(u^{\prime}\right)^{2}\right) d t, \quad s \in\left[-c_{2}, c_{2}\right]
$$

minimized amongst the functions $\left\{u \in W^{1,2}((-1,1), \mathbb{R}): u \geq 0\right\}$ where

$$
\sigma(s, t)= \begin{cases}\sigma_{1} & \text { if } t<\max \left\{-1+c_{3}, c_{4} s\right\} \\ \sigma_{2} & \text { if } t>\min \left\{1-c_{3}, c_{4} s\right\}\end{cases}
$$

for $c_{1}, c_{2}>0, c_{3} \in(0,1]$ and $c_{4} \in\left[0,\left(1-c_{3}\right) / c_{2}\right)$. In fact, the proof of Theorem 1.6 follows from the observation that the infimum of $\mathcal{I}_{s}^{h}$ is bounded away from zero. Precisely:

Lemma 4.3. For any $c_{1}, c_{2}>0, c_{3} \in(0,1]$ and $c_{4} \in\left[0,\left(1-c_{3}\right) / c_{2}\right)$, and for $h>0$ sufficiently small

$$
\inf \left\{\mathcal{I}_{s}^{h}(u): u \in W^{1,2}((-1,1), \mathbb{R}) \text { with } u \geq 0 \text { a.e. }\right\} \geq c_{L}
$$

where $c_{L}=c_{L}\left(c_{1}, c_{2}, c_{3}, c_{4}\right)>0$ is independent of $s$ and $h$. 
This is the crucial observation for the theorem. Indeed:

Proof of Theorem 1.6. We note following Section 4.1 that it suffices to restrict to the unit cell problem given by the two cases in Figure 9. From Proposition 4.1 and 4.2, we have that for any $y \in W^{2,2}\left(\omega, \mathbb{R}^{3}\right)$,

$$
\mathcal{I}_{n_{0}}^{h}(y) \geq \begin{cases}2 L^{2} h M_{1}^{h} & \text { for Case } 1 \\ L_{1} h \int_{-\tau}^{\tau} M_{2}^{h}(s) d x & \text { for Case } 2 .\end{cases}
$$

In addition, we observe that

$$
\begin{gathered}
M_{1}^{h}=h \inf \left\{\mathcal{I}_{s}^{h}(u): u \in W^{1,2}((-1,1), \mathbb{R}) \text { with } u \geq 0 \text { a.e. }\right\} \\
\text { when } \quad c_{1}=L^{-1}, c_{3}=1, c_{4}=0 ; \\
M_{2}^{h}(s)=h \inf \left\{\mathcal{I}_{s}^{h}(u): u \in W^{1,2}((-1,1), \mathbb{R}) \text { with } u \geq 0 \text { a.e. }\right\} \\
\text { when } \quad c_{1}=L_{1}^{-1}, c_{2}=\tau, c_{3}=\tau / L_{1}, c_{4}=\left(1 / \tau-1 / L_{1}\right) .
\end{gathered}
$$

Thus, by these observations and given Lemma 4.3,

$$
\mathcal{I}_{n_{0}}^{h}(y) \geq \begin{cases}2 L^{2} c_{L} h^{2} & \text { for Case } 1 \\ 2 L_{1} \tau c_{L} h^{2} & \text { for Case } 2\end{cases}
$$

for $c_{L}=c_{L}\left(c_{1}, c_{2}, c_{3}, c_{4}\right)>0$ as in the lemma. This completes the proof.

To close the argument, it remains to prove Lemma 4.3. We do this now:

Proof of Lemma 4.3. By the direct methods in the calculus of variations (see, for instance, Dacorogna [19]), we find that for any $s \in\left[-c_{2}, c_{2}\right]$ and $h>0$, there exists a minimizer to $\mathcal{I}_{s}^{h}$ in the space $\left\{u \in W^{1,2}((-1,1), \mathbb{R}): u \geq 0\right.$ a.e. $\}$. For the lowerbound, it suffices to restrict our attention to any such minimizer, which we label as $u_{s}^{h}$. Further, we may assume for some fixed constant $M>0$ that

$$
\mathcal{I}_{s}^{h}\left(u_{s}^{h}\right)<M
$$

Indeed, if for some $s \in\left[-c_{2}, c_{2}\right]$ and $h>0$ this does not hold, then we immediately establish a lowerbound for this case since the reverse inequality holds.

Now, since $c_{4} \in\left[0,\left(1-c_{3}\right) / c_{2}\right)$, we have that $\sigma(s, t)=\sigma_{1}$ when $t<-1+c_{3}$ and $\sigma(s, t)=\sigma_{2}$ when $t>1-c_{3}$. Without loss of generality, we assume $\sigma_{1}<\sigma_{2}$. We let $\langle\sigma\rangle=\left(\sigma_{1}+\sigma_{2}\right) / 2$, and we claim that for $h>0$ sufficiently small,

$$
\left\{\begin{array}{lll}
\text { for some } & t \in\left[-1,-1+c_{3} / 2\right], & u_{s}^{h}(t)^{2} \in\left(\frac{1}{2} \sigma_{1}, \frac{1}{2}\left(\sigma_{1}+\langle\sigma\rangle\right)\right) ; \\
\text { for some } t \in\left[1-c_{3} / 2,1\right], & u_{s}^{h}(t)^{2} \in\left(\frac{1}{2}\left(\sigma_{2}+\langle\sigma\rangle\right), \frac{3}{2} \sigma_{2}\right) .
\end{array}\right.
$$

Indeed, suppose the first condition does not hold. Then $\left(u_{s}^{h}(t)^{2}-\sigma_{1}\right)^{2} \geq \frac{1}{4} \min \left\{\sigma_{1}^{2},\left(\langle\sigma\rangle-\sigma_{1}\right)^{2}\right\}>0$ on the interval $\left[-1,-1+c_{3} / 2\right]$ which gives

$$
\mathcal{I}_{s}^{h}\left(u_{s}^{h}\right) \geq \int_{-1}^{-1+c_{3} / 2} \frac{1}{h}\left(\left(u_{s}^{h}\right)^{2}-\sigma_{1}\right)^{2} d t \geq \frac{c_{3}}{8 h} \min \left\{\sigma_{1}^{2},\left(\langle\sigma\rangle-\sigma_{1}\right)^{2}\right\} .
$$


Taking $h>0$ sufficiently small, we eventually arrive at a contradiction to (4.3). The second condition in (4.4) holds by an identical argument.

Now, by the Sobolev embedding theorem $u_{s}^{h} \in W^{1,2}((-1,1), \mathbb{R})$ has a continuous representative. This continuity and the observation that (4.4) holds leads to the non-zero lowerbound on the energy. Indeed, we have the estimate

$$
\mathcal{I}_{s}^{h}\left(u_{s}^{h}\right) \geq 2 \sqrt{c_{1}} \int_{-1}^{1}\left|\left(u_{s}^{h}\right)^{2}-\sigma(s, t)\right|\left|\left(u_{s}^{h}\right)^{\prime}\right| d t .
$$

Hence, we define

$$
a:=\max \left\{t \in[-1,1]: u_{s}^{h}(t)^{2}=\frac{1}{2}\left(\sigma_{1}+\langle\sigma\rangle\right)\right\}, \quad b:=\min \left\{t \in(a, 1]: u_{s}^{h}(t)^{2}=\frac{1}{2}\left(\sigma_{2}+\langle\sigma\rangle\right)\right\} .
$$

7 By the continuity of $u_{s}^{h}$ and the observation (4.4), these quantities (as asserted) do, in fact, exist. Moreover,

$$
\begin{aligned}
\int_{-1}^{1}\left|\left(u_{s}^{h}\right)^{2}-\sigma(s, t)\right|\left|\left(u_{s}^{h}\right)^{\prime}\right| d t & \geq \int_{a}^{b}\left|\left(u_{s}^{h}\right)^{2}-\sigma(s, t)\right|\left|\left(u_{s}^{h}\right)^{\prime}\right| d t \geq \frac{1}{2} \min _{1,2}\left\{\left|\langle\sigma\rangle-\sigma_{i}\right|\right\} \int_{a}^{b}\left|\left(u_{s}^{h}\right)^{\prime}\right| d t \\
& \geq \frac{1}{2} \min _{1,2}\left\{\left|\langle\sigma\rangle-\sigma_{i}\right|\right\}\left|\int_{a}^{b}\left(u_{s}^{h}\right)^{\prime} d t\right|=\frac{1}{2} \min _{1,2}\left\{\left|\langle\sigma\rangle-\sigma_{i}\right|\right\}\left|u_{s}^{h}(b)-u_{s}^{h}(a)\right| \\
& =\frac{1}{2 \sqrt{2}} \min _{1,2}\left\{\left|\langle\sigma\rangle-\sigma_{i}\right|\right\}\left|\left(\sigma_{2}+\langle\sigma\rangle\right)^{1 / 2}-\left(\sigma_{1}-+\langle\sigma\rangle\right)^{1 / 2}\right|
\end{aligned}
$$

by the fundamental theorem of calculus. Since this lowerbound is positive and independent of $s$ and $h$, combining (4.5) and (4.6) completes the proof.

\section{Compactness for bending configurations and the metric constraint}

In this section, we prove that the metric constraint (1.6) is necessary for a configuration in pure bending when Frank elasticity is comparable to entropic elasticity at the bending scale, Theorem 1.12. In Section 5.1, we address some key preliminary results for this compactness, including a crucial lemma which is a consequence of Geometric Rigidity. In Section 5.2, we prove Theorem 1.12 .

\subsection{Preliminaries for compactness}

The key lemma which enables a proof of compactness in this setting is based on the seminal result of Geometric Rigidity by Friesecke, James and Müller [23], and generalization to non-Euclidean plates by Lewicka and Pakzad [30].

Lemma 5.1. Let $\omega \subset \mathbb{R}^{2}$ bounded and Lipschitz, and $r_{f}, r_{0}>1$ and $\tau \geq 0$. There exists a $C=C\left(\omega, r_{f}, r_{0}, \tau\right)>0$ with the following property: For every $h>0, \Omega_{h}:=\omega \times(-h / 2, h / 2)$, $Y^{h} \in W^{1,2}\left(\Omega_{h}, \mathbb{R}^{3}\right), N^{h} \in W^{1,2}\left(\Omega_{h}, \mathbb{S}^{2}\right)$ and $N_{0}^{h}$ as in $(1.7)$ with $n_{0} \in W^{1.2}\left(\omega, \mathbb{S}^{2}\right)$, there exists an associated matrix field $G^{h}: \omega \rightarrow \mathbb{R}^{3 \times 3}$ satisfying the estimates

$$
\begin{aligned}
& \frac{1}{h} \int_{\Omega_{h}}\left|G^{h}-\left(\ell_{N^{h}}^{f}\right)^{-1 / 2}\left(\nabla Y^{h}\right)\left(\ell_{N_{0}^{h}}^{0}\right)^{1 / 2}\right|^{2} d x \\
& \quad \leq \frac{C}{h} \int_{\Omega_{h}}\left(\operatorname{dist}^{2}\left(\left(\ell_{N^{h}}^{f}\right)^{-1 / 2}\left(\nabla Y^{h}\right)\left(\ell_{N_{0}^{h}}^{0}\right)^{1 / 2}, S O(3)\right)+h^{2}\left(\left|\nabla N^{h}\right|^{2}+\left|\tilde{\nabla} n_{0}\right|^{2}+1\right)\right) d x
\end{aligned}
$$




$$
\begin{aligned}
& \int_{\omega}\left|\tilde{\nabla} G^{h}\right|^{2} d \tilde{x} \\
& \quad \leq \frac{C}{h^{3}} \int_{\Omega_{h}}\left(\operatorname{dist}^{2}\left(\left(\ell_{N^{h}}^{f}\right)^{-1 / 2}\left(\nabla Y^{h}\right)\left(\ell_{N_{0}^{h}}^{0}\right)^{1 / 2}, S O(3)\right)+h^{2}\left(\left|\nabla N^{h}\right|^{2}+\left|\tilde{\nabla} n_{0}\right|^{2}+1\right)\right) d x .
\end{aligned}
$$

We address this result in Appendix E. For similar results related to non-Euclidean plates in a different context, see Lewicka et al. [10], [29] .

Now, in terms of the rescaled variables $W^{h}$ and $M_{0}^{h}$, we have:

Proposition 5.2. Let $\omega \subset \mathbb{R}^{2}$ bounded and Lipschitz, $r_{f}, r_{0}>1, \tau \geq 0$, and $\varepsilon_{h}$ as in (1.31). Let $W^{h} \in W^{1,2}\left(\Omega, \mathbb{R}^{3}\right), M^{h} \in W^{1,2}\left(\Omega, \mathbb{S}^{2}\right)$ and $M_{0}^{h}$ as in (1.32) for $n_{0} \in W^{1,2}\left(\omega, \mathbb{S}^{2}\right)$. There exists an associated matrix field $G^{h}: \omega \rightarrow \mathbb{R}^{3 \times 3}$ such that

$$
\begin{gathered}
\int_{\Omega}\left|G^{h}-\left(\ell_{M^{h}}^{f}\right)^{-1 / 2}\left(\nabla_{h} W^{h}\right)\left(\ell_{M_{0}^{h}}^{0}\right)^{1 / 2}\right|^{2} d z \leq C h^{2}\left(\mathcal{J}_{M_{0}^{h}}^{h, \varepsilon_{h}}\left(W^{h}\right)+\left\|\tilde{\nabla} n_{0}\right\|_{L^{2}(\omega)}^{2}+1\right) \\
\int_{\Omega}\left|\tilde{\nabla} G^{h}\right|^{2} d \tilde{z} \leq C\left(\mathcal{J}_{M_{0}^{h}}^{h, \varepsilon_{h}}\left(W^{h}\right)+\left\|\tilde{\nabla} n_{0}\right\|_{L^{2}(\omega)}^{2}+1\right)
\end{gathered}
$$

for $\delta_{h}, c_{l}$ as in (1.31) and some uniform $C=C\left(\omega, r_{f}, r_{0}, c_{l} \tau\right)$ which is independent of $h$.

Proof. Using Proposition A.3 and the identity (3.13), we find that

$$
\begin{aligned}
\int_{\Omega} \operatorname{dist}^{2}\left(\left(\ell_{M^{h}}^{f}\right)^{-1 / 2}\left(\nabla_{h} W^{h}\right)\left(\ell_{M_{0}^{h}}^{0}\right)^{1 / 2}, S O(3)\right) d z & \leq \int_{\Omega} \widehat{W}_{n H}\left(\left(\ell_{M^{h}}^{f}\right)^{-1 / 2}\left(\nabla_{h} W^{h}\right)\left(\ell_{M_{0}^{h}}^{0}\right)^{1 / 2}\right) d z \\
& \leq \int_{\Omega} \widehat{W}^{e}\left(\nabla_{h} W^{h}, M^{h}, M_{0}^{h}\right) d z
\end{aligned}
$$

where $\widehat{(\cdot)}=(2 / \mu)(\cdot)$. Since $\varepsilon_{h}$ as in $(1.31)$, we also find that

$$
\int_{\Omega} h^{2}\left|\nabla_{h} M^{h}\right|^{2} d z \leq \frac{1}{c_{l}^{2}} \int_{\Omega} \varepsilon_{h}^{2}\left|\nabla_{h} M^{h}\right|^{2} d z .
$$

Combining these two estimates, we find that

$$
\int_{\Omega}\left(\operatorname{dist}^{2}\left(\left(\ell_{M^{h}}^{f}\right)^{-1 / 2}\left(\nabla_{h} W^{h}\right)\left(\ell_{M_{0}^{h}}^{0}\right)^{1 / 2}, S O(3)\right)+h^{2}\left|\nabla_{h} M^{h}\right|^{2}\right) d z \leq C h^{2} \mathcal{J}_{M_{0}^{h}}^{h, \varepsilon_{h}}\left(W^{h}\right)
$$

for some uniform $C=C\left(c_{l}\right)$.

To obtain the desired estimates (5.1) and (5.2), we change variables via

$$
z(x):=\left(x_{1}, x_{2}, x_{3} / h\right), \quad x \in \Omega_{h},
$$

and (1.29), we apply Lemma 5.1, and the estimates follow from the bound (5.3).

\subsection{Compactness for comparable entropic and Frank elasticity in bending}

We turn now to the proof of Theorem 1.12. For clarity, we break up the proof into several steps.

Recall that for this theorem, we suppose $M_{0}^{h}$ as in $(1.32)$ with $n_{0} \in W^{1,2}\left(\omega, \mathbb{S}^{2}\right)$ and $\varepsilon_{h}$ as in (1.31). We consider a sequence $\left\{W^{h}\right\} \subset W^{1,2}\left(\Omega, \mathbb{R}^{3}\right)$ such that

$$
\mathcal{J}_{M_{0}^{h}}^{h, \varepsilon_{h}}\left(W^{h}\right) \leq C<\infty
$$

as $h \rightarrow 0$. The convergences stated in each step are for a suitably chosen subsequence as $h \rightarrow 0$. 
Step 1. $M_{0}^{h} \rightarrow n_{0}$ in $L^{2}\left(\Omega, \mathbb{S}^{2}\right)$, and $\left(\ell_{M_{0}^{h}}^{0}\right)^{ \pm 1 / 2} \rightarrow\left(\ell_{n_{0}}^{0}\right)^{ \pm 1 / 2}$ in $L^{2}\left(\Omega, \mathbb{R}^{3 \times 3}\right)$.

The first convergence is a trivial consequence of the definition of $M_{0}^{h}$ in (1.32). The second follows from the estimate $\left|\left(\ell_{\alpha}^{0}\right)^{ \pm 1 / 2}-\left(\ell_{\beta}^{0}\right)^{ \pm 1 / 2}\right| \leq C\left(r_{0}\right)|\alpha-\beta|$ for $\alpha, \beta \in \mathbb{S}^{2}$ and the first convergence.

Step 2. The functions

$$
M^{h}:=N\left(\nabla_{h} W^{h}, M_{0}^{h}\right) \quad \text { on } \Omega
$$

are in $W^{1,2}\left(\Omega, \mathbb{S}^{2}\right)$. Moreover, $M^{h} \rightarrow n$ in $W^{1,2}\left(\Omega, \mathbb{S}^{2}\right)$ for some $n$ independent of $z_{3}$ and $\left(\ell_{M^{h}}^{f}\right)^{ \pm 1 / 2} \rightarrow$ $\left(\ell_{n}^{f}\right)^{ \pm 1 / 2}$ in $L^{2}\left(\Omega, \mathbb{R}^{3 \times 3}\right)$.

$M^{h}$ is in $W^{1,2}\left(\Omega, \mathbb{S}^{2}\right)$ since the energy $\mathcal{J}_{M_{0}^{h}}^{h, \varepsilon_{h}}\left(W^{h}\right)$ is finite. In particular, for $h \ll 1$ we have

$$
\frac{1}{c_{l}^{2}} \int_{\Omega}\left|\nabla M^{h}\right|^{2} d z \leq \frac{1}{c_{l}^{2}} \int_{\Omega}\left(\left|\tilde{\nabla} M^{h}\right|^{2}+\frac{1}{h^{2}}\left|\partial_{3} M^{h}\right|^{2}\right) d z \leq \mathcal{J}_{M_{0}^{h}}^{h, \varepsilon_{h}}\left(W^{h}\right) \leq C
$$

for $C$ independent of $h$ by (5.5). Thus, up to a subsequence $M^{h} \rightarrow n$ in $W^{1,2}\left(\Omega, \mathbb{R}^{3}\right)$. By Rellich's theorem, taking a further subsequence (if necessary), we have strong convergence, $M^{h} \rightarrow n$ in $L^{2}\left(\Omega, \mathbb{R}^{3}\right)$. Since $M^{h} \in \mathbb{S}^{2}$ a.e., we deduce that $n \in \mathbb{S}^{2}$ a.e. by this strong convergence. Further, $n$ is independent of $z_{3}$ since by the estimate (5.6), we find $\partial_{3} M^{h} \rightarrow 0$ in $L^{2}\left(\Omega, \mathbb{R}^{3}\right)$, and therefore $\partial_{3} n=0$ a.e. by the uniqueness of the weak $W^{1,2}$ limit. The convergences of $\left(\ell_{M^{h}}^{f}\right)^{ \pm 1 / 2}$ follow by an argument similar to the convergences of $\left(\ell_{M_{0}^{h}}^{0}\right)^{ \pm 1 / 2}$ in Step 1.

Step 3. $\left(W^{h}-\frac{1}{|\Omega|} \int_{\Omega} W^{h} d z\right) \rightarrow y$ in $W^{1,2}\left(\Omega, \mathbb{R}^{3}\right)$ for some $y$ independent of $z_{3}$. Also, $h^{-1} \partial_{3} W^{h} \rightarrow b$ in $L^{2}\left(\Omega, \mathbb{R}^{3}\right)$.

We have

$$
\frac{1}{c} \int_{\Omega}\left(\left|\tilde{\nabla} W^{h}\right|^{2}+\left|h^{-1} \partial_{3} W^{h}\right|^{2}-1\right) d z \leq \int_{\Omega} W^{e}\left(\nabla_{h} W^{h}, M^{h}, M_{0}^{h}\right) d z \leq \mathcal{J}_{M_{0}^{h}}^{h, \varepsilon_{h}}\left(W^{h}\right) \leq C
$$

by Proposition A.1 and (5.5). Thus, since $\left|\nabla W^{h}\right| \leq\left|\nabla_{h} W^{h}\right|$ for $h \ll 1$, we conclude the first convergence (up to a subsequence) given the estimate (5.7) and an application of the Poincaré inequality. We again use (5.7) to conclude that up to a subsequence, $h^{-1} \partial_{3} W^{h} \rightarrow b$ in $L^{2}\left(\Omega, \mathbb{R}^{3}\right)$ for some vector valued function $b$, and that the limit $y$ is independent of $z_{3}$ (exactly the same argument as in Step 2 for $n$ independent of $z_{3}$ ).

Step 4. There exists a sequence of matrix fields $\left\{G^{h}\right\}$ with $G^{h}: \omega \rightarrow \mathbb{R}^{3 \times 3}$ such that

$$
\int_{\Omega}\left|G^{h}-\left(\ell_{M^{h}}^{f}\right)^{-1 / 2}\left(\nabla_{h} W^{h}\right)\left(\ell_{M_{0}^{h}}^{0}\right)^{1 / 2}\right|^{2} d z \leq C h^{2}, \quad \int_{\omega}\left|\tilde{\nabla} G^{h}\right|^{2} d \tilde{z} \leq C
$$

for $C$ independent of $h$. Moreover, $G^{h} \rightarrow R$ in $W^{1,2}\left(\omega, \mathbb{R}^{3 \times 3}\right)$ with $R \in S O(3)$ a.e.

To obtain the estimates in (5.8), we first apply Proposition 5.2 to obtain each matrix field $G^{h}$, and then observe that the estimates follow from the bound on the energy (5.5) and the fact that by hypothesis $n_{0} \in W^{1,2}\left(\omega, \mathbb{S}^{2}\right)$.

The first estimate in (5.8) implies

$$
\int_{\omega}\left|G^{h}\right|^{2} d \tilde{z} \leq C h^{2}+2 c\left(r_{f}, r_{0}\right) \int_{\Omega}\left|\nabla_{h} W^{h}\right|^{2} d z
$$

The constant $c\left(r_{f}, r_{0}\right)$ is from estimating the step-length tensors. From Step $3, \nabla_{h} W^{h}$ is bounded uniformly in $L^{2}$, and therefore using the above estimate and the second estimate in (5.8), we 
conclude that up to a subsequence $G^{h} \rightarrow R$ in $W^{1,2}\left(\omega, \mathbb{R}^{3 \times 3}\right)$. Now, to deduce that $R \in S O(3)$ a.e., we estimate via two applications of the triangle inequality

$$
\begin{aligned}
\int_{\omega} \operatorname{dist}^{2}(R, S O(3)) d \tilde{z} & \leq 2 \int_{\Omega}\left(\operatorname{dist}^{2}\left(G^{h}, S O(3)\right) d z+\left|G_{h}-R\right|^{2}\right) d z \\
& \leq C\left(h^{2}+\int_{\Omega}\left(\operatorname{dist}^{2}\left(\left(\ell_{M^{h}}^{f}\right)^{-1 / 2}\left(\nabla_{h} W^{h}\right)\left(\ell_{M_{0}^{h}}^{0}\right)^{1 / 2}, S O(3)\right)+\left|G_{h}-R\right|^{2}\right) d z\right) \\
& \leq C\left(h^{2}+h^{2} \mathcal{J}_{M_{0}^{h}}^{h, \varepsilon_{h}}\left(W^{h}\right)+\int_{\omega}\left|G_{h}-R\right|^{2} d \tilde{z}\right) .
\end{aligned}
$$

In the second estimate, we also use the first estimate in (5.8). For the third estimate, we recall (5.3). Now, by Rellich's theorem, we have $G^{h} \rightarrow R$ in $L^{2}\left(\Omega, \mathbb{R}^{3 \times 3}\right)$ for a subsequence. Thus, it is clear given (5.5) that the upperbound above vanishes as $h \rightarrow 0$. This implies $R \in S O(3)$ a.e. as desired.

Step 5. $\left(\ell_{M^{h}}^{f}\right)^{-1 / 2}\left(\nabla_{h} W^{h}\right)\left(\ell_{M_{0}^{h}}^{0}\right)^{1 / 2} \rightarrow R$ in $L^{2}\left(\Omega, \mathbb{R}^{3 \times 3}\right)$ for $R$ from Step 4 .

Since

$$
\begin{aligned}
& \int_{\Omega}\left|\left(\ell_{M^{h}}^{f}\right)^{-1 / 2}\left(\nabla_{h} W^{h}\right)\left(\ell_{M_{0}^{h}}^{0}\right)^{1 / 2}-R\right|^{2} d z \\
& \quad \leq 2 \int_{\Omega}\left(\left|G^{h}-R\right|^{2}+\left|G^{h}-\left(\ell_{M^{h}}^{f}\right)^{-1 / 2}\left(\nabla_{h} W^{h}\right)\left(\ell_{M_{0}^{h}}^{0}\right)^{1 / 2}\right|^{2}\right) d z
\end{aligned}
$$

we conclude that $\left(\ell_{M^{h}}^{f}\right)^{-1 / 2}\left(\nabla_{h} W^{h}\right)\left(\ell_{M_{0}^{h}}^{0}\right)^{1 / 2} \rightarrow R$ in $L^{2}\left(\Omega, \mathbb{R}^{3 \times 3}\right)$ using Step 4.

Step 6. Actually, $R=\left(\ell_{n}^{f}\right)^{-1 / 2}(\tilde{\nabla} y \mid b)\left(\ell_{n_{0}}^{0}\right)^{-1 / 2}$ a.e. for the limiting fields above. In particular, $\left(\ell_{n}^{f}\right)^{-1 / 2}(\tilde{\nabla} y \mid b)\left(\ell_{n_{0}}^{0}\right)^{1 / 2} \in W^{1,2}(\omega, S O(3))$.

We observe that $\left\|\left(\ell_{M^{h}}^{f}\right)^{-1 / 2}\left(\nabla_{h} W^{h}\right)\right\|_{L^{2}(\Omega)} \leq c\left(r_{f}\right)\left\|\nabla_{h} W^{h}\right\|_{L^{2}(\Omega)} \leq C$ by the compactness of the step-length tensor on $\mathbb{S}^{2}$ and following Step 3. So up to a subsequence $\left(\ell_{M^{h}}^{f}\right)^{-1 / 2}\left(\nabla_{h} W^{h}\right)$ converges weakly in $L^{2}\left(\Omega, \mathbb{R}^{3 \times 3}\right)$. In addition, the results of Step 2 and 3 imply $\left(\ell_{M^{h}}^{f}\right)^{-1 / 2}\left(\nabla_{h} W^{h}\right) \rightarrow$ $\left(\ell_{n}^{f}\right)^{-1 / 2}(\tilde{\nabla} y \mid b)$ in $L^{1}\left(\Omega, \mathbb{R}^{3 \times 3}\right)$. Hence, in combination and by the uniqueness of the $L^{1}$ limit, we also have weak convergence to this limiting field in $L^{2}$ (rather than just $L^{1}$ ).

Given the weak- $L^{2}$ convergence just established and the convergence in Step 1, we deduce

$$
\left(\ell_{M^{h}}^{f}\right)^{-1 / 2}\left(\nabla_{h} W^{h}\right)\left(\ell_{M_{0}^{h}}^{0}\right)^{1 / 2} \rightarrow\left(\ell_{n}^{f}\right)^{-1 / 2}(\tilde{\nabla} y \mid b)\left(\ell_{n_{0}}^{0}\right)^{1 / 2} \quad \text { in } L^{1}\left(\Omega, \mathbb{R}^{3 \times 3}\right) .
$$

By the convergence in Step 5 and the uniqueness of the weak- $L^{1}$ limit $R=\left(\ell_{n}^{f}\right)^{-1 / 2}(\tilde{\nabla} y \mid b)\left(\ell_{n_{0}}^{0}\right)^{1 / 2}$ a.e. To complete the proof, we recall from Step 4 that $R \in W^{1,2}\left(\omega, \mathbb{R}^{3 \times 3}\right)$ and that $R \in S O(3)$ a.e.

Step 7. The sequences in Step 3 actually converge strongly in their respective spaces. In addition, we have improved regularity: $y \in W^{2,2}\left(\omega, \mathbb{R}^{3}\right)$ and $b$ is independent of $z_{3}$ and in $W^{1,2}\left(\omega, \mathbb{R}^{3}\right)$.

For the strong $L^{2}$ convergence, we have the estimate

$$
\begin{aligned}
& \int_{\Omega}\left|\nabla_{h} W^{h}-(\tilde{\nabla} y \mid b)\right|^{2} d z \\
& \leq 2 \int_{\Omega}\left(\left|\nabla_{h} W^{h}-\left(\ell_{M^{h}}^{f}\right)^{1 / 2} R\left(\ell_{M_{0}^{h}}^{0}\right)^{-1 / 2}\right|^{2}+\left|\left(\ell_{M^{h}}^{f}\right)^{1 / 2} R\left(\ell_{M_{0}^{h}}^{0}\right)^{-1 / 2}-(\tilde{\nabla} y \mid b)\right|^{2}\right) d z
\end{aligned}
$$




$$
\leq C \int_{\Omega}\left(\left|\left(\ell_{M^{h}}^{f}\right)^{-1 / 2}\left(\nabla_{h} W^{h}\right)\left(\ell_{M_{0}^{h}}^{0}\right)^{-1 / 2}-R\right|^{2}+\left|\left(\ell_{M^{h}}^{f}\right)^{1 / 2}-\left(\ell_{n}^{f}\right)^{1 / 2}\right|^{2}+\left|\left(\ell_{M_{0}^{h}}^{0}\right)^{-1 / 2}-\left(\ell_{n_{0}}^{0}\right)^{-1 / 2}\right|^{2}\right) d z
$$

using that $R=\left(\ell_{n}^{f}\right)^{-1 / 2}(\tilde{\nabla} y \mid b)\left(\ell_{n_{0}}^{0}\right)^{1 / 2}$ a.e. from Step 6 , and that the step-length tensors are compact and invertible on $\mathbb{S}^{2}$. It's clear that the upper bound $\rightarrow 0$ as $h \rightarrow 0$ due to the strong- $L^{2}$ convergences of each term (established in the previous steps). Thus, $\nabla_{h} W^{h} \rightarrow(\tilde{\nabla} y \mid b)$ in $L^{2}\left(\Omega, \mathbb{R}^{3 \times 3}\right)$ as desired.

For the improved regularity, we see that

$$
(\tilde{\nabla} y \mid b)=\left(\ell_{n}^{f}\right)^{1 / 2} R\left(\ell_{n_{0}}^{0}\right)^{-1 / 2} \quad \text { a.e. on } \omega .
$$

Note that $R \in W^{1,2}$ from Step 4, $n \in W^{1,2}$ from Step 2, and $n_{0} \in W^{1,2}$ by assumption. By the structure of the step-length tensors, we also have that $\left(\ell_{n_{0}}^{0}\right)^{-1 / 2},\left(\ell_{n}^{f}\right)^{1 / 2} \in W^{1,2}$. Thus, the improved regularity is clear from differentiating the right side using the product rule for these Sobolev functions. Finally, $b$ is independent of $z_{3}$ since $\left(\ell_{n}^{f}\right)^{1 / 2} R\left(\ell_{n_{0}}^{0}\right)^{-1 / 2} e_{3}$ is independent of $z_{3}$.

Step 8. Actually,

$$
n=N\left((\tilde{\nabla} y \mid b), n_{0}\right)=\frac{(\tilde{\nabla} y \mid b) n_{0}}{\left|(\tilde{\nabla} y \mid b) n_{0}\right|} \quad \text { a.e. on } \omega \text {. }
$$

Since both $\nabla_{h} W^{h}$ and $M_{0}^{h}$ strongly converge in $L^{2}$, they converge (up to a subsequence) pointwise a.e. to the functions $(\tilde{\nabla} y \mid b)$ and $n_{0}$ respectively. $N\left(F, m_{0}\right)$ is continuous is on the set $\left\{\left(F, m_{0}\right) \in \mathbb{R}^{3 \times 3} \times \mathbb{S}^{2}: \operatorname{det} F=1\right\}$. By this continuity and the pointwise a.e. convergence, we conclude $N\left(\nabla_{h} W^{h}, M_{0}^{h}\right) \rightarrow N\left((\tilde{\nabla} y \mid b), n_{0}\right)$ in $L^{2}\left(\Omega, \mathbb{R}^{3 \times 3}\right)$ by Lebesgue dominated convergence. Considering the convergence to $n$ in Step 2, the equality follows.

Step 9. Finally,

$$
(\tilde{\nabla} y)^{T} \tilde{\nabla} y=\ell_{n_{0}} \text { a.e. on } \omega \text {. }
$$

From Step 6, $\left(\ell_{n}^{f}\right)^{-1 / 2}(\tilde{\nabla} y \mid b)\left(\ell_{n_{0}}^{0}\right)^{1 / 2} \in W^{1,2}(\omega, S O(3))$, and from Step 7, $n$ as in (5.9). Hence,

$$
\int_{\omega} W^{e}\left((\tilde{\nabla} y \mid b), n, n_{0}\right) d \tilde{z}=\int_{\omega} W_{n H}\left(\left(\ell_{n}^{f}\right)^{-1 / 2}(\tilde{\nabla} y \mid b)\left(\ell_{n_{0}}^{0}\right)^{1 / 2}\right) d \tilde{z}=0
$$

using the definitions of $W^{e}$ and $W_{n H}$, and since $W_{n H}$ vanishes on $S O(3)$. The conclusion (5.10) follows by Proposition A.4.

Proof of Theorem 1.12. The proof follows by the collection of steps above. In particular, Step 7 shows the strong convergence of $\left\{W^{h}\right\}$ and the desired regularity of the limiting field $y$ as a consequence of (5.5). Step 9 shows that the metric constraint must also be satisfied. This is the proof.

\section{Appendices}

\section{A Some facts about the entropic energy}

\section{A.1 Some estimates on the energy densities}

Proposition A.1. If $F \in \mathbb{R}^{3 \times 3}$ such that $\operatorname{det} F=1$ and $n_{0} \in \mathbb{S}^{2}$, then

$$
\frac{1}{c}\left(|F|^{2}-1\right) \leq W^{e}\left(F, n, n_{0}\right) \leq c\left(|F|^{2}+1\right)
$$


for some $c=c\left(r_{f}, r_{0}\right)>0$.

Proof. Since $\operatorname{det} F=1$ and $n_{0} \in \mathbb{S}^{2}$, we have $n=F n_{0} /\left|F n_{0}\right| \in \mathbb{S}^{2}$ and

$$
\begin{aligned}
W^{e}\left(F, n, n_{0}\right) & =W_{n H}\left(\left(\ell_{n}^{f}\right)^{-1 / 2} F\left(\ell_{n_{0}}^{0}\right)^{1 / 2}\right) \\
& \geq \frac{\mu}{2}\left(\frac{1}{3} \sigma_{\min }^{2}\left(\left(\ell_{n_{0}}^{0}\right)^{1 / 2}\right)\left|\left(\ell_{n}^{f}\right)^{-1 / 2} F\right|^{2}-3\right) \\
& \geq \frac{\mu}{2}\left(\frac{1}{9} \sigma_{\min }^{2}\left(\left(\ell_{n_{0}}^{0}\right)^{1 / 2}\right) \sigma_{\min }^{2}\left(\left(\ell_{n}^{f}\right)^{-1 / 2}\right)|F|^{2}-3\right) .
\end{aligned}
$$

We note that $\sigma_{\min }\left(\left(\ell_{n_{0}}^{0}\right)^{1 / 2}\right)$ is nonzero and depends only on $r_{0}$. Similarly, $\sigma_{\min }\left(\left(\ell_{n}^{f}\right)^{-1 / 2}\right)$ is nonzero and depends only on $r_{f}$. Thus, the lowerbound in (A.1) follows. The upperbound is similar.

Proposition A.2. Let $A \in \mathbb{R}^{3 \times 3}$ such that $\operatorname{det}\left(I_{3 \times 3}+A\right)=1$. We find

$$
W_{n H}\left(I_{3 \times 3}+A\right) \leq C\left(|A|^{2}+|A|^{3}\right),
$$

for $W_{n H}$ in (3.14) and for some uniform constant $C>0$.

Proof. For the inequality on $W_{n H}$, we note that since $\operatorname{det}\left(I_{3 \times 3}+A\right)=1$,

$$
\operatorname{Tr}(A)=-\operatorname{Tr}(\operatorname{cof} A)-\operatorname{det}(A)
$$

and $W_{n H}$ is finite. Hence,

$$
\begin{aligned}
W_{n H}(I+A) & =\frac{\mu}{2}\left(|I+A|^{2}-3\right) \\
& =\frac{\mu}{2}\left(|A|^{2}+2 \operatorname{Tr}(A)\right) \\
& =\frac{\mu}{2}\left(|A|^{2}-2 \operatorname{Tr}(\operatorname{cof} A)-2 \operatorname{det}(A)\right) .
\end{aligned}
$$

Since there exists a $C>0$ independent of $A$ such that $|\operatorname{Tr}(\operatorname{cof} A)| \leq C|A|^{2}$ and $|\operatorname{det}(A)| \leq C|A|^{3}$, we conclude (A.2) following the identity (A.3).

\section{A.2 Relating the metric and the step-length tensor}

Proposition A.3. The energy density $W_{n H}$ in (3.14) satisfies $W_{n H}(A) \geq \frac{\mu}{2} \operatorname{dist}^{2}(A, S O(3))$ for all $A \in \mathbb{R}^{3 \times 3}$.

Proof. We may assume $\operatorname{det} A=1$ as the bound holds trivially otherwise. Consequently and by the polar decomposition theorem, $A=R U$ for $R \in S O(3)$ and $U$ positive definite. Hence, we find $\operatorname{dist}(A, S O(3))=\left|U-I_{3 \times 3}\right|$. In addition, since $\operatorname{det} U=1$ we conclude

$$
\begin{aligned}
\frac{\mu}{2} \operatorname{dist}^{2}(A, S O(3)) & =\frac{\mu}{2}\left|U-I_{3 \times 3}\right|^{2} \\
& =\frac{\mu}{2}\left(|U|^{2}-2 \operatorname{Tr}(U)+3\right) \\
& \leq \frac{\mu}{2}\left(|U|^{2}-\inf \{\operatorname{Tr}(B): B \text { pos. def., det } B=1\}+3\right) \\
& =\frac{\mu}{2}\left(|U|^{2}-3\right)=W_{n H}(A) .
\end{aligned}
$$

Here, we used that the infimum above is attained at $B=I$. 
Proposition A.4. The energy density $W^{e}$ in (1.2) satisfies $W^{e}\left(F, F n_{0} /\left|F n_{0}\right|, n_{0}\right)=0$ if and only if $\operatorname{det} F=1$ and $F^{T} F=\ell_{n_{0}}$ for $\ell_{n_{0}}$ defined in (1.5). In addition, if these identities hold, then $F n_{0} /\left|F n_{0}\right|=R n_{0}$ where $R \in S O(3)$ is the unique rotation associated with the polar decomposition of $F$.

Proof. We first assume $W^{e}\left(F, F n_{0} /\left|F n_{0}\right|, n_{0}\right)=0$. Then $\operatorname{det} F=1, n_{0} \in \mathbb{S}^{2}$ and $\left|F n_{0}\right| \neq 0$. We set $n:=F n_{0} /\left|F n_{0}\right| \in \mathbb{S}^{2}$ and observe from (3.13),

$$
0=W^{e}\left(F, F n_{0} /\left|F n_{0}\right|, n_{0}\right)=W_{n H}\left(\left(\ell_{n}^{f}\right)^{-1 / 2} F\left(\ell_{n_{0}}^{0}\right)^{1 / 2}\right) .
$$

Thus, we deduce from Proposition A.3 that $\left(\ell_{n}^{f}\right)^{-1 / 2} F\left(\ell_{n_{0}}^{0}\right)^{1 / 2}=R$ for some $R \in S O(3)$. Evidently then,

$$
F=\left(\ell_{n}^{f}\right)^{1 / 2} R\left(\ell_{n_{0}}^{0}\right)^{-1 / 2}
$$

Further,

$$
\begin{aligned}
r_{f}^{1 / 6} n=\left(\ell_{n}^{f}\right)^{-1 / 2} n & =\left(\ell_{n}^{f}\right)^{-1 / 2}\left(\frac{F n_{0}}{\left|F n_{0}\right|}\right) \\
& =\left(\ell_{n}^{f}\right)^{-1 / 2}\left(\frac{\left(\ell_{n}^{f}\right)^{1 / 2} R\left(\ell_{n_{0}}^{0}\right)^{-1 / 2} n_{0}}{\left|F n_{0}\right|}\right)=r_{0}^{-1 / 6}\left(\frac{R n_{0}}{\left|F n_{0}\right|}\right) .
\end{aligned}
$$

Here, we used the definition of $n$, the result in (A.4) and properties of the step-length tensors (1.3). Since both $n$ and $R n_{0} \in \mathbb{S}^{2}$, it follows from this equality chain that actually $n=R n_{0}$. Substituting this into (A.4) yields

$$
F=R\left(\ell_{n_{0}}^{f}\right)^{1 / 2} R^{T} R\left(\ell_{n_{0}}^{0}\right)^{-1 / 2}=R\left(\ell_{n_{0}}^{f}\right)^{1 / 2}\left(\ell_{n_{0}}^{0}\right)^{-1 / 2}=R \ell_{n_{0}}^{1 / 2}
$$

noting that $\left(\ell_{R n_{0}}^{f}\right)^{1 / 2}=R\left(\ell_{n_{0}}^{f}\right)^{1 / 2} R^{T}$ and $\left(\ell_{n_{0}}^{f}\right)^{1 / 2}\left(\ell_{n_{0}}^{0}\right)^{-1 / 2}=\ell_{n_{0}}^{1 / 2}$. Consequently, $F^{T} F=\ell_{n_{0}}$ as desired.

For the other direction, we assume $\operatorname{det} F=1$ and $F^{T} F=\ell_{n_{0}}$. This implies $F=R \ell_{n_{0}}^{1 / 2}$ for some $R \in S O(3)$ and $n:=F n_{0} /\left|F n_{0}\right| \in \mathbb{S}^{2}$. Thus,

$$
n=\frac{F n_{0}}{\left|F n_{0}\right|}=\frac{R \ell_{n_{0}}^{1 / 2} n_{0}}{\left|F n_{0}\right|}=\bar{r}^{-1 / 6} \frac{R n_{0}}{\left|F n_{0}\right|},
$$

and since both $n$ and $R n_{0} \in \mathbb{S}^{2}$, we deduce $n=R n_{0}$. Then by definition (1.2), $W^{e}\left(F, F n_{0} /\left|F n_{0}\right|, n_{0}=\right.$ $W^{e}\left(F, R n_{0}, n_{0}\right)$ and clearly this is finite. Further given (3.13), we find

$$
\begin{aligned}
W^{e}\left(F, R n_{0}, n_{0}\right) & =W_{n H}\left(\left(\ell_{R n_{0}}^{f}\right)^{-1 / 2} F\left(\ell_{n_{0}}^{0}\right)^{1 / 2}\right) \\
& =W_{n H}\left(R\left(\ell_{n_{0}}^{f}\right)^{-1 / 2} R^{T} R \ell_{n_{0}}^{1 / 2}\left(\ell_{n_{0}}^{0}\right)^{1 / 2}\right) \\
& =W_{n H}\left(R\left(\ell_{n_{0}}^{f}\right)^{-1 / 2}\left(\ell_{n_{0}}^{f}\right)^{1 / 2}\left(\ell_{n_{0}}^{0}\right)^{-1 / 2}\left(\ell_{n_{0}}^{0}\right)^{1 / 2}\right)=W_{n H}(R)
\end{aligned}
$$

For this, we have exploited properties of the step-length tensors (see previous paragraph). Hence since $R \in S O(3)$, by Proposition A.3 we find $W_{n H}(R)=0$ as desired.

Finally for the implication, we note that in the proof of both directions, we found $F=R \ell_{n_{0}}^{1 / 2}$ and $n=R n_{0}$ for $R \in S O(3)$. Consequently, since $\ell_{n_{0}}^{1 / 2}$ is positive definite, $R$ is actually the unique rotation in the polar decomposition of $F$. 
Proposition A.5. If $\tilde{F} \in \mathbb{R}^{3 \times 2}$ and $n_{0} \in \mathbb{S}^{2}$ such that $\tilde{F}^{T} \tilde{F}=\tilde{\ell}_{n_{0}}$, then there exists a $b \in \mathbb{R}^{3}$ such that

$$
(\tilde{F} \mid b)^{T}(\tilde{F} \mid b)=\ell_{n_{0}}, \quad \operatorname{det}(\tilde{F} \mid b)=1 .
$$

In particular,

$$
\begin{aligned}
& b=\bar{b}_{1} \tilde{F} e_{1}+\bar{b}_{2} \tilde{F} e_{2}+\bar{b}_{3}\left(\tilde{F} e_{1} \times \tilde{F} e_{2}\right), \\
& \left(\begin{array}{c}
\bar{b}_{1} \\
\bar{b}_{2}
\end{array}\right)=\left(\tilde{\ell}_{n_{0}}\right)^{-1} I_{2 \times 3} \ell_{n_{0}} e_{3}, \quad \bar{b}_{3}=\operatorname{det}\left(\left(\tilde{\ell}_{n_{0}}\right)^{-1}\right), \\
& \left(\tilde{\ell}_{n_{0}}\right)^{-1}=\bar{r}^{1 / 3}\left(I_{2 \times 2}+\left(\frac{1-r}{1+\left|\tilde{n}_{0}\right|^{2}(r-1)}\right) \tilde{n}_{0} \otimes \tilde{n}_{0}\right) .
\end{aligned}
$$

for $\tilde{n}_{0}=\left(n_{0} \cdot e_{1}, n_{0} \cdot e_{2}\right) \in B_{1}(0) \subset \mathbb{R}^{2}$.

Proof. We remark that $\operatorname{det}\left(\tilde{\ell}_{n_{0}}\right)=r^{-2 / 3}\left(1+(r-1)\left|\hat{n}_{0}\right|^{2}\right)>0$ for $r>0$. Thus $\operatorname{rank} \tilde{F}=2$ since by hypothesis $\tilde{F}^{T} \tilde{F}=\tilde{\ell}_{n_{0}}$. Therefore, $\operatorname{span}\left\{\tilde{F} e_{1}, \tilde{F} e_{2}, \tilde{F} e_{1} \times \tilde{F} e_{2}\right\}=\mathbb{R}^{3}$. Hence, (A.5) simply rewrites $b \in \mathbb{R}^{3}$ equivalently in terms of $\left(\bar{b}_{1}, \bar{b}_{2}, \bar{b}_{3}\right) \in \mathbb{R}^{3}$. The proof follows by explicitly verifying the formulae.

\section{B Proof of Proposition 1.10}

Here we prove Proposition 1.10 which gives an equivalent rewriting of the metric constraint in a form that is more useful for design. In fact we prove slightly more, as detailed in the following

Proposition B.1. Let $r \in(0,1)$ or $>1$. $\tilde{F} \in \mathbb{R}^{3 \times 2}$ and $m \in \mathbb{S}^{2}$ satisfy $\tilde{F}^{T} \tilde{F}=\tilde{\ell}_{m}$ if and only if

$$
\tilde{F} \in \mathcal{D}_{r}, \quad m \in \mathcal{N}_{\tilde{F}}^{r}
$$

for $\mathcal{D}_{r}$ defined in (1.24) and $\mathcal{N}_{\tilde{F}}^{r}$ defined in (1.25). Further, if $\tilde{F} \in \mathcal{D}_{r}$, then there exists an $m \in \mathcal{N}_{\tilde{F}}^{r}$.

Proof. Let $\tilde{F} \in \mathbb{R}^{3 \times 2}$ and $m \in \mathbb{S}^{2}$ satisfy $\tilde{F}^{T} \tilde{F}=\tilde{\ell}_{m}$. Equivalently,

$$
\left(\begin{array}{cc}
\left|\tilde{F} \tilde{e}_{1}\right|^{2} & \left(\tilde{F} \tilde{e}_{1} \cdot \tilde{F} \tilde{e}_{2}\right) \\
\left(\tilde{F} \tilde{e}_{1} \cdot \tilde{F} \tilde{e}_{2}\right) & \left|\tilde{F} \tilde{e}_{2}\right|^{2}
\end{array}\right)=r^{-1 / 3}\left(\begin{array}{cc}
1+(r-1)\left(m \cdot e_{1}\right)^{2} & (r-1)\left(m \cdot e_{1}\right)\left(m \cdot e_{2}\right) \\
(r-1)\left(m \cdot e_{1}\right)\left(m \cdot e_{2}\right) & 1+(r-1)\left(m \cdot e_{2}\right)^{2}
\end{array}\right)
$$

for $\left\{\tilde{e}_{1}, \tilde{e}_{2}\right\}$ and $\left\{e_{1}, e_{2}, e_{3}\right\}$ the standard basis on $\mathbb{R}^{2}$ and $\mathbb{R}^{3}$ respectively. Now, since $m \in \mathbb{S}^{2}$, $\left(m \cdot e_{\alpha}\right)^{2} \in[0,1]$ and

$$
\begin{aligned}
\left|\tilde{F} \tilde{e}_{\alpha}\right|^{2} & \in\left[r^{-1 / 3}, r^{2 / 3}\right] \quad \text { if } \quad r>1, \\
& \in\left[r^{2 / 3}, r^{-1 / 3}\right] \quad \text { if } \quad r<1,
\end{aligned}
$$

from (B.1) for $\alpha=1,2$. In addition, $\left(m \cdot e_{1}\right)^{2}+\left(m \cdot e_{2}\right)^{2} \leq 1$, and so

$$
\begin{aligned}
|\tilde{F}|^{2}=\left|\tilde{F} \tilde{e}_{1}\right|^{2}+\left|\tilde{F} \tilde{e}_{2}\right|^{2} & \leq r^{2 / 3}+r^{-1 / 3} \quad \text { if } \quad \bar{r}>1 \\
& \geq r^{2 / 3}+r^{-1 / 3} \quad \text { if } \quad r<1
\end{aligned}
$$

also from (B.1). Now note that substituting the diagonal terms into the square of the off diagonal term in (B.1) results in

$$
\left(\tilde{F} \tilde{e}_{1} \cdot \tilde{F} \tilde{e}_{2}\right)^{2}=\left(\left|\tilde{F} \tilde{e}_{1}\right|^{2}-r^{-1 / 3}\right)\left(\left|\tilde{F} \tilde{e}_{2}\right|^{2}-r^{-1 / 3}\right) .
$$




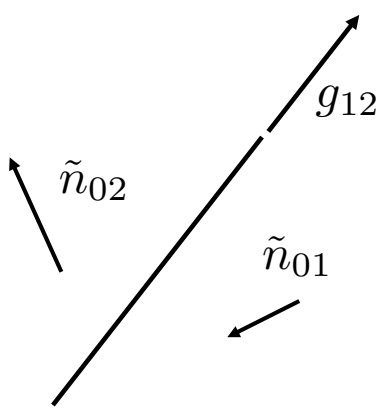

(a) A single interface

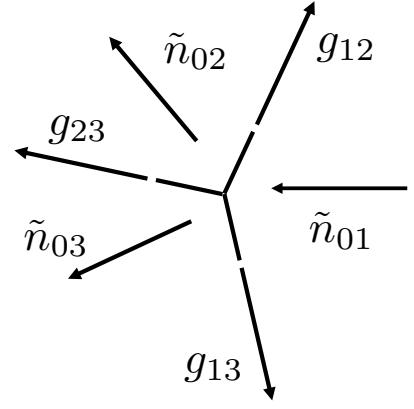

(b) Three-faced junction

Figure 10: Schematic of interfaces and junctions in nonisometric origami

Combining (B.2), (B.3) and (B.4), we conclude $\tilde{F} \in \mathcal{D}_{r}$ as desired. To prove $m \in \mathcal{N}_{\tilde{F}}^{r}$, note that since $r \neq 1$, rearranging the diagonal terms in (B.1) gives

$$
\left(m \cdot e_{\alpha}\right)^{2}=\frac{\left|\tilde{F} \tilde{e}_{\alpha}\right|^{2}-r^{-1 / 3}}{r^{2 / 3}-r^{-1 / 3}}, \quad \alpha=1,2 .
$$

Further, since $r>0$ and $\neq 1$, taking the sign of the off diagonal term in (B.1) gives

$$
\operatorname{sgn}\left(\left(m \cdot e_{1}\right)\left(m \cdot e_{2}\right)\right)=\operatorname{sgn}(r-1) \operatorname{sgn}\left(\tilde{F} \tilde{e}_{1} \cdot \tilde{F} \tilde{e}_{2}\right) .
$$

Since $m \in \mathbb{S}^{2}$, combining (B.5) and (B.6) yields $m \in \mathcal{N}_{\tilde{F}}^{r}$.

Now, let $\tilde{F} \in \mathcal{D}_{r}$ and $m \in \mathcal{N}_{\tilde{F}}^{r}$. To prove $\tilde{F}^{T} \tilde{F}=\tilde{\ell}_{m}$, we need to show (B.1). By hypothesis, we have (B.5). By rearranging this formula, we obtain the diagonal terms in (B.1). For the off diagonal term, we note that in addition to (B.5), we have (B.4) by hypothesis. Combining these relations, we find

$$
\left(\tilde{F} \tilde{e}_{1} \cdot \tilde{F} \tilde{e}_{2}\right)^{2}=\left(r^{2 / 3}-r^{-1 / 3}\right)^{2}\left(m \cdot e_{1}\right)^{2}\left(m \cdot e_{2}\right)^{2} .
$$

Taking the square root, we have the off diagonal term up to the sign. The correct choice of sign is guaranteed since $m$ and $\tilde{F}$ satisfy (B.6), again by hypothesis.

Finally, we let $\tilde{F} \in \mathcal{D}_{r}$, and show $\mathcal{N}_{\tilde{F}}^{r}$ is non-empty. Indeed by definition, $\tilde{F}$ satisfies (B.2) and (B.3). Thus, the right side of (B.5) is non-negative. From this, we may find an $m \in \mathbb{R}^{3}$ satisfying (B.5) and (B.6). Further by (B.3), $\left(m \cdot e_{1}\right)^{2}+\left(m \cdot e_{2}\right)^{2} \leq 1$. Thus, we can choose $\left(m \cdot e_{3}\right)$ such that $m \in \mathbb{S}^{2}$. It follows that $\mathcal{N}_{\tilde{F}}^{r}$ is non-empty.

\section{On nonisometric origami}

The actuation of complex origami shape stems from satisfying the nonisometric condition (1.13), hence the term nonisometric origami. In particular, the compatibility of interfaces separating regions of distinct constant director (Figure 10a) combined with the compatibility of junctions where these interfaces merge at a single point (Figure 10b) play the key role in actuation. To address this with mathematical precision, we note that (1.13) is equivalent to

$$
\tilde{F}_{\alpha}=R_{\alpha}\left(\ell_{n_{0 \alpha}}^{1 / 2}\right)_{3 \times 2} \quad \text { for some } R_{\alpha} \in S O(3), \quad \alpha \in\{1, \ldots, N\}
$$


where $\left(\ell_{n_{0 \alpha}}^{1 / 2}\right)_{3 \times 2}=r^{-1 / 6}\left(I_{3 \times 2}+\left(r^{1 / 2}-1\right) n_{0 \alpha} \otimes \tilde{n}_{0 \alpha}\right)$ for the projection $\tilde{n}_{0 \alpha} \in B_{1}(0) \subset \mathbb{R}^{2}$. Thus for compatibility, the deformation $y$ in (1.12) must be continuous across each interface separating regions of distinct constant director. This occurs if and only if

$$
R_{\alpha}\left(\ell_{n_{0 \alpha}}^{1 / 2}\right)_{3 \times 2} \tilde{g}_{\alpha \beta}=R_{\beta}\left(\ell_{n_{0 \beta}}^{1 / 2}\right)_{3 \times 2} \tilde{g}_{\alpha \beta}
$$

for every interface tangent $\tilde{g}_{\alpha \beta} \in \mathbb{S}^{1}$. Explicitly, $\tilde{g}_{\alpha \beta}$ represents the tangent vector to the interface separating regions $\omega_{\alpha}$ with director $n_{0 \alpha}$ and $\omega_{\beta}$ with director $n_{0 \beta}$ as depicted in Figure 10. This condition is akin to the rank-one condition studied in the context of fine-scale twinning during the austenite martensite phase transition and actuation active martensitic sheets [2],[8],[9]. More recently, this compatibility has been appreciated as a means of actuation for nematic elastomer and glass sheets [32],[33] using planar programming of the director. Here though, (C.1) describes the most general case of compatibility in thin nematic sheets as $n_{0 \alpha}, n_{0 \beta} \in \mathbb{S}^{2}$ need not be planar.

While (C.1) encodes a complete description of nonisometric origami as defined by (1.12) and (1.13), more useful criterion are gleamed from examining necessary and sufficient conditions associated with this constraint. In particular, taking the norm of both sides of (C.1) yields, after some manipulation, a necessary condition for nonisometric origami,

$$
\left|\tilde{n}_{0 \alpha} \cdot \tilde{g}_{\alpha \beta}\right|=\left|\tilde{n}_{0 \beta} \cdot \tilde{g}_{\alpha \beta}\right|
$$

for every interface tangent $\tilde{g}_{\alpha \beta}$ (when $r \neq 1$ ). We emphasize again that $\tilde{n}_{0 \alpha} \in B_{1}(0) \subset \mathbb{R}^{2}$ is the projection of $n_{0 \alpha}$ onto the tangent plane of $\omega$. That this need not be a unit vector is a direct consequence of allowing for non-planar programming.

A director program satisfying (C.2) is not, however, sufficient to ensure the existence of a continuous piecewise affine deformation $y$ satisfying (1.12) and (1.13). To illustrate this point, consider the design in Figure 11a. Here, we have a junction with three sectors of equal angle $2 \pi / 3$, and the director is programmed to bisect the sector angle (respectively, perpendicular to the bisector) on heating (respectively, cooling). This program satisfies the necessary condition (C.2). However in this case, due to the stretching part of the deformation upon actuation, the base of each triangle expands while the height contracts. Thus, it is clear geometrically that no series of rotations and/or translations of the three deformed triangles can bring about a continuous piecewise affine deformation of the entire junction. Conversely, if thermal actuation is reversed, as illustrated in Figure 11b with the color change of the director program, then the base of each triangle contracts and the height expands. In this case, a continuous piecewise affine deformation is realized by rotating each of the deformed triangles out-of-plane to form a 3-sided pyramid.

Figure 11b, by way of example, also highlights a simple scheme to form a compatible pyramidal junction. Indeed, if a junction has $K \geq 3$ sectors of equal angle $2 \pi / K$ as in Figure 12 , then programming the director to bisect this angle upon cooling (respectively, perpendicular to the bisector on heating) alway leads to a compatible $K$-sided pyramid. There are, of course, an infinite number of these types of junction, as emphasized with the designs in the right part of Figure 12. Most importantly though, these junctions can be used as unit cells to actuate more complex structures from nematic sheets. This is shown in Figure 13 with designs for actuating a box (a), rhombic triacontehedron (b) and azimuthally periodic structures (c). Each design incorporates a unit cell in Figure 12 as the building block.

The examples highlighted in Figure 13 illustrate that for even the simplest of building blocks, there is a richness of shape changing deformations of nematic elastomer sheets described by nonisometric origami. It should be noted, however, that these structure are in general degenerate. This is shown in Figure 13d where we design a program to actuate a rhombic dodecahedron upon cooling. Here though, we have done nothing to break the reflection symmetry associated with the building 

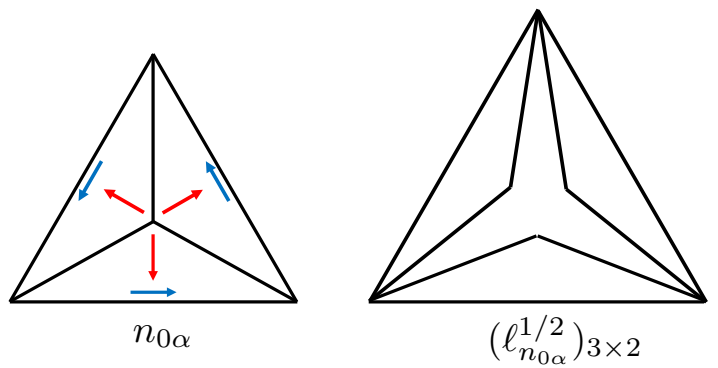

(a) Incompatible Junction
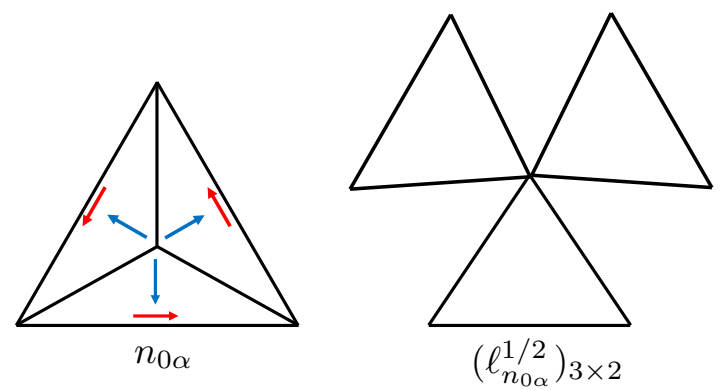

(b) Compatible Junction

Figure 11: Two junctions with director programs satisfying (C.2). Blue represents the design for cooling and red represents the design for heating. The stretch part of the deformation upon thermal actuation is plotted.

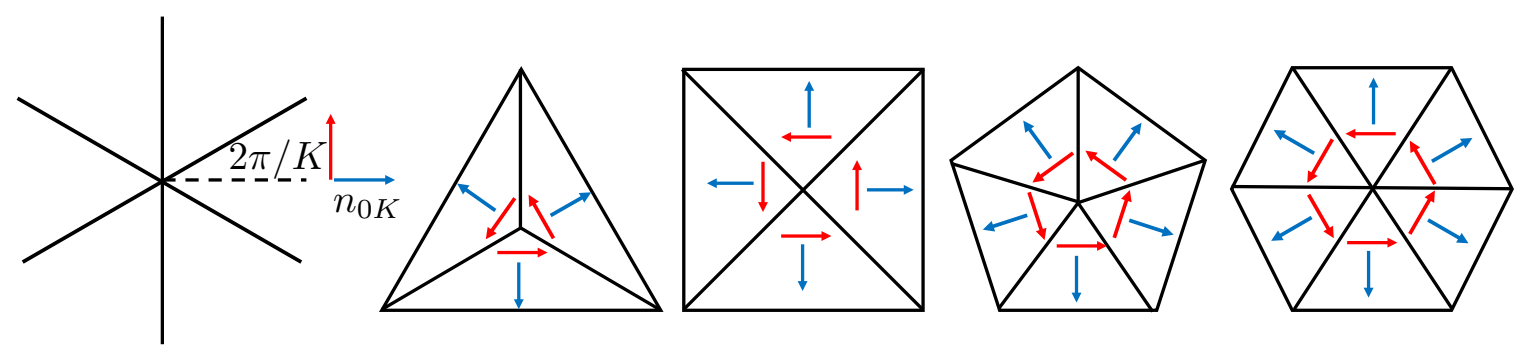

Figure 12: Simple scheme for compatible junction.

block. Thus, each interior junction is free to actuate either up or down. Therefore, in addition to possibly actuating the rhombic dodecahedron, the actuation of four alternative surfaces is a completely equivalent outcome given this framework. Such degeneracy was observed actuating conical defects by Ware et al. [41], where it was shown that each defect could actuate either up or down. However, it may be possible to suppress these degeneracies by introducing a slight bias in the thru thickness director orientation via twisted nematic prescription. This was intimated in another work by Fuchi et al. [25], where actuation of a box like structure was achieved through folds biased in the appropriate direction using such prescription. Thus, biasing would appear a promising means of breaking the reflection symmetry. Nevertheless, we do not address this here as it is difficult to analyze to the level of rigor intended for this work.

We now make one final comment on the design landscape for these constructions before proceeding to the discussion of our main result regarding the energy of nonisometric origami. Recall that the relations associated with (C.1) provide a complete, but not particularly transparent, description of nonisometric origami. Further, the more useful condition (C.2) is only necessary as we provided a counterexample to sufficiency in Figure 11a. In fact, to our knowledge, a complete characterization of the geometry of configurations satisfying (C.1) remains open. Nevertheless, we do expect an immense richness to such a characterization. For instance, in [38] a more general, but by no means complete, characterization of compatible three-faced junctions is worked out, and numerous non-trivial examples of compatibility emerge from the analysis. For these reasons, we feel a further pursuit in this direction appealing, though we do not probe deeper herein due to length considerations. 


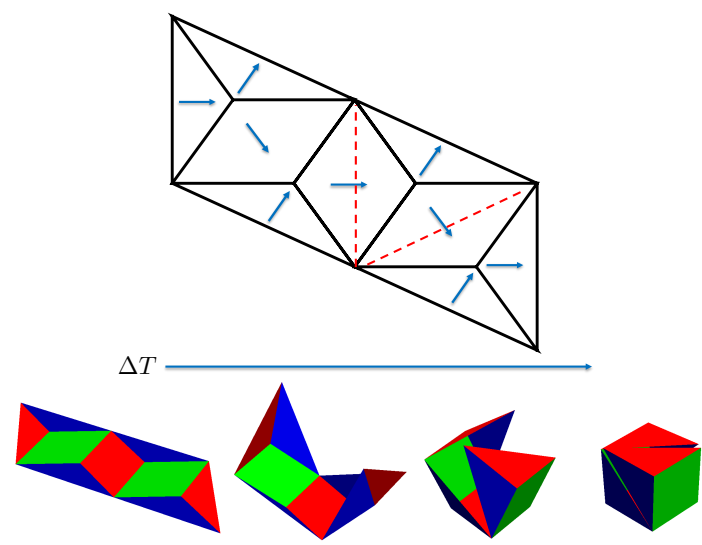

(a) Box construction

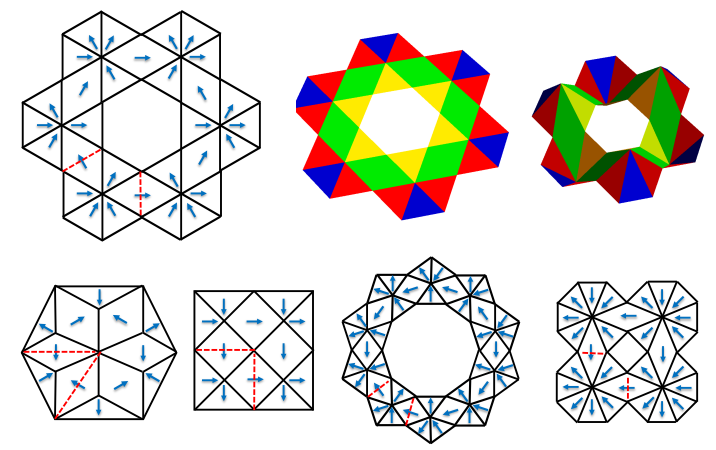

(c) Azimuthally Periodic Construction
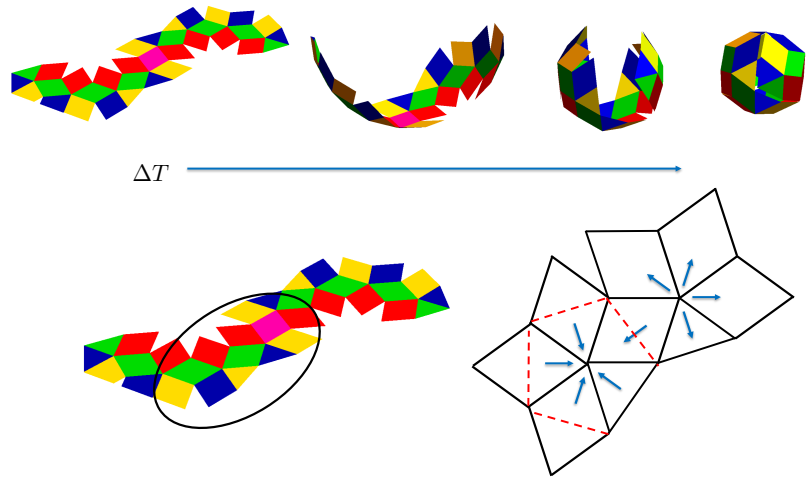

(b) Rhombic tricontahedron construction
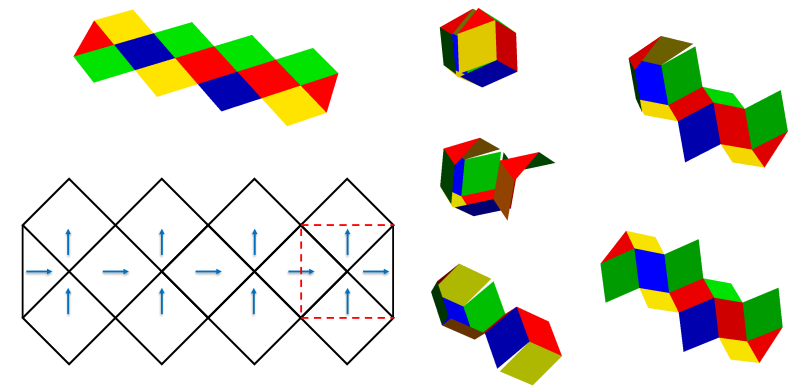

(d) Degeneracies

Figure 13: Examples of nonisometric origami. The design for cooling is shown. For heating, each director field is replaced by its respective perpendicular in the plane. The unit cells which form the building blocks for these constructions are highlighted in red.

\section{Proof of Proposition 2.3}

Recall that this calculation encapsulates an approximation to the boundary deformations $y \in$ $\left\{y_{b o x}, y_{r d}, y_{r t}\right\}$ given in $(2.23)$ of Section 2.3. We show this in three steps:

Step 1. There exists a $y_{1}^{\delta} \in C^{\infty}\left(\omega, \mathbb{R}^{3}\right)$ which approximates $y$ as in (2.25) and satisfies the derivative estimates (2.26) outside of the ball $B_{\delta / 2}(O)$.

We set $y_{0}^{\delta}: \omega \rightarrow \mathbb{R}^{3}$ as in Proposition 2.1 with $\rho_{0}^{\delta} \geq \delta / \sin \left(\theta_{K}\right)$ to be chosen later. In addition, we set $y_{c}: \omega \rightarrow \mathbb{R}^{3}$ as

$$
y_{c}(\tilde{x}):=\frac{r^{5 / 6}}{c_{K}^{r}} \rho\left(\cos \left(\frac{\tilde{\theta}_{K}}{\theta_{K}} \theta\right) e_{2}+\sin \left(\frac{\tilde{\theta}_{K}}{\theta_{K}} \theta\right) e_{2}\right)
$$

where $x_{1}=\rho \cos (\theta), x_{2}=\rho \sin (\theta)$ and $\tilde{\theta}_{K}, \theta_{K}$ defined in (2.23) and (2.24). This deformation is compared to $y$ in Figure 7 . The important features are that $y_{c}\left(x_{1}, 0\right)=y_{e_{1}}\left(x_{1}, 0\right)$ for $y_{e_{1}}$ in $(2.22)$ (due to the prefactor $r^{5 / 6} / c_{K}^{r}$ ) and the $\theta_{K}$ sector in Figure $4 \mathrm{~b}$ is deformed to $\tilde{\theta}_{K}$ for both $y_{c}$ and $y$. 
Hence, we define $y_{1}^{\delta}: \omega \rightarrow \mathbb{R}^{3}$ such that

$$
y_{1}^{\delta}=y_{0}^{\delta}+\psi_{\delta}\left(y_{c}-y_{0}^{\delta}\right)
$$

for $\psi_{\delta} \in C^{\infty}(\mathbb{R},[0,1])$ a smooth radial cutoff function $\psi_{\delta}=\psi_{\delta}(\rho)$ taking the value 1 in $B_{\rho_{0}^{\delta}}(O)$ and 0 outside $B_{\rho_{1}^{\delta}}(O)$ for some $\rho_{1}^{\delta}$ to be chosen subsequently.

We claim that after suitable choice of $\rho_{0}^{\delta} \rho_{1}^{\delta}$ and $\psi_{\delta}$, this deformation satisfies the desired properties for Step 1. First, we observe that $y_{1}^{\delta} \in C^{\infty}\left(\omega, \mathbb{R}^{3}\right)$ since $y_{c} \in C^{\infty}\left(\omega, \mathbb{R}^{3}\right)$ and $y_{0}^{\delta}$ is smooth outside of the ball $B_{\rho_{0}^{\delta}}(O)$. Note that it is not smooth up to the boundary since $\left|\tilde{\nabla} \tilde{\nabla} y_{c}\right| \rightarrow \infty$ as $\rho \rightarrow 0$. Next, we observe that $y_{1}^{\delta}$ satisfies $(2.25)$ and restricted to the set $\omega \backslash B_{\rho_{1}^{\delta}}(O)$, it satisfies (2.26). This follows from the fact that $y_{1}^{\delta}=y_{0}^{\delta}$ on $\omega \backslash B_{\rho_{1}^{\delta}}(O)$ and by Proposition 2.1, $y_{0}^{\delta}$ enjoys these properties. Next, by an identical argument to that of Proposition $2.2, y_{1}^{\delta}$ satisfies the upper bound estimates in (2.26) on the punctured disk $B_{\rho_{1}^{\delta}}(O) \backslash B_{\rho_{0}^{\delta}}(O)$. Finally, on the punctured disk $B_{\rho_{0}^{\delta}}(O) \backslash B_{\delta / 2}(O), y_{1}^{\delta}=y_{c}, y_{c}$ is independent of $\delta$ and $\tilde{\nabla} y_{c}$ is full-rank. So we conclude that is satisfies the estimates in (2.26) on this set.

Hence, it remains only to prove that $\left|\partial y_{1}^{\delta} \times \partial y_{1}^{\delta}\right| \geq c>0$ on the punctured disk $B_{\rho_{1}^{\delta}}(O) \backslash B_{\rho_{0}^{\delta}}(O)$ for $c$ depending only on $y$. This will follow after a suitable choice of $\rho_{0}^{\delta}$ and $\rho_{1}^{\delta}$, the result of an explicit calculation which we outline below.

We remark that we need only to show the result on the set $\omega_{e_{1}}$ in Figure $4 \mathrm{~b}$. This is due to the fact that $y_{1}^{\delta}$ enjoys the same underlying periodicity as $y$ and so each $\omega_{\tilde{R}_{K}^{\alpha} e_{1}}$ sector can be mapped back to $\omega_{e_{1}}$, deformed within this sector and subsequently rotated $Q_{K}^{\alpha}$ to achieve $y_{1}^{h}$ on $\omega_{\tilde{R}_{K}^{\alpha} e_{1}}$. The transformation rule is made explicit in (2.23). Further, it suffices to focus on the projection of $y_{1}^{\delta}$ onto the $\left\{e_{1}, e_{2}\right\}$ plane, denoted here as $\tilde{y}_{1}^{\delta}: \omega \rightarrow \mathbb{R}^{2}$, since $\left|\partial_{1} y_{1}^{\delta} \times \partial_{2} y_{1}^{\delta}\right| \geq\left|\operatorname{det}\left(\tilde{\nabla} \tilde{y}_{1}^{\delta}\right)\right|$.

We compute this planar gradient in the global Cartesian basis, and evaluate its determinant parameterized in polar coordinates so that

$$
\operatorname{det}\left(\tilde{\nabla} \tilde{y}_{1}^{\delta}\right)=\operatorname{det}\left(\partial_{1} \tilde{y}_{1}^{\delta} \otimes e_{1}+\partial_{2} \tilde{y}_{1}^{\delta} \otimes e_{2}\right)=: f_{K, \delta}^{r}(\rho, \theta) .
$$

Note, as suggested, $f_{K, \delta}^{r}$ depend on $r \in\left[1, r_{c}\right], \theta_{K}$ and $\delta$. We focus first on the region where $y_{0}^{\delta}$ is affine (i.e. $=\tilde{F}^{ \pm} \tilde{x}$ on $\omega_{e_{1}}$ in Figure $\left.4 \mathrm{~b}\right)$. This corresponds to $(\rho, \theta) \in\left(\omega_{e_{1}} \backslash \Gamma_{\delta}\right) \cap\left(B_{\rho_{1}^{\delta}}(O) \backslash B_{\rho_{0}^{\delta}}(O)\right)$. We find as the result of an explicit calculation that

$$
\begin{aligned}
f_{K, \delta}^{r}(\rho, \theta)= & \frac{r^{5 / 6}}{c_{K}^{r}}\left(A_{1, K}^{r}+A_{2, K}^{r}(\theta) \rho \psi_{\delta}^{\prime}(\rho)\right. \\
& \left.+\psi_{\delta}(\rho)\left(A_{3, K}^{r}(\theta)+A_{4, K}^{r}(\theta) \rho \psi_{\delta}^{\prime}(\rho)+A_{4, K}^{r}(\theta) \psi_{\delta}(\rho)\right)\right)
\end{aligned}
$$

in this regime. Here we denote the cases $K=3,4,5$ for the box, rhombic dodecahedron, and rhombic triacontahedron respectively. This is also the notation above in (2.23). In addition for $r \in\left(1, r_{c}\right]$, we find $A_{1, K}^{r}>0$ independent of $\theta$ as shown, $A_{3, K}^{r}(\theta)>0$ and $A_{2, K}^{r}(\theta), A_{4, K}(\theta) \geq 0$ with $A_{j, K}^{r}(\theta)=A_{j, K}^{r}(-\theta)$ for $\theta \in\left[-\theta_{K}, \theta_{K}\right]$ and for $j=2,3,4$. We also find for $\epsilon>0$ sufficiently small that

$$
\max _{K \in\{3,4,5\}} \max _{r \in\left(1, r_{c}\right]} \max _{\theta \in\left[0, \theta_{K}\right]}\left\{\frac{A_{2, K}^{r}(\theta)}{A_{1, K}^{r}}, \frac{A_{4, K}^{r}(\theta)}{A_{3, K}^{r}(\theta)}\right\} \leq \frac{3}{4}(1-\sqrt{\epsilon}) .
$$

This is shown in Figure 14. In addition, we choose our cutoff function $\psi_{\delta}$ such that

$$
\left\|\psi_{\delta}^{\prime}\right\|_{L^{\infty}} \leq \frac{1+\sqrt{\epsilon}}{\rho_{1}^{h}-\rho_{0}^{h}}
$$


for said $\epsilon$. This can always be done given the properties for $\psi_{h}$ below (D.2). Hence, since $\rho \psi_{h}^{\prime}(\rho) \leq 0$, we see given (D.4),

$$
\begin{aligned}
f_{K, \delta}^{r}(\rho, \theta) & \geq \frac{\bar{r}^{5 / 6}}{c_{K}^{r}}\left(A_{1, K}^{r}+\psi_{\delta}(\rho) A_{3, K}^{r}(\theta)\right)\left(1+\frac{3}{4}(1-\sqrt{\epsilon}) \rho \psi_{\delta}^{\prime}(\rho)\right) \\
& \geq C\left(1-\frac{3}{4}(1-\sqrt{\epsilon}) \rho_{1}^{\delta}\left\|\psi_{\delta}^{\prime}\right\|_{L^{\infty}}\right) \\
& \geq C\left(1-\frac{3}{4}(1-\epsilon) \frac{\rho_{1}}{\rho_{1}^{\delta}-\rho_{0}^{\delta}}\right) .
\end{aligned}
$$

Thus, requiring that

$$
\frac{\rho_{1}^{\delta}}{\rho_{1}^{\delta}-\rho_{0}^{\delta}} \leq \frac{4}{3}
$$

we satisfy $\operatorname{det} \tilde{\nabla} \tilde{y}_{1}^{\delta} \geq C \epsilon$ in this regime combining (D.6) with (D.3).

It remains to verify that $y_{1}^{\delta}$ is full-rank in the $\delta$-strip region in Figure $4 \mathrm{~b},(\rho, \theta) \in\left(\Gamma_{\delta} \cap \omega_{e_{1}}\right) \cap$ $\left(B_{\rho_{1}^{\delta}}(O) \backslash B_{\rho_{0}^{\delta}}(O)\right)$. Note, we are still free to choose $\rho_{0}^{\delta}$. Then $\rho_{1}^{\delta}$ will be set by the constraint (D.7). Therefore, to simplify the verification, we will choose $\rho_{0}^{\delta}=m \delta$ for a sufficiently large fixed constant $m>0$ such that in this regime $\theta \ll 1$. Now,

$$
\tilde{\nabla} \tilde{y}_{1}^{\delta}=\left(1-\psi_{\delta}\right) \tilde{\nabla} \tilde{y}_{c}+\psi_{\delta} \tilde{\nabla} \tilde{y}_{0}^{\delta}+\psi_{\delta}^{\prime}\left(\tilde{y}_{c}-\tilde{y}_{0}^{\delta}\right) \otimes e_{\rho}
$$

where $e_{\rho}=(\cos (\theta), \sin (\theta))$. With $\theta \ll 1$ due to our choice of $m$, it will become clear that the first two terms taken together have positive determinant and the last term is small and therefore does not effect the positivity of $\operatorname{det} \tilde{\nabla} \tilde{y}_{1}^{\delta}$. Indeed, we find

$$
\begin{aligned}
\left(1-\psi_{\delta}\right) \tilde{\nabla} \tilde{y}_{c}+\psi_{\delta} \tilde{\nabla} \tilde{y}_{0}^{\delta}= & \frac{r^{5 / 6}}{c_{K}^{r}}\left(\left(1-\psi_{\delta}\right)\left(\begin{array}{cc}
1 & 0 \\
0 & \frac{\tilde{\theta}_{K}}{\theta_{K}}
\end{array}\right)\right. \\
& \left.+\psi_{\delta}\left(\begin{array}{cc}
1 & \left(2 \lambda_{\delta}-1\right) \frac{(r-1)}{r \tan \left(\theta_{K}\right)} \\
0 & \frac{c_{K}^{r}}{r}
\end{array}\right)+O(\theta)\right) .
\end{aligned}
$$

This is the result of an explicit calculation using the formulas (2.7), (2.10), (2.22) and (D.1) under the assumption that $\theta \ll 1$. Hence, since $\tilde{\theta}_{K} / \theta_{K} \geq 1$ and $c_{K}^{r} \geq 1$ for $r \geq 1$, we find that

$$
\operatorname{det}\left(\left(1-\psi_{\delta}\right) \tilde{\nabla} \tilde{y}_{c}+\psi_{\delta} \tilde{\nabla} \tilde{y}_{0}^{\delta}\right) \geq \frac{r^{5 / 3}}{\left(c_{K}^{r}\right)^{2}}\left(\frac{1}{3}+O(\theta)\right) .
$$

Note, the $1 / 3$ term comes from setting $r=3$ as the worst case when the corners of the box merge. Now for the remaining term, with $\theta \ll 1$

$$
\begin{aligned}
\left|\psi_{\delta}^{\prime}\left(\tilde{y}_{c}-\tilde{y}_{0}^{\delta}\right) \otimes e_{\rho}\right| & =\left|\psi_{\delta}^{\prime}\left(\rho O\left(\theta^{2}\right)-\gamma_{\delta} \tilde{F}_{2 \times 2}^{-} e_{2}+\left(x_{2}-\gamma_{h}\right) \tilde{F}_{2 \times 2}^{+} e_{2}\right) \otimes\left(e_{1}+O(\theta)\right)\right| \\
& \leq\left|\psi_{\delta}^{\prime}\right|\left(\rho_{1}^{\delta}\left|O\left(\theta^{2}\right)\right|+\frac{\delta}{2}|O(1)|\right)(1+|O(\theta)|) \\
& \leq \frac{\rho_{1}^{\delta}}{\rho_{1}^{\delta}-\rho_{0}^{\delta}}\left|O\left(\theta^{2}\right)\right|+\frac{\delta}{\rho_{1}^{\delta}-\rho_{0}^{\delta}}|O(1)| \\
& \leq \frac{4}{3}\left|O\left(\theta^{2}\right)\right|+\frac{|O(1)|}{M-m}
\end{aligned}
$$



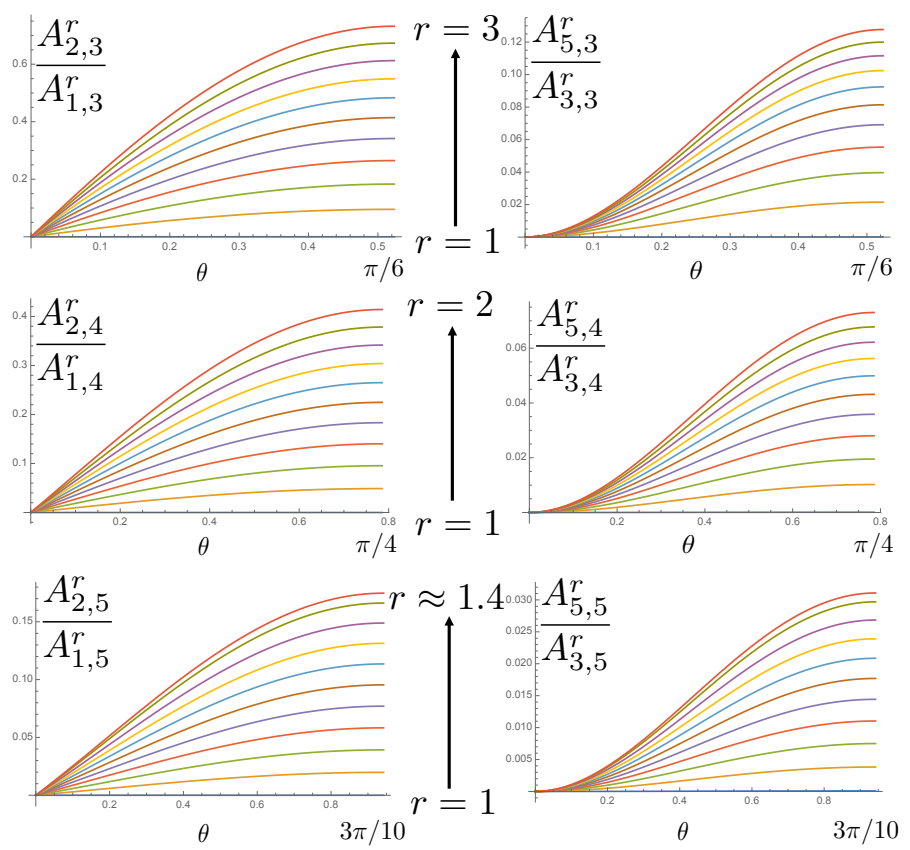

Figure 14: The coefficients associated with the maximization in (D.5) are plotted for the box (top), rhombic dodecahedron (middle) and rhombic triacontehedron (bottom). Multiple values for $r \in\left[1, r_{c}\right]$ are plotted, and we see monotonicity in the functions with respect to $r$. The worst case is given by the top-left graph. Thus, we see that in all cases, the range of these graphs is bounded from above by $3 / 4$ as asserted in (D.5).

where we set $\rho_{1}^{\delta}=M \delta$. Here, we again used (2.7) with $\tilde{F}_{2 \times 2}^{ \pm}$the $2 \times 2$ submatrix of $\tilde{F}^{ \pm}$given in (2.22) associated to the projection $\tilde{y}$. The estimate $4 / 3$ stems from the requirement (D.7). The important point here is that for any $\epsilon>0$, we may choose $m, M$ independent of $h$ and satisfying $M /(M-m) \leq 4 / 3$ such that $\left|\psi_{\delta}^{\prime}\left(\tilde{y}_{c}-\tilde{y}_{0}^{\delta}\right) \otimes e_{\rho}\right| \leq \epsilon$ since the $\left|O\left(\theta^{2}\right)\right|$ term in the estimate above is governed by the largeness of $m$. Thus, we can assure $\operatorname{det} \tilde{\nabla} \tilde{y}_{1}^{\delta}>0$ given (D.8) and an appropriate choice of $m$ and $M$. This completes the proof.

Step 2. There exists a $y_{2}^{\delta} \in C^{3}\left(\bar{\omega}, \mathbb{R}^{3}\right)$ which approximates $y$ in (2.25) and satisfies the upperbound derivative estimates in (2.26). Further, $\tilde{\nabla} y_{2}^{\delta}$ is full-rank everywhere but the origin.

For $y_{2}^{\delta}$, we modify $y_{1}^{\delta}$ in the ball $B_{\delta / 2}(O)$. Specifically, we replace $\rho$ in $y_{c}$ defined in (D.1) with

$$
f_{\delta}(\rho):= \begin{cases}\frac{160 \rho^{4}}{\delta^{3}}-\frac{720 \rho^{5}}{\delta^{4}}+\frac{1152 \rho^{6}}{\delta^{5}}-\frac{640 \rho^{7}}{\delta^{6}} & \text { if } \rho \in[0, \delta / 2) \\ \rho & \text { otherwise. }\end{cases}
$$

This choice is the result of a polynomial fit in which the function behaves like $\rho$ at $h / 2$, i.e. by setting $f_{\delta}(\delta / 2)=\delta / 2, f_{\delta}^{\prime}(\delta / 2)=1$ and $f_{\delta}^{\prime \prime}(\delta / 2), f_{\delta}^{(3)}(\delta / 2)=0$, and this fit ensures that the gradients satisfy

$$
\left|f_{\delta}^{\prime}\right| \leq C, \quad\left|f_{\delta}^{\prime \prime}\right| \leq C \delta^{-1}, \quad\left|f_{\delta}^{(3)}\right| \leq C \delta^{-2} \quad \text { on } B_{\delta / 2}(O)
$$

Hence, we let

$$
y_{2}^{\delta}=y_{0}^{\delta}+\psi_{\delta}\left(y_{c}^{\delta}-y_{0}^{\delta}\right)
$$


where $y_{c}^{\delta}$ is $y_{c}$ in (D.1) with this replacement and $\psi_{\delta}$ is the cutoff function of Step 1. Given that $f_{h}$ has the properties we have described, it follows that $\bar{y}_{2}^{\delta} \in C^{3}\left(\bar{\omega}, \mathbb{R}^{3}\right)$ and satisfies the upperbounds in (2.26) on this set. On the exceptional set, it satisfies both (2.25) and (2.26) since $y_{2}^{\delta}=y_{1}^{\delta}$ on this set, and we established this for $y_{1}^{\delta}$ in Step 1.

It remains to show that $y_{2}^{\delta}$ is full-rank everywhere but the origin. For this calculation, we need only consider this rank condition on $B_{\delta / 2}(O)$ where $y_{2}^{\delta}$ is modified from $y_{1}^{\delta}$. For this case, we find

$$
\left|\partial_{1} y_{2}^{\delta} \times \partial_{2} y_{2}^{\delta}\right|=\left|\partial_{1} y_{c}^{\delta} \times \partial_{2} y_{c}^{\delta}\right|=\frac{r^{5 / 3}}{\left(c_{K}^{r}\right)^{2}} \frac{\tilde{\theta}_{K}}{\theta_{K}}\left|\frac{f_{\delta}^{\prime} f_{\delta}}{\rho}\right|
$$

Hence noting that the quantity $\left|f_{\delta}^{\prime} f_{\delta}\right| / \rho$ does not vanish anywhere save the origin, the proof is complete.

Step 3. Proof of Proposition 2.3.

To satisfy the lower bound condition on the cross-product, we now replace $y_{c}^{\delta}$ in (D.10) with

$$
y_{c}^{\delta, \epsilon}=y_{c}^{\delta}+\epsilon\left(x_{1} e_{1}+x_{2} e_{3}\right) .
$$

We claim that with this replacement, the function $y_{\epsilon}^{\delta} \in C^{3}\left(\bar{\omega}, \mathbb{R}^{3}\right)$ given by

$$
y_{\epsilon}^{\delta}=y_{0}^{\delta}+\psi_{\delta}\left(y_{c}^{\delta, \epsilon}-y_{0}^{\delta}\right)
$$

has all the desired properties of Proposition 2.3 for $\epsilon>0$ chosen sufficiently small. Indeed, this perturbation does not modify $y_{2}^{\delta}$ outside $B_{\rho_{1}^{\delta}}(O)$ and so $(2.25)$ holds since it holds for $y_{2}^{\delta}$ in Step 2. In addition, the upperbound estimates in $(2.26)$ are unmodified by this perturbation. So we need only to show the lowerbound on the cross-product. This will be the case after an appropriate choice of $\epsilon$.

We are assured this lowerbound outside $B_{\rho_{1}^{\delta}}(O)$, so we need only consider two cases. First, on the set $B_{\rho_{1}^{\delta}}(O) \backslash B_{\delta / 2}(O)$, we find

$$
\tilde{\nabla} y_{\epsilon}^{\delta}=\tilde{\nabla} y_{1}^{\delta}+\tilde{\nabla}\left(\psi_{\delta} \epsilon\left(x_{1} e_{1}+x_{2} e_{3}\right)\right) \text {. }
$$

This last term is estimated as $\left|\tilde{\nabla}\left(\psi_{\delta} \epsilon\left(x_{1} e_{1}+x_{2} e_{3}\right)\right)\right| \leq C \epsilon$. Hence since $\left|\partial_{1} y_{1}^{\delta} \times \partial_{2} y_{2}^{\delta}\right| \geq c>0$ for $c$ depending only on $y$, and since $\tilde{\nabla} y_{\epsilon}^{\delta}$ is $\epsilon$ close to $\tilde{\nabla} y_{1}^{\delta}$ on this set, we conclude by continuity that there exists an $\bar{\epsilon}>0$ such that $\left|\partial_{1} y_{\epsilon}^{\delta} \times \partial_{2} y_{\epsilon}^{\delta}\right| \geq c / 2>0$ on $B_{\rho_{1}^{\delta}}(O) \backslash B_{\delta / 2}(O)$ for all $\epsilon \in(0, \bar{\epsilon})$.

Now, we set $\hat{\theta}_{K}=\tilde{\theta}_{K} / \theta_{K}$. Hence, in the ball $B_{h / 2}(O)$, we find explicitly that

$$
\begin{aligned}
\left(\partial_{1} y_{\epsilon}^{\delta} \times \partial_{2} y_{\epsilon}^{\delta}\right) \cdot e_{3} & =\frac{\hat{\theta}_{K} r^{5 / 3}}{\left(c_{K}^{r}\right)^{2}} \frac{f_{\delta}^{\prime} f_{h}}{\rho}+\epsilon g\left(\theta, f_{\delta}^{\prime}, f_{\delta} / \rho\right) \\
& =\bar{f}(\rho / \delta)+\epsilon \bar{g}(\theta, \rho / \delta)
\end{aligned}
$$

where $\bar{f}(\rho / \delta)$ in non-negative and vanishes only at $\rho / \delta=0$. Further, $\bar{g}$ is bounded. Thus, we choose an $\epsilon \in(0, \bar{\epsilon})$ such that this quantity is positive for all $\rho / \delta \geq c$ where we take $c \ll 1$. Note this $\epsilon$ only depends on $\rho / \delta$ and not on $\delta$ itself. Hence, with this $\epsilon$ we set $\tilde{\nabla} y_{\epsilon}^{\delta} \equiv \tilde{\nabla} y^{\delta}$, and note that this is full rank outside of $\rho / \delta \leq c \ll 1$.

Now in the set $\rho / \delta \leq c \ll 1$, we have the simplification

$$
f_{\delta} / \rho=160(\rho / \delta)^{3}+O\left((\rho / \delta)^{4}\right), \quad f_{\delta}^{\prime}=640(\rho / \delta)^{3}+O\left((\rho / \delta)^{4}\right)
$$


following the definition in (D.9). Using this simplification, we find that

$$
\begin{aligned}
& \left(\partial_{1} y^{\delta} \times \partial_{2} y^{\delta}\right) \cdot e_{1}=\epsilon\left(\frac{80 r^{5 / 6}}{c_{K}^{r}}\left(\frac{\rho}{\delta}\right)^{3} f_{1}\left(\hat{\theta}_{K}, \theta\right)+O\left((\rho / \delta)^{4}\right)\right) \text { for } \\
& f_{1}\left(\hat{\theta}_{K}, \theta\right):=\left(4-\hat{\theta}_{K}\right) \sin \left(\left(1+\hat{\theta}_{K}\right) \theta\right)+\left(4+\hat{\theta}_{K}\right) \sin \left(\left(\hat{\theta}_{K}-1\right) \theta\right) .
\end{aligned}
$$

Similarly, we find that

$$
\begin{aligned}
& \left(\partial_{1} y^{\delta} \times \partial_{2} y^{\delta}\right) \cdot e_{2}=-\epsilon^{2}-\epsilon\left(\frac{80 r^{5 / 6}}{c_{K}^{r}}\left(\frac{\rho}{\delta}\right)^{3} f_{2}\left(\hat{\theta}_{K}, \theta\right)+O\left((\rho / \delta)^{4}\right)\right) \quad \text { for } \\
& f_{2}\left(\hat{\theta}_{K}, \theta\right):=\left(4-\hat{\theta}_{K}\right) \cos \left(\left(1+\hat{\theta}_{K}\right) \theta\right)+\left(4+\hat{\theta}_{K}\right) \cos \left(\left(\hat{\theta}_{K}-1\right) \theta\right)
\end{aligned}
$$

We now verify that the quantities in (D.11) and (D.12) are never simultaneously 0. For this, we first note that in the calculations of interest $\hat{\theta}_{K} \in[1,2]$ (i.e. the box, rhombic dodecahedron and rhombic triacontehedron constructions in Figure 6). Hence, we find that

$$
f_{1}\left(\hat{\theta}_{K}, \theta\right)^{2}+f_{2}\left(\hat{\theta}_{K}, \theta\right)^{2}=2\left(17+\left(\hat{\theta}_{K}-1\right)\left(\hat{\theta}_{K}+1\right)-\left(\hat{\theta}_{K}-4\right)\left(\hat{\theta}_{K}+4\right) \cos (2 \theta)\right) \geq 4 \hat{\theta}_{K}^{2} .
$$

Now we suppose $\left(\partial_{1} y^{\delta} \times \partial_{2} y^{\delta}\right) \cdot e_{1}=0$. Then either $\rho=0$ which gives $\left(\partial_{1} y^{\delta} \times \partial_{2} y^{\delta}\right) \cdot e_{2}=-\epsilon^{2}$ or $f_{1}\left(\hat{\theta}_{K}, \theta\right)=O(\rho / h)$. Thus, the rank of $\tilde{\nabla} y^{\delta}$ vanishes on the set $\rho / \delta \leq c \ll 1$ only if $f_{1}\left(\hat{\theta}_{K}, \theta\right)=$ $O(\rho / \delta)$. But if this is the case, we also have

$$
f_{2}\left(\hat{\theta}_{K}, \theta\right) \geq 2 \hat{\theta}_{K}+O(\rho / \delta) \quad \text { or } \quad f_{2}\left(\hat{\theta}_{K}, \theta\right) \leq-2 \hat{\theta}_{K}+O(\rho / \delta)
$$

given the inequality in (D.14). In fact though, the latter inequality is impossible for the deformations of interest.

To see this, we first remark that the relevant sets to consider for these deformations are

$$
\begin{aligned}
& y \equiv y_{b o x}: \quad \theta \in[-\pi / 2, \pi / 2], \quad \hat{\theta}_{K} \in[1,2] ; \\
& y \equiv y_{r d}: \quad \theta \in[-\pi / 2, \pi], \quad \hat{\theta}_{K} \in[1,7 / 6] ; \\
& y \equiv y_{r t}: \quad \theta \in[-6 \pi / 10,6 \pi / 5], \quad \hat{\theta}_{K} \in[1,10 / 9] .
\end{aligned}
$$

This can be seen directly from Figure 6 , or alternatively it follows from the definitions of $\hat{\theta}_{K}$ and so $\tilde{\theta}_{K}$ in (2.24). Now, we assume $f_{2}$ in (D.13) satisfies the second inequality in (D.15). We will arrive at a contradiction given (D.16). Indeed, $\cos \left(\left(\hat{\theta}_{K}-1\right) \theta\right) \geq 0$ for these deformations. Thus by a crude estimate for $f_{2}$, we have for these deformations

$$
f_{2}\left(\hat{\theta}_{K}, \theta\right) \geq-4+\hat{\theta}_{K} .
$$

Hence, for the second inequality in (D.15), it must be that $\hat{\theta}_{K} \leq 4 / 3+O(\rho / \delta)$. Therefore, actually $\cos \left(\left(\hat{\theta}_{K}-1\right) \theta\right)$ is approximately bounded from below by $\cos (\pi / 6)=\sqrt{3} / 2$. Hence, in this regime, we have

$$
f_{2}\left(\hat{\theta}_{K}, \theta\right) \geq-4+\hat{\theta}_{K}+\frac{\sqrt{3}}{2}\left(4+\hat{\theta}_{K}\right)+O(\rho / \delta)>0 .
$$

This is the desired contradiction.

Finally, we can show that $\tilde{\nabla} y^{\delta}$ satisfies the cross product bound everywhere. Indeed, assume it does not. Then $f_{1}\left(\hat{\theta}_{K}, \theta\right)=O(\rho / \delta)$. This implies $f_{2}\left(\hat{\theta}_{K}, \theta\right) \geq 2 \hat{\theta}_{K}+O(\rho / \delta)$. Hence from (D.12) with $f_{2}$ positive, we conclude

$$
\left|\partial_{1} y^{\delta} \times \partial_{2} y^{\delta}\right| \geq \epsilon^{2}
$$

whenever $\left(\partial_{1} y^{\delta} \times \partial_{2} y^{\delta}\right) \cdot e_{1}=0$. This completes the proof of Proposition 2.3. 


\section{E Geometric rigidity and nematic elastomers}

First, we derive the key estimate which relates Geometric Rigidity [23] to the setting of nematic elastomers.

Proposition E.1. Let $\omega \subset \mathbb{R}^{3}$ bounded and Lipschitz. There exists a constant $C=C\left(r_{0}, r_{f}, \tau\right)$ with the following property: for all $h>0, Q_{\tilde{x}^{*}, h}:=(-h / 2, h / 2)^{3} \subset \Omega_{h}, W^{h} \in W^{1,2}\left(\Omega_{h}, \mathbb{R}^{3}\right)$, $N^{h} \in W^{1,2}\left(\Omega_{h}, \mathbb{S}^{2}\right)$ and $N_{0}^{h}$ as in $(1.7)$ with $n_{0} \in W^{1,2}\left(\omega, \mathbb{R}^{3}\right)$, there exists an associated constant rotation $R_{\tilde{x}^{*}}^{h} \in S O(3)$ such that

$$
\begin{aligned}
& \int_{Q_{\tilde{x}^{*}, h}}\left|\left(\ell_{N^{h}}^{f}\right)^{-1 / 2} \nabla Y^{h}\left(\ell_{N_{0}^{h}}^{0}\right)^{1 / 2}-R_{\tilde{x}^{*}}^{h}\right|^{2} d x \\
& \quad \leq C \int_{Q_{\tilde{x}^{*}, h}}\left(\operatorname{dist}^{2}\left(\left(\ell_{N^{h}}^{f}\right)^{-1 / 2} \nabla Y^{h}\left(\ell_{N_{0}^{h}}^{0}\right)^{1 / 2}, S O(3)\right)+h^{2}\left(\left|\nabla N^{h}\right|^{2}+\left|\tilde{\nabla} n_{0}\right|^{2}+1\right)\right) d x
\end{aligned}
$$

Proof. Let $Y^{h} \in W^{1,2}\left(\Omega_{h}, \mathbb{R}^{3}\right), N^{h} \in W^{1,2}\left(\Omega_{h}, \mathbb{S}^{2}\right)$ and $n_{0} \in W^{1,2}\left(\omega, \mathbb{S}^{2}\right)$ with $N_{0}^{h}$ as in (1.7). we fix $\tilde{x}^{*}$ such that $Q_{\tilde{x}^{*}, h} \subset \Omega_{h}$ and set

$$
A_{h}^{f}:=\frac{1}{\left|Q_{\tilde{x}^{*}, h}\right|} \int_{Q_{\tilde{x}^{*}, h}}\left(\ell_{N^{h}}^{f}\right)^{1 / 2} d x, \quad A_{h}^{0}:=\frac{1}{\left|Q_{\tilde{x}^{*}, h}\right|} \int_{Q_{\tilde{x}^{*}, h}}\left(\ell_{n_{0}}^{0}\right)^{-1 / 2} d x .
$$

Because of the structure of the step-length tensors, these averages are positive definite, and each of the eigenvalues lives in a compact set of the positive real numbers depending only on $r_{f}$ and $r_{0}$ (in particular, this set does not depend on $h$ ). Hence, these linear maps belong to a family of $h$-indepdent Bilipschtiz maps with controlled Lipschitz constant, and so we write $A^{f} \equiv A_{h}^{f}$ and $A^{0} \equiv A_{h}^{0}$ in sequel.

Now, we set

$$
V^{h}(s)=\left(A^{f}\right)^{-1} Y^{h}\left(\left(A^{0}\right)^{-1} s\right), \quad s \in\left(A^{0}\right) Q_{\tilde{x}^{*}, h} .
$$

We observe that $V^{h} \in W^{1,2}\left(\left(A^{0}\right) Q_{\tilde{x}, h}, \mathbb{R}^{3}\right)$ by the regularity of $Y^{h}$. Therefore by Geometric Rigidity [23], there exists a constant rotation $R_{\tilde{x}}^{h} \in S O(3)$ such that

$$
\begin{aligned}
\int_{Q_{\tilde{x}^{*}, h}}\left|\left(A^{f}\right)^{-1} \nabla Y^{h}(x)\left(A^{0}\right)^{-1}-R_{\tilde{x}^{*}}^{h}\right|^{2} d x=\left|\operatorname{det} A^{0}\right|^{-1} \int_{\left(A^{0}\right) Q_{\tilde{x}^{*}, h}}\left|\nabla V^{h}(s)-R_{\tilde{x}^{*}}^{h}\right|^{2} d s \\
\leq\left|\operatorname{det} A^{0}\right|^{-1} C\left(\left(A^{0}\right) Q_{\tilde{x}^{*}, h}\right) \int_{\left(A_{\omega}^{0}\right) Q_{\tilde{x}^{*}, h}} \operatorname{dist}^{2}\left(\nabla V^{h}(s), S O(3)\right) d s \\
\quad=C\left(\left(A^{0}\right) Q_{\tilde{x}^{*}, h}\right) \int_{Q_{\tilde{x}^{*}, h}} \operatorname{dist}^{2}\left(\left(A^{f}\right)^{-1} \nabla Y^{h}(x)\left(A^{0}\right)^{-1}, S O(3)\right) d x
\end{aligned}
$$

The constant $C\left(\left(A^{0}\right) Q_{\tilde{x}^{*}, h}\right)$ can be chosen uniformly for a family of domains which are Bilipschitz equivalent with controlled Lipschitz constant. Hence, actually we can choose $C\left(r_{0}, Q_{\tilde{x}^{*}, h}\right) \geq$ $C\left(\left(A^{0}\right) Q_{\tilde{x}^{*}, h}\right)$. Moreover, the constant is invariant under translation and dilatation. Hence, actually we have $C\left(r_{0}, Q_{\tilde{x}^{*}, h}\right)=C\left(r_{0}\right)$ for any $Q_{\tilde{x}^{*}, h} \subset \Omega_{h}$. These properties are given in Friesecke, James and Müller, Theorem 9 [24]. Since $r_{0}$ is fixed in this calculation, we write $C\left(r_{0}\right) \equiv C$, and thus

$$
\int_{Q_{\tilde{x}^{*}, h}}\left|\left(A^{f}\right)^{-1} \nabla Y^{h}(x)\left(A^{0}\right)^{-1}-R_{\tilde{x}^{*}}^{h}\right|^{2} d x \leq C \int_{Q_{\tilde{x}^{*}, h}} \operatorname{dist}^{2}\left(\left(A^{f}\right)^{-1} \nabla Y^{h}(x)\left(A^{0}\right)^{-1}, S O(3)\right) d x
$$

from (E.2). 
Since we will no longer be dealing with a change of variables in this proof, we now drop the explicit dependence on $x$ inside the integrals. We observe by the key estimate (E.3) that

$$
\begin{aligned}
& \int_{Q_{\tilde{x}^{*}, h}}\left|\nabla Y^{h}-\left(A^{f}\right) R_{\tilde{x}^{*}}^{h}\left(A^{0}\right)\right|^{2} d x \leq C \int_{Q_{\tilde{x}^{*}, h}}\left|\left(A^{f}\right)^{-1} \nabla Y^{h}\left(A^{0}\right)^{-1}-R_{\tilde{x}^{*}}^{h}\right|^{2} d x \\
& \leq \leq \int_{Q_{\tilde{x}^{*}, h}} \operatorname{dist}^{2}\left(\left(A^{f}\right)^{-1} \nabla Y^{h}\left(A^{0}\right)^{-1}, S O(3)\right) d x \leq C \int_{Q_{\tilde{x}^{*}, h}} \operatorname{dist}^{2}\left(\nabla Y^{h},\left(A^{f}\right) S O(3)\left(A^{0}\right)\right) d x \\
& \leq C \int_{Q_{\tilde{x}^{*}, h}}\left(\operatorname{dist}^{2}\left(\nabla Y^{h},\left(\ell_{N^{h}}^{f}\right)^{1 / 2} S O(3)\left(\ell_{N_{0}^{h}}^{0}\right)^{-1 / 2}\right)+\left|\left(\ell_{N^{h}}^{f}\right)^{1 / 2}-A^{f}\right|^{2}+\left|\left(\ell_{N_{0}^{h}}^{0}\right)^{-1 / 2}-A^{0}\right|^{2}\right) d x \\
& \leq C \int_{Q_{\tilde{x}^{*}, h}}\left(\operatorname{dist}^{2}\left(\left(\ell_{N^{h}}^{f}\right)^{-1 / 2} \nabla Y^{h}\left(\ell_{N_{0}^{h}}^{0}\right)^{1 / 2}, S O(3)\right)+h^{2}\left|\nabla N^{h}\right|^{2}\right. \\
&\left.\quad+\left|\left(\ell_{n_{0}}^{0}\right)^{-1 / 2}-A^{0}\right|^{2}+\left|\left(\ell_{N_{0}^{h}}^{0}\right)^{-1 / 2}-\left(\ell_{n_{0}}^{0}\right)^{-1 / 2}\right|^{2}\right) d x \\
& \leq C \int_{Q_{\tilde{x}^{*}, h}}\left(\operatorname{dist}^{2}\left(\left(\ell_{N^{h}}^{f}\right)^{-1 / 2} \nabla Y^{h}\left(\ell_{N_{0}^{h}}^{0}\right)^{1 / 2}, S O(3)\right)+h^{2}\left(\left|\nabla N^{h}\right|^{2}+\left|\tilde{\nabla} n_{0}\right|^{2}+1\right)\right) d x
\end{aligned}
$$

Here, the constant $C=C\left(r_{0}, r_{f}, \tau\right)$ is due to several applications of the triangle inequality and the fact that the norm of the step-length tensors, inverses and averages are compact and this depends only on $r_{f}, r_{0}$. We have also applied the standard Poincaré inequality given the averages (E.1), and used that the diameter of $Q_{\tilde{x}^{*}, h}$ is $h$ and that the gradients of the step-length tensors are controlled by the gradients of the directors. Finally, from the assumed control of non-idealities for $N_{0}^{h}$ in (1.7), we have the estimate $\left\|\left(\ell_{N_{0}^{h}}^{0}\right)^{-1 / 2}-\left(\ell_{n_{0}}^{0}\right)^{-1 / 2}\right\|_{L^{\infty}} \leq c\left(r_{0}\right) \tau h$. This gives the dependence on $\tau$ in the constant.

Now using (E.4), we find that

$$
\begin{aligned}
\int_{Q_{\tilde{x}^{*}, h}} & \left|\left(\ell_{N^{h}}^{f}\right)^{-1 / 2} \nabla Y^{h}\left(\ell_{N_{0}^{h}}^{0}\right)^{1 / 2}-R_{\tilde{x}^{*}}^{h}\right|^{2} d x \leq C \int_{Q_{\tilde{x}^{*}, h}}\left|\nabla Y^{h}-\left(\ell_{N^{h}}^{f}\right)^{1 / 2} R_{\tilde{x}^{*}}^{h}\left(\ell_{N_{0}^{h}}^{0}\right)^{-1 / 2}\right|^{2} d x \\
& \leq C \int_{Q_{\tilde{x}^{*}, h}}\left(\left|\nabla Y^{h}-\left(\ell_{N^{h}}^{f}\right)^{1 / 2} R_{\tilde{x}^{*}}^{h}\left(\ell_{n_{0}}^{0}\right)^{-1 / 2}\right|^{2}+\left|\left(\ell_{N_{0}^{h}}^{0}\right)^{-1 / 2}-\left(\ell_{n_{0}}^{0}\right)^{-1 / 2}\right|^{2}\right) d x \\
& \leq C \int_{Q_{\tilde{x}^{*}, h}}\left(\left|\nabla Y^{h}-\left(A^{f}\right) R_{\tilde{x}^{*}}^{h}\left(A^{0}\right)\right|^{2}+h^{2}+\left|\left(\ell_{N^{h}}^{f}\right)^{1 / 2}-A^{f}\right|^{2}+\left|\left(\ell_{n_{0}}^{0}\right)^{-1 / 2}-A^{0}\right|^{2}\right) d x \\
& \leq C \int_{Q_{\tilde{x}^{*}, h}}\left(\operatorname{dist}^{2}\left(\left(\ell_{N^{h}}^{f}\right)^{-1 / 2} \nabla Y^{h}\left(\ell_{n_{0}}^{0}\right)^{1 / 2}, S O(3)\right)+h^{2}\left(\left|\nabla N^{h}\right|^{2}+\left|\tilde{\nabla} n_{0}\right|^{2}+1\right)\right) d x
\end{aligned}
$$

as desired.

Now we note that the approximations in Lemma 5.1 are not new. They essentially follow from the same argument as that of Theorem 10 in Friesecke, James and Müller [24], modified appropriately for nematic elastomers using the estimate in Proposition E.1. In the general context of non-Euclidean plates, there is a recent body of literature on such estimates (e.g. Lewicka and Pakzad (Lemma 4.1) [30] and Lewicka et al. (Theorem 1.6) [29], (Lemma 2.3) [10]). Thus briefly:

Proof of Lemma 5.1. We repeat steps 1-3 in the proof of Theorem 10 in [24] with some modification due to our nematic elastomer setting. The lemma follows by the estimate in Proposition E.1. 


\section{References}

[1] H. Aharoni, E. Sharon, and R. Kupferman. Geometry of Thin Nematic Elastomer Sheets. Phys Rev Lett, 113(25):257801, Dec. 2014.

[2] J. M. Ball and R. D. James. Fine phase mixtures as minimizers of energy. In Analysis and Continuum Mechanics, pages 647-686. Springer, 1989.

[3] M. Barchiesi and A. DeSimone. Frank energy for nematic elastomers: a nonlinear model. ESAIM: Control, Optimisation and Calculus of Variations, 21(2):372-377, 2015.

[4] M. Barchiesi, D. Henao, and C. Mora-Corral. Local invertibility in sobolev spaces with applications to nematic elastomers and magnetoelasticity. 2015.

[5] H. B. Belgacem. Une méthode de $\gamma$-convergence pour un modele de membrane non linéaire. Comptes Rendus de l'Académie des Sciences-Series I-Mathematics, 324(7):845-849, 1997.

[6] P. Bella and R. V. Kohn. Metric-induced wrinkling of a thin elastic sheet. Journal of Nonlinear Science, 24(6):1147-1176, 2014.

[7] P. Bella and R. V. Kohn. Wrinkles as the result of compressive stresses in an annular thin film. Communications on Pure and Applied Mathematics, 67(5):693-747, 2014.

[8] K. Bhattacharya. Microstructure of martensite: why it forms and how it gives rise to the shape-memory effect, volume 2. Oxford University Press, 2003.

[9] K. Bhattacharya and R. D. James. A theory of thin films of martensitic materials withapplications to microactuators. Journal of the Mechanics and Physics of Solids, 47(3):531-576, 1999.

[10] K. Bhattacharya, M. Lewicka, and M. Schäffner. Plates with incompatible prestrain. Archive for Rational Mechanics and Analysis, pages 1-39, 2015.

[11] P. Bladon, E. Terentjev, and M. Warner. Transitions and instabilities in liquid crystal elastomers. Physical Review E, 47(6):R3838, 1993.

[12] J. A. Blume. Compatibility conditions for a left cauchy-green strain field. Journal of elasticity, 21(3):271-308, 1989.

[13] P. Cesana, P. Plucinsky, and K. Bhattacharya. Effective behavior of nematic elastomer membranes. Archive for Rational Mechanics and Analysis, 218(2):863-905, 2015.

[14] S. Conti, A. DeSimone, and G. Dolzmann. Semisoft elasticity and director reorientation in stretched sheets of nematic elastomers. Physical Review E, 66(6):061710, 2002.

[15] S. Conti, A. DeSimone, and G. Dolzmann. Soft elastic response of stretched sheets of nematic elastomers: a numerical study. Journal of the Mechanics and Physics of Solids, 50(7):14311451, 2002.

[16] S. Conti and G. Dolzmann. Derivation of elastic theories for thin sheets and the constraint of incompressibility. In Analysis, modeling and simulation of multiscale problems, pages 225-247. Springer, 2006. 
[17] S. Conti and G. Dolzmann. $\Gamma$-convergence for incompressible elastic plates. Calculus of Variations and Partial Differential Equations, 34:531-551, 2009.

[18] S. Conti and F. Maggi. Confining thin elastic sheets and folding paper. Archive for Rational Mechanics and Analysis, 187:1-48, 2008.

[19] B. Dacorogna. Direct methods in the calculus of variations, volume 78. Springer Science \& Business Media, 2007.

[20] P. G. de Gennes and J. Prost. The physics of liquid crystals. Number 83. Oxford university press, 1995.

[21] A. DeSimone and G. Dolzmann. Macroscopic response of nematic elastomers via relaxation of a class of so (3)-invariant energies. Archive for rational mechanics and analysis, 161(3):181-204, 2002 .

[22] E. Efrati, E. Sharon, and R. Kupferman. The metric description of elasticity in residually stressed soft materials. Soft Matter, 9(34):8187-8197, 2013.

[23] G. Friesecke, R. D. James, and S. Müller. A theorem on geometric rigidity and the derivation of nonlinear plate theory from three-dimensional elasticity. Communications on Pure and Applied Mathematics, 55:1461-1506, 2002.

[24] G. Friesecke, R. D. James, and S. Müller. A hierarchy of plate models derived from nonlinear elasticity by gamma-convergence. Archive for rational mechanics and analysis, 180(2):183-236, 2006.

[25] K. Fuchi, T. H. Ware, P. R. Buskohl, G. W. Reich, R. A. Vaia, T. J. White, and J. J. Joo. Topology optimization for the design of folding liquid crystal elastomer actuators. Soft matter, 11(37):7288-7295, 2015.

[26] P. Hornung. Approximation of flat w 2, 2 isometric immersions by smooth ones. Archive for rational mechanics and analysis, 199(3):1015-1067, 2011.

[27] R. V. Kohn and S. Müller. Surface energy and microstructure in coherent phase transitions. Communications on Pure and Applied Mathematics, 47(4):405-435, 1994.

[28] I. Kundler and H. Finkelmann. Strain-induced director reorientation in nematic liquid single crystal elastomers. Macromolecular rapid communications, 16(9):679-686, 1995.

[29] M. Lewicka, L. Mahadevan, and M. R. Pakzad. The föppl-von kármán equations for plates with incompatible strains. In Proceedings of the Royal Society of London A: Mathematical, Physical and Engineering Sciences, volume 467, pages 402-426. The Royal Society, 2011.

[30] M. Lewicka and M. R. Pakzad. Scaling laws for non-euclidean plates and the w 2, 2 isometric immersions of riemannian metrics. ESAIM: Control, Optimisation and Calculus of Variations, 17(04):1158-1173, 2011.

[31] C. Modes, K. Bhattacharya, and M. Warner. Gaussian curvature from flat elastica sheets. In Proceedings of the Royal Society of London A: Mathematical, Physical and Engineering Sciences, volume 467, pages 1121-1140. The Royal Society, 2011. 
[32] C. D. Modes and M. Warner. Blueprinting nematic glass: Systematically constructing and combining active points of curvature for emergent morphology. Physical Review E, 84(2):021711, 2011.

[33] C. D. Modes and M. Warner. Shape-programmable materials. Physics Today, 69(1):32-38, Jan. 2016.

[34] L. Modica. The gradient theory of phase transitions and the minimal interface criterion. Archive for Rational Mechanics and Analysis, 98(2):123-142, 1987.

[35] L. Modica and S. Mortola. Un esempio di $\gamma$-convergenza. Boll. Un. Mat. Ital. B (5), 14(1):285299, 1977.

[36] C. Mostajeran. Curvature generation in nematic surfaces. Physical Review E, 91(6):062405, 2015.

[37] M. R. Pakzad et al. On the sobolev space of isometric immersions. Journal of Differential Geometry, 66(1):47-69, 2004.

[38] P. Plucinsky. Deformation of nematic elastomer sheets. PhD thesis, California Institute of Technology, In preparation.

[39] P. Plucinsky, M. Lemm, and K. Bhattacharya. Programming complex shapes in thin nematic elastomer and glass sheets. Phys. Rev. E, 94:010701, Jul 2016.

[40] K. Trabelsi. Modeling of a membrane for nonlinearly elastic incompressible materials via gamma-convergence. Analysis and Applications, 4(01):31-60, 2006.

[41] T. H. Ware, M. E. McConney, J. J. Wie, V. P. Tondiglia, and T. J. White. Voxelated liquid crystal elastomers. Science, 347(6225):982-984, 2015.

[42] M. Warner, C. Modes, and D. Corbett. Curvature in nematic elastica responding to light and heat. In Proceedings of the Royal Society of London A: Mathematical, Physical and Engineering Sciences, volume 466, pages 2975-2989. The Royal Society, 2010.

[43] M. Warner and E. M. Terentjev. Liquid crystal elastomers, volume 120. OUP Oxford, 2003. 